HEREDTTX AND EVOLUTION

BIFE ANO DEATH

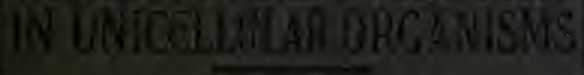

\title{
it S.DENTINTS
}

is

1
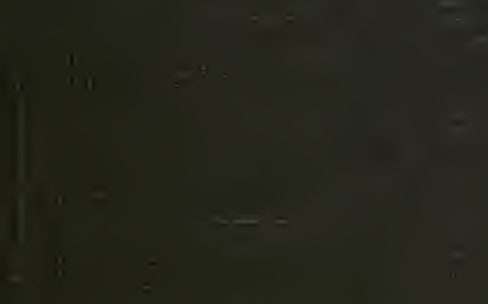

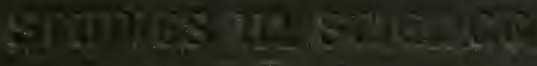




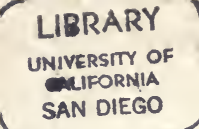


18.8.gemsings 



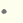




\title{
LIFE AND DEATH HEREDITY AND EVOLUTION IN UNICELLULAR ORGANISMS
}

\author{
LECTURES DELIVERED \\ UNDER THE
}

RICHARD B. WESTBROOK FREE LECTURESHIP FOUNDATION

AT THE

WAGNER FREE INSTITUTE OF SCIENCE

PHILADELPHIA 


\section{BA D GER'S STUDIES IN SCIENCE}

The Higher Usefulness of Sctence. By William Emerson Ritter.

The Unity of the Organism, or The Organismal Conception of Life. Two volumes. E'Iustrated. By William Emerson Ritter.

The Beginnings of Science. By Edward J. Menge.

The Probable Infinity of Nature AND LifE. By William Emerson Ritter. an Organsmal Conception of ConscIOUSNEss. By William Emerson Ritter. INSECT BeHavior. Illustrated. By Paul G. Howes.

Life and Death, Heredity and EvoluTION IN UNICELLULAR ORgaNisMS. Illustrated. By H.S. Jennings.

The Orntrhology of Chester County, Pennsyluania. Illustrated. By Frank L. Burns.

Familiar Studies of Wild Birds. Illustrated. By F.N.Whitman.

Questions and Outlines in General Chemistry. By W. S. Haldeman.

Santary Entomology. By W. Dwight Pierce.

RICHARD G. BADGER, PUBLISHER, BOSTON 


\title{
LIFE AND DEATH HEREDITY AND EVOLUTION IN UNICELLULAR ORGANISMS
}

\author{
BY \\ H. S. JENNINGS
}

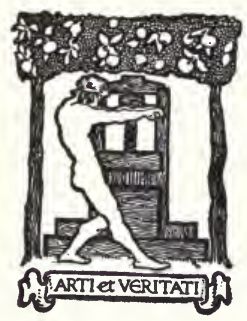

BOSTON

RICHARD G. BADGER

THE GORHAM PRESS 
Copyright, 1920, BY H. S. Jenninga

All Rights Reserved

Made in the United States of America

The Gorham Press, Boston, U. S. A. 


\section{PREFACE}

7 HE present work is not a book on Protozoology, but on 1 Genetics, employing Protozoa as material, and comparing the conditions there found with those in higher organisms.

To select from a great mass of varied material, not yet reduced by science to unity, those features that appear most significant for certain general questions is a task of difficulty, not unattended with the danger of justifying the critic. I cannot hope to entirely escape that danger. Much that is of extreme interest must be omitted, if any clearness of outline is to be preserved. A certain onesidedness appears inevitable, unless an encyclopedic work is attempted. The relatively great prominence given to the infusoria in these lectures is an example; the heterogeneous and still more imperfectly known genetic phenomena in the other Protozoa lend themselves less readily to a unified presentation. I can only hope that the limitations of the work aid in defining certain large problems.

Technical terms have been avoided. This is not alone because the lectures were for an audience not composed of specialists. Technical terms, in spite of their convenience, bring many disadvantages, even in strict scientific work. They seem to give to phenomena a distinctness and uniqueness which does not exist in nature. They create separate entities for things that are mere variations on a general theme. Any phenomenon has many-sided relations to the others; to bring these out we have not hesitated even 
to employ in different passages diverse designations for the same thing. What we observe in the Protozoa are combinations of chemicals; of matter and energy, with their characteristic activities. Technical terms tend to set these apart - and render them unintelligible; what we need is to render them intelligible by showing their community with the world of every-day experience.

The book deals with heredity, variation, evolution, as present physiology, not as past history. It discusses what now occurs, not what may have occurred in the past. Hence discussion of the origin of the conditions now existing will hardly be found.

\section{Acknowledgments}

To the Editor and Publishers of Genetics I am indebted for the use of the blocks for Figures 12 and 23, taken from Genetics, volume 1. To my wife, Louise Burridge Jennings, I am indebted for the drawing of the remainder of the figures in the book.

Johns Hopkins University,

Baltimore, $M d$. 


\section{CONTENTS}

I. General Survey of the Life History in the Protozos, with the Questions it Raises. Life, Death, Reproduction, Mating, Rejuvenescence, "Potential Immortality" . . .

II. Heredity and Variation in Protozoa, in Reproddction from a Single Parent. "The Inheritance of Acquired Charac" ters." The Existence of Many Diverse Stocks in a Single Species. Constancy of the Stocks. "Pure Line Inheritance" and the Results of Selection. Difficulties for the Theort of Evolution . . . . . . . . . . . .

III. Results of Intense and Long Continued Study of Changes in a Stock. Inherited Variations in the Pure Race. Visible Evolution . . . . . . . . . . . . . .

IV. Can We Experimentally Change the Hereditary Characters? Heredity of Environmental EFfects. Heredity aNd Variation in Bacteria and Similar Organisms . . . .

V. The Natural History of Mating. Sex, Itg Nature and Consequences. Sex in the Protozoa. Is Sex Coextensive with Life and Necessart to its Continuance? . . . . . .

VI. What Are the Results of Mating? Rejuvenescence and Mating. Heredity and Variation, and Mating . . .

VII. How Does Mating Bring About Both Biparental Inheritance, and Diversity in Hereditary Characters? What Effect Has Mating on the Stock as a Whole? Does It Increase Variation? Does It Decrease Variation? What Is Its Relation to Evolution? . . . . . . . . . 170

VIII. Comparison of the Genetic Phenomena in the Protozon with Those in Higher Organisms. General View of Development, Mating and Evolution • . . . . . . . . 


\section{LIST OF ILLUSTRATIONS}

FIGURE

PAGE

1. Stmpler Forms of Protista . . . . . . . . . . . 15

2. Some of the More Complex Protozoa . . . . . . . 16

3. Strdcture of One of the Most Complex of the Protozon . 17

4. Division of an Infusorian, Paramecium . . . . . . . 20

5. Diagram of the Descent of Generations in Protozoa - . 21

6. Conjugation of Paramecium . . . . . . . . . . . 22

7. Diagram of the Process of Division in Paramecium . . . 25

8. Diagram of the Internal Processes in the Conjugation of Paramecium Caddatum . . . . . . . . . . . . 26

9. Diagram Showing What Occurs in Endomixis . . . . . 31

10. Reproduction in Amgeba . . . . . . . . . . . . . . 39

11. Reproduction in Difflugia . . . . . . . . . . . 40

12. Two Parents, with Their Offspring, Just Before Separatiox in Difflugia Corona . . . . . . . . . . . . . 41

13. Reproduction in Stylonychia . . . . . . . . . . 43

14. Inheritance from Mutilated Parents, in Difflugia Corona 45

15. Reprontction in an Infegorian (Paramecium) in Which the Anterior End Has Been Cut OfF . . . . . . . . . 46

16. Reproduction for Several Generations of a Paramectum . 47

17. Reproduction in a Paramecium Bearing a Small Abnormal Projection Near the Posterior End . . . . . . . . 47

18. Reproduction in a Deformed Individual of Parameciom . 48

19. Difflugia Corona . . . . . . . . . . . . . . 51

20a. Difflugla Corona . . . . . . . . . . . . 52

20b. Difflugia Corona . . . . . . . . . . . . . 53

21. Difflugia Corona; Portions of Fodr Families . . . . . 55

22. Eight Diverse Families of Parameciom, Showing Variations 57

23. Difflugia Corona, to Show the Characters Studied in the New Work on Inheritance . . . . . . . . . . 71 
24a. Parts of Five Hereditarily Diverse Branches of a Single Family in Difflugia Corona . . . . . . . . . .

24b. Parts of Five Hereditarily Diverse Branches of a Single Family in Difflugia Corona

25. The Infusorian Strlonychia

26. Arcella Vulgaris . . . . . . . . . . . . 83

27. Trypanosoma Brucer . . . . . . . . . . . . . 97

28. Organisms Used in Dalinger's Experiments on the Effects of High Temperatures

29. Chromosomes and Their Mating

30. Diagram Showing the Chief Processes in the Conjugation of Paramecium Caudatum

31. Differences Between the Chromosomes of the Nuclei in the Two Sexes

32. The Active and Passive Portions of Cell and Nucleug in the EgG of the Whitefish

33. Beginning of Conjugation in Epistylis

34. Successive Steps in the Process of Conjugation in Vorticella Nebulifera

35. Diagrams of the Microndclear Processes in Conjegation, in Paramecium and Vorticella . . . . . . . . . . . 122

36. Mating in the Mould, Mucor . . . . . . . . . 123

37. Mating of Similar Celis, from the Alga Stephanosphera * 126

38. ChILodon . . . . . . . . . . . . . . . 129

39. Conjugation of Chilodon . . • • • • • • • . . 129

40. The Exchange of the Half Nuclei in Paramecium Caudatum 131

41. Mating of Two Halves of the Nucleus of Same Cell . 134

42. Two Methods of Conjugation in Spirogrra . . . . . 135

43. Process of Conjugation of Two Branches of the Same Plant. 136

43a. Biparental Inheritance and the Production of Diversity by Conjugation, in Chlamydomonag • . . . . . . . . 156

44. A Family of Paramecium Caudatum, Descended from an Exconjogant, and Showing Hereditary Abnormanties . . 160

45. The Separation of the Two Groups of Paired Chromosomes into Different Germ Cells, in the Insect Nezara Hilaris. . 178

46. The Chromosomes, and Their Separation into Two Nuclei with Reduced Numbers in the Protozonn Monocystis Rostrata 
FIGURE

PAGE

47. Reduction of the Number of Chromosomes at Conjugation in Didinium Nasutum • • • • • • • • • • • • 178

48. Conjugation and Reduction in the Number of Chromosomes in the Infusorian Anoplophrya Branchiarum . • . . 180

49. The Chromosomes and the Divisions Preparatory to Mating in Paramecium Caudatum . . . . . . . . . . . . 182

50. Reduction of the Number of Chromosomes Before Mating in the Rhizopod Pelomyxa . . . . . . . . . . . . 183

51. Diagram to Illustrate How Mendelian Inheritance Would

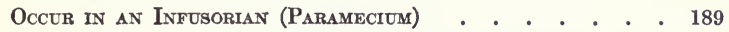

52. Conjugants and Non-conjugants from a Culture Composed of a Mixture of Two Races of Different Size, of Paramecium Aurelia. . . . . . . . . . . . . . . . . 191

53. Pairs from a Single Race of Paramecium Aurelia, Illustrating Assortative Mating . . . . . . . . . . . . 192 



\section{LIFE AND DEATH}

HEREDITY AND EVOLUTION IN UNICELLULAR ORGANISMS 



\section{LIFE AND DEATH \\ HEREDITY AND EVOLUTION IN UNICELLULAR ORGANISMS}

General Survey of the Life History in the Protozoa, with the Questions it Raises. Life, Death, Reproduction, Mating, Rejuvenescence, "Potential Immortality."

I ONG ago, when Latin was the common language of science, there was current a saying that nature is greatest in the things that are smallest: Natura maxima in minimis. Radl, in his History of Biological Theories, cites this as a maxim which led to mere superficiality; to the degeneration of biology toward an amusement, with the microscope as its instrument; to neglect of the really great problems of life. The naïve literature of the old fashioned microscopical societies, with their talk of the "golden tube," and of the "Oh, my! objects," passed from one member to another for the consecutive delectation of their eyes, lends some plausibility to this indictment; even yet the drama of life seen in a drop of water has its purely æsthetic fascination. But such fascination is not inevitably inconsistent with a serious value for studies of these creatures; there still exist students who, in spite of Radl's sarcasm, believe that the simpler creatures have something to teach us on even the deepest problems of life. I am going to try to 
present what we have thus far learned from them on the great questions of life and death; of heredity and evolution. I hope that this may serve as an introduction and foundation to a general understanding of these things; and that we may even find that the simplest organisms have a distinctive and important contribution to make toward such understanding. Study of what actually occurs even to its smallest details does indeed hamper uncomfortably the sweep of untrammeled theory, but if our aim is to attain truths that are verifiable rather than theories that are magnificently free, we shall welcome this result.

Let us set forth clearly at the beginning, that in these lectures our interest will not be primarily in Protozoology, but in Genetics: in the problems of life, its continuance and reproduction; and that we deal with the lower organisms only for the light they throw on these matters. With many of the technical problems which most interest Protozoologists we shall therefore have no dealings; on the other hand, the facts which we use will be such as do form constituent parts of the structure of Protozoology.

The traditional ground for hoping that the Protozoa may aid greatly in understanding the foundations of life and reproduction is this: As we pass from the complex organisms to the simpler ones, we must find that life retains its essential nature-for otherwise it would not be life-while stripping off all merely adventitious details. The highest organisms are of interest to us because they show the heights to which life may rise; the lowest because they show the fundamentals of life relatively unconfused. These lowest organisms are commonly said to consist of a single cell; whereas higher ones consist of an almost infinite number of cells of diverse kinds.

This point of view has been challenged in recent times, 
as well as in earlier years. It is admitted that some of these lower creatures appear to be extraordinarily simple as compared with trees, birds and men: such are evidently the bacteria among plants; such are amœba and its relatives among animals (see Figure 1). But in some of the Proto-
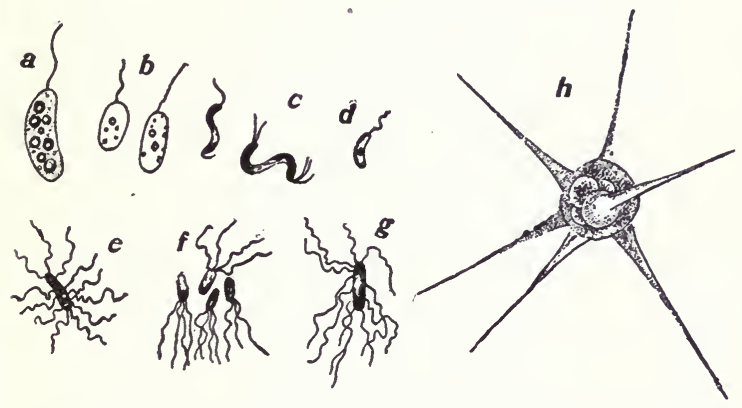

Figure 1. Simpler forms of Protista. $a$ to $g$, Diverse kinds of bacteria, after Zopf, Engelmann and Fischer. $h$, Amoeba radiosa, after Leidy.

zoa we find an astonishing complexity of structure (Figure 2, Figure 3) and of function, so that some of the students of the group, like Dobell (1911), maintain that there is no ground for calling these organisms "simple" or "lower" or even "unicellular": that they are merely small organisms, diverse in their plan of structure from the larger animals and plants. But it appears evident that amœbæ and bacteria are structurally simpler than vertebrates, in that they consists of fewer kinds of differentiated parts; and this seems to me true even for the more complex Protozoa, when compared with the Metazoa. They may therefore be properly called simpler or lower organisms, meaning thereby that they have fewer differentiated parts than the higher 

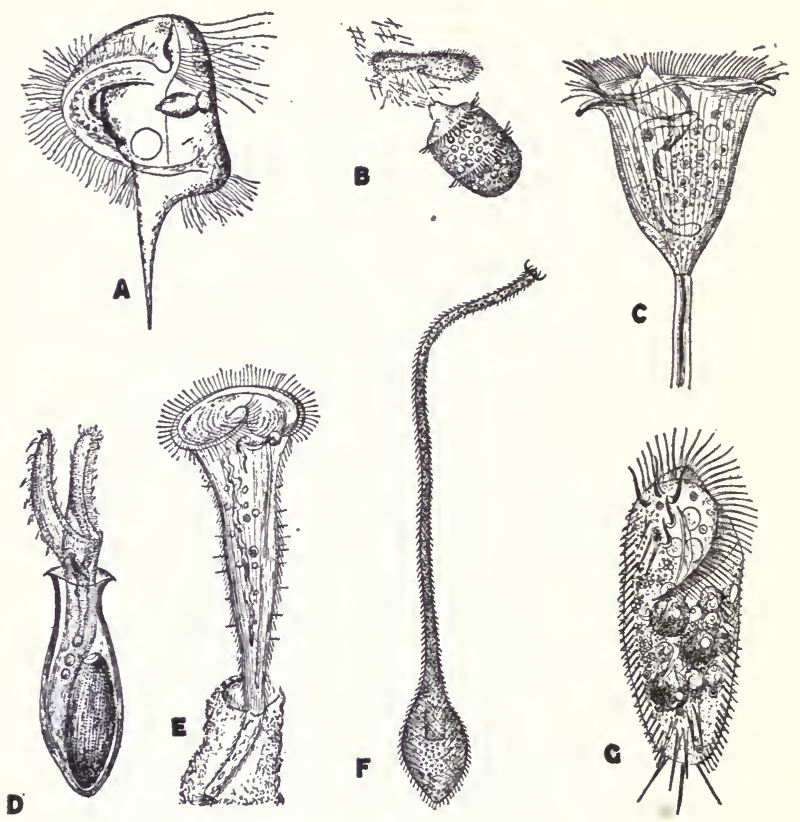

Figure 2. Some of the more complex Protozoa. A, Caenomorpha medusula Perty, after Bütschli. B, Didinium capturing prey, after Balbiani. C, Vorticella nebulifera O. F. M., after Bütschli. D, Folliculina, after Möbius. E, Stentor roeselii Ehr., after Stein. F, Lacrymaria olor, after Verworn. G, Stylonychia mytilus Ehr., after Engelmann.

animals. The interest of their study lies partly in discovering what life can do with few differentiated structures. Moreover, it seems clear that in their general plan of structure they are comparable to single cells of higher animals, though, like such single cells elsewhere, they may be very 


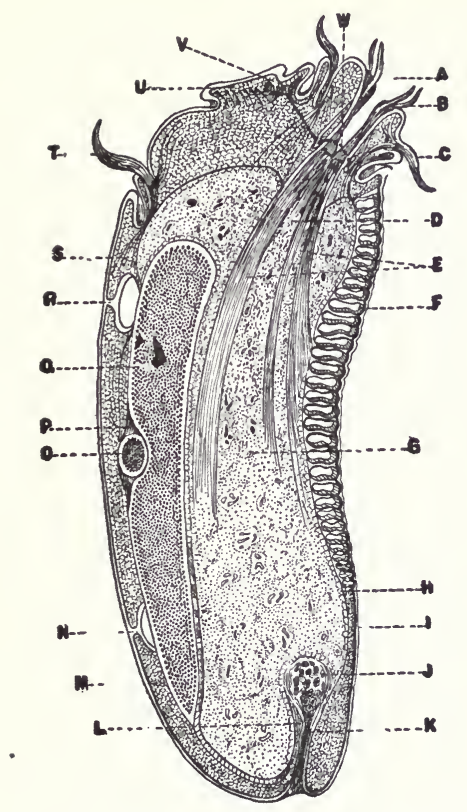

Figure 3. Structure of one of the most complex of the Protozoa, Diplodinium ecaudatum Fiorentini, after Sharp (1914). A, mouth; B, oral cilia; C, adoral membranelles; D, oesophagus; E, oesophageal retractor strands; $F$, ventral skeletal lamellae; $G$, entoplasm; $H$, ectoplasmic boundary layer; I, cuticle; J, caecum; K, rectum; L, rectal fibers; $\mathrm{M}$, ectoplasm; $\mathrm{N}$, posterior contractile vacuole; $\mathrm{O}$, micronucleus; $\mathrm{P}$, suspensory fibers; $\mathbf{Q}$, macronucleus; $\mathbf{R}$, anterior contractile vacuole; $\mathrm{S}$, posterior ciliary roots; T, dorsal membranellae; $\mathrm{U}$, operculum; V, motor mass; $W$, circumoesophageal ring.

complex; they may properly therefore be called unicellular organisms.

But whatever the facts as to their simplicity or complex- 
ity, they do show us life and the passage of generations following a very different course from that which we see in ourselves; a course which opens to us many new vistas. And they do present us a world of life in miniature in which structures are condensed into small space, in which activities are condensed into brief time. In a watch-glass on our table we may in a week see generations come and go; may observe the rise of whole faunas and floras; their decline and replacement by others; we may follow in successive generations the struggle for existence; may see natural selection at work and discover its results. In a few days we may see the birth, babyhood, youth and age of individuals, and their replacement by descendants; we may study the inheritance of parental traits by the new generation, or the appearance of new traits; we may observe how the population changes with the passage of ages,-and all while we wait for one of the changes of the moon.

This seems to present a wonderful opportunity for solving in a short time some of the problems at which men have been at work for ages, so that students have taken up this work with enthusiasm. But unhappily, when we cease to regard it as a mere fascinating spectacle, and desire to establish, in the rigid and detailed manner required by science, just what is happening in these teeming populations, and just what are the laws that the events follow, we find the difficulties very great, and our success perhaps less rapid than we might hope. For to do this we have to come to know these creatures individually; we have to work with them as we would with guinea pigs or with calves. The distance of size between them and ourselves is almost as difficult to overpass as are the spatial distances between us and the stars, so that studying them individually is a little like trying to get acquainted with the inhabitants of Mars. To 
become personally intimate with particular amobæ or infusoria; to control their goings out and comings in; their diet and personal habits; to interfere with their social and domestic relations; to feed them and mate them; to make them do and live as we want them to live,we.have to do if we are to really understand their lives, their behavior, their growth, their matings, their heredity, their evolution.

Some years ago I devoted myself to obtaining an intimate personal acquaintance with the life and behavior of individuals among these creatures; to study of their individual biography and perhaps psychology. I had the honor to present this to the public in a book on the Behavior of Lower Organisms. Building on the experience thus gained, I have since devoted myself to what happens in the passage of generations in these creatures; to a study of the biology of races rather than of individuals; to life, death, mating, generation, heredity, variation and evolution in the Protista. I am going to attempt to present a picture of these matters so far as our present knowledge makes possible. We shall find that there are still many questions which are not yet answered, but unsolved problems after all have their fascination; and much has been learned; this I shall try to present and compare with what is known for higher organisms.

When we follow the lives of particular individuals in the miniature jungle which a protozoan culture presents, we come upon a fact that is most astonishing to one who knows only the higher organisms. The creatures seem never to die, save by accident. If we follow a single individual, we find that after a time he divides into two (Figure 4). Which is the parent, which the offspring? Each of these again divides into two, and this continues for generation after generation. Nowhere does a corpse appear; nothing 
dead is found in the entire history of a race, save by accident. The present living generation transforms directly into the next one. Figure 5 illustrates the course of life and the passage of generations in these creatures as compared with that of higher organisms.

All this is so different from what we ourselves experience

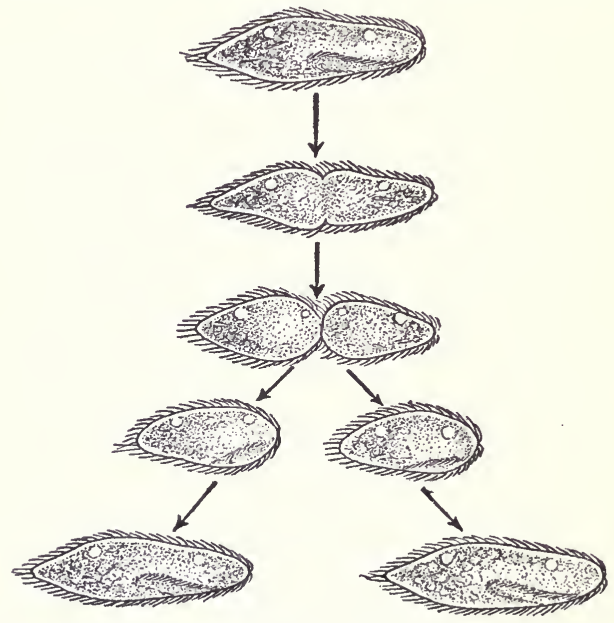

Figure 4. Division of an infusorian, Paramecium. Successive stages.

and from what we see around us in the animals that we know, that it has always aroused the greatest interest and led to many questions. What! are these creatures really immortal? Is there no decline, no old age, no natural death in their history? Does their life realize the dream of perpetual youth? Such questions, as you know, were put long ago by the early students of these creatures, and for 
almost a century the answers have been sought, through speculation and reasoning, through observation and experiment. After much conflict of opinion and many changes

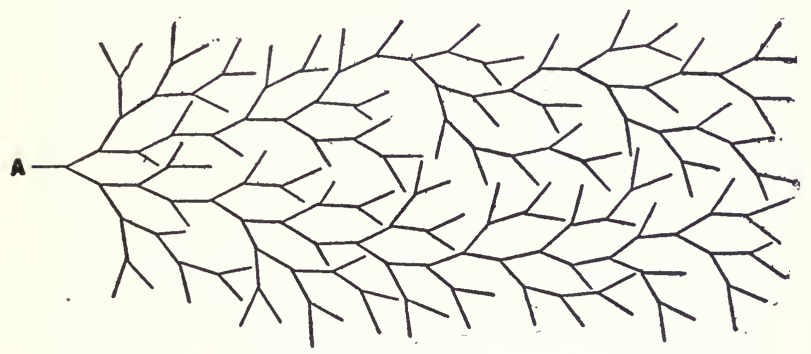

B

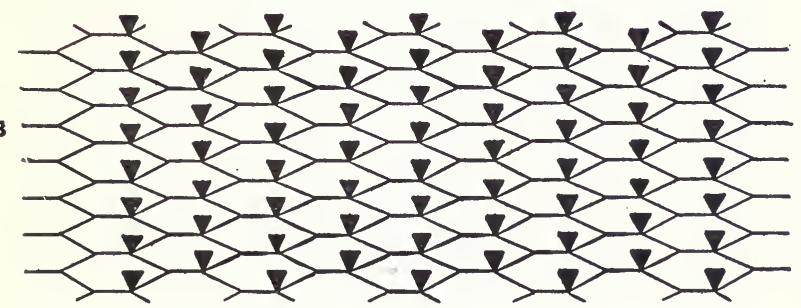

Figure 5. Diagram of the descent of generations in Protozoa or other organisms reproducing from a single parent; as compared with that in organisms reproducing from two parents. The lines represent the lives of individuals or germ cells, beginning at the left and passing to the right. $A$, uniparental reproduction by fission. The line of ancestry traced back from any individual at the right is always single; and there is no corpse found at any point, the present body transforming directly into the bodies of the next generation. $B$, biparental reproduction by union of germ cells, as in the higher organisms. The triangular structures are the bodies, the lines the germ cells. The line of ancestry from any individual traced back from the right forks at each generation, becoming in a few generations multiplex. The bodies of any generation are not continuous with the bodies of the previous generation-the latter dying, while the new bodies are produced by germ cells from two diverse lines. 
in the prevalent views, I believe that the last few years have brought us the facts which answer these questions. It is of these that I wish first to speak; they form a foundation for all our knowledge of these matters in Protozoa.

Although it appears that these creatures do not naturally die, it is equally apparent that they do reproduce. Every day or two, or even more frequently, each individual divides, and we have two where there was but one; in a few days their number has multiplied enormously. And if we watch them long and closely enough, we make another discovery of great interest: after the passage of many generations these creatures mate, as do higher animals and plants. Two individuals unite and exchange parts of their bodies (figure 6 ), then separate and both continue to live and to reproduce as before.

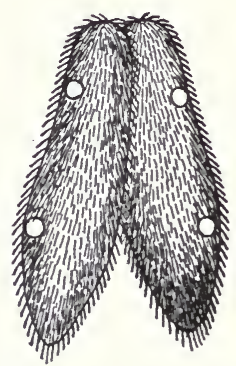

Figure 6. Conjugation of Paramecium.

Now if these creatures do not die, why should they mate and why should they reproduce? In ourselves and in the animals we know intimately, mating and reproduction are the prelude to death; reproduction seems a method of replacing the individuals that are to die. What is the use of these processes if those now alive are to continue to 
exist? Suppose that there were no death in man; there would be no need for reproduction; and if reproduction occurred it must soon result in overcrowding and in violent killing off on even a greater scale than occurred in the great war. And in these simple creatures the same overcrowding must result, for reproduction every twenty-four hours, without death, would in a few months pack all the waters of the earth with a solid mass of these creatures.

Reflecting thus on these things, many biologists came to the conclusion that these creatures must get old and die after all; that otherwise they would not mate and reproduce. We see indeed that not every individual gets old, for they continue to live and reproduce for generation after generation. So it was thought that it must be the passage of many generations that brings on age. The creatures, it was held, begin young and strong; they divide again and again, gradually getting old and weak; it is only the individuals of these later generations that show the weakness of age.

Now, since in higher animals mating and reproduction bring it about that an old and worn individual is replaced by one young and unworn, so, it was reasoned, must mating in these lower creatures cause an old individual to be replaced by a new one. But.when we observe the process, we do not see a new individual replace an old one after mating; apparently the old one continues to live and multiply as before. To avoid this difficulty it was concluded that in these creatures mating must rejuvenate the old individual; must in some way get rid of the age and wear, leaving the same individual, but physically young.

This was the famous theory of rejuvenescence through conjugation. It held that the young animal may live and reproduce for many generations without mating, till thou- 
sands of specimens have been produced from a single one. But as this goes on, they gradually lose their vitality, get old and decrepit, and must all in the course of time die, unless mating intervenes to save them. On the question of just what form this aging and decrepitude takes and just what are its symptoms, there was much difference of opinion. Bütschli and Balbiani believed that the power of reproduction gradually became less and less, so that division into two became less frequent; this idea was accepted by many, and has been maintained in recent years by Calkins; it is perhaps the prevalent form of the theory. On this view the occurrence of conjugation restored the reproductive power, so that fission now continued as rapidly as it did before the animals grew old. On the other hand, though the fact is not generally realized, some of the chicf upholders of the theory of rejuvenescence emphatically rejected this idea that aging showed itself in a decline of the rate of reproduction; this is notably the case with Maupas, who asserted positively that no such decline occurred before conjugation, and that after conjugation reproduction was no more energetic than before; and Richard Hertwig discovered experimentally that Maupas' statement is correct. They believed however that in other ways the animals become decrepit and that they must die if conjugation did not occur.

Now, just what is it that happens in mating which might be conceived to bring about rejuvenescence? There are two main things that occur in conjugation; to get these clearly in mind we must look for a moment at the structure of an infusorian, and at the process of conjugation.

An infusorian resembles in the plan of its structure a single cell of a higher organism, but has its nucleus in two parts (Figure \%, a). One of these parts is large and seems to be the active portion; the other is very minute and appears 
to be a sort of reserve of nuclear material. The large part, or macronucleus, seems to take a part in the physiological processes of the daily life of the cell. The reserve nucleus, or micronucleus, seems to lie inactive, save at the time when the creature divides; then both the large, active nucleus and the small reserve nucleus divide, so that both the new indi-
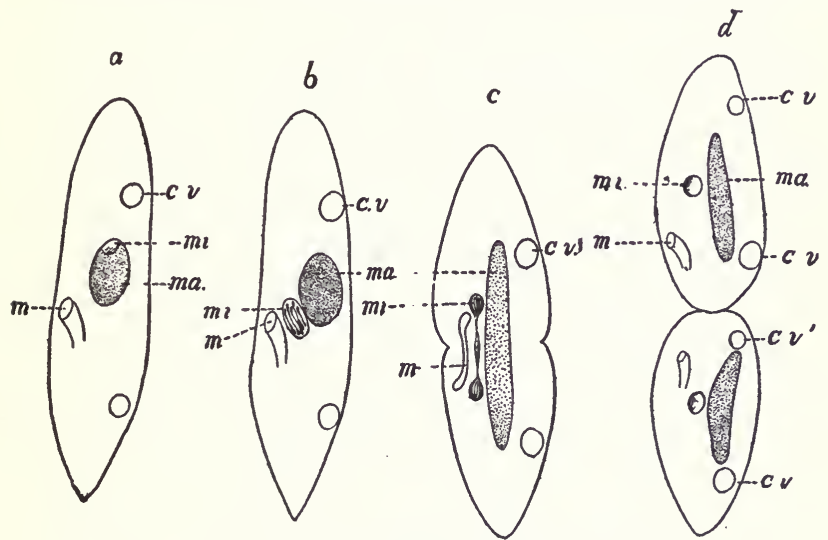

Figure 7. Diagram of the process of division in Paramecium; successive stages. c. v., Contractile vacuoles; m., Mouth; ma., Macronucleus; mi., Micronucleus.

viduals produced contain half of each (Figure 7, c, d). Sometimes there are several of the small reserve nuclei.

Now at the time of conjugation (Figure 8), in each of the mated individuals the old active nucleus breaks up and gradually disappears, being apparently absorbed like so much food, by the rest of the body. The reserve nucleus (mi., Figure 8), on the other hand, divides several times; in Paramecium caudatum, for example, each divides twice 
(A to C), so that four are produced. Three of these disappear, as did the old active nucleus, and the fourth divides into two halves (D). One of these halves passes over into
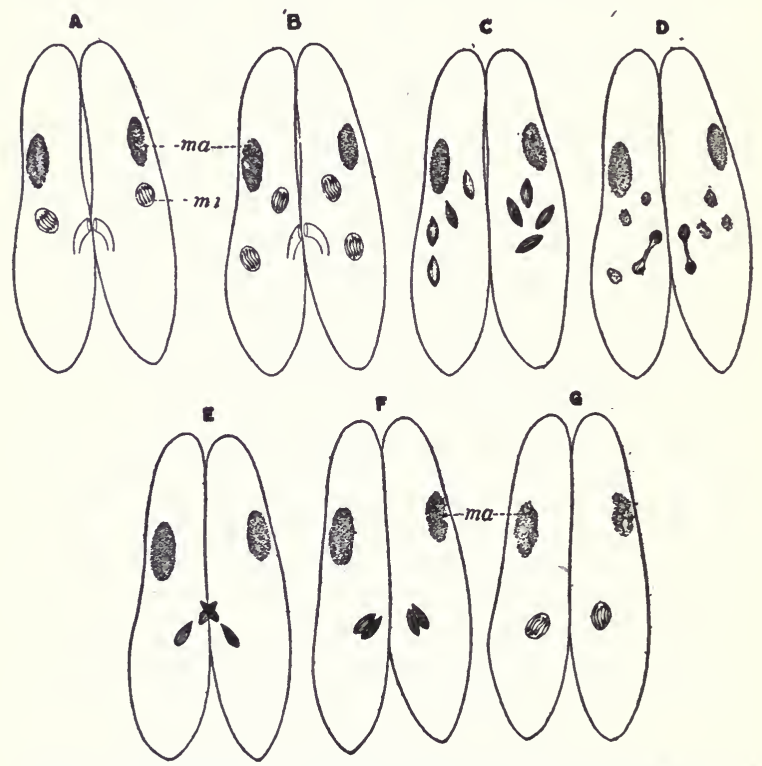

Figure 8. Diagram of the internal processes in the conjugation of Paramecium caudatum; successive stages. ma., Macronucleus; mi., Micronucleus. The striated and spindle-shaped bodies are the micronucleus in the process of division. In $\mathrm{D}$ the three smaller dotted clouds are three degenerating micronuclei.

the other individual, with which mating is occurring (Figure $8, \mathrm{E})$, where it unites with the corresponding remaining half of the reserve nucleus of that individual $(F, G)$. That is, the two conjugating animals exchange halves of their reserve 
nuclei, so that each has after mating a new reserve nucleus, composed half of reserve nuclear material from its own body, half of reserve nuclear material from the body of its mate (Figure 8, G.). Later this new reserve nucleus divides into parts, some of which become new, large, active macronuclei, while the others remain as minute micronuclei (see Figure 30, Lecture 5).

So there are two main things in conjugation: (1) The old active nucleus is replaced by parts of the reserve nuclei; (2) the two mating individuals exchange parts of their nuclear material.

Now, evidently the replacement of the old active nucleus by part of the reserve nucleus is just the sort of thing that one would expect if there is to be rejuvenescence; indeed, it is rejuvenescence of the macronucleus. It looks very much as if the old active nucleus might have gotten worn out or used up in its activity, so that it has to be replaced by reserve material which has not been used.

But what has all this to do with mating? If the point is merely the replacement of worn nuclear material by fresh material from the reserve store that each animal carries, why need there be this complicated process of exchange of nuclei? Rejuvenescence should occur just as well without this exchange, without conjugation, as with it.

The occurrence of these two distinct things at conjugation,-replacement from a reserve, and exchange,-has always kept the theory of rejuvenescence ambiguous. Is it the replacement from reserve material, or the exchange, that makes the organism young again? Authors have as a rule either not ventured to answer this question, or have not clearly analyzed the process into its two elements,-speaking merely of conjugation as a whole. Arguments based on the replacement process have been used or accepted as argu- 
ments referring to the process of exchange, and vice versa. What was needed was some method of separating the two processes, to see what effect each has by itself.

In the meantime, taking conjugation as it occurs, without this analysis, men attempted to find out: first, whether the organisms do grow old and die if conjugation does not occur; second, whether conjugation does save them, does make them young, does cause them to reproduce more energetically.

The first question, as to whether without conjugation the creatures degenerate and die, has cost an infinite amount of labor to generations of investigators. It appeared that as a matter of fact, if the animals were kept without conjugation they do die out in the course of time; such was the result of the long continued labors of Maupas (1888, 1889). After some hundreds of generations without conjugation the animals weakened, became abnormal, sickened and died. It is worth while to note Maupas' exact results on this point. We may give them in the words of his own summary :-

"I have kept six cultures until their final extinction by senile exhaustion. The first (Stylonychia pustulata) became extinct after 215 fissions; the second (Stylonychia pustulata) after 316 ; the third (Stylonychia mytilus) after 319 ; the fourth (Onychodromus grandis) after 320 to 330 ; the fifth (Oxytricha) after 320 to 330 ; the sixth finally (Leucophrys patula) after 660." (Maupas 1888, p. 260). ${ }^{1}$

But as other investigators took up the same sort of work, a curious fact was found. As men began to be able to

${ }^{1}$ It is worth noting that different infusoria showed great diversities as to length of life; Leucophrys patula lived in Maupas' experiments for twice as many generations as any of the other species. It should also be observed that Maupas did not carry out such experiments on Paramecium, the organism that has been most used for such work since his time. 
take better care of the animals; to give them proper food, and to vary their food, the organisms were found to live longer and longer without conjugation, and to give less indication of old age. Calkins (1904) kept the infusorian Paramecium caudatum for 742 generations without conjugation, but they finally weakened and died. Enriques (1903) kept Glaucoma scintillans for 683 generations without conjugation and with no sign of harmful effects; and he has recently (1916) kept Glaucoma pyriformis for ${ }^{2} \% 01$ generations with no sign of degeneration. And finally Woodruff (1917) has kept Paramecium aurelia for more than 6000 generations without conjugation; the stock at last accounts was still flourishing, with no sign of aging, of degeneration.

Now the fact that one can by proper methods of culture keep these creatures healthy for more than 6000 generations without conjugation, shows that the degeneration which came, under other methods of culture, in a few hundred generations, was not evidence that conjugation is required, but only that the culture methods were not good. It has been found that some species of infusoria cannot stand, save for a short time, the method of culture necessary if the separate generations are to be accurately counted; others can exist under these conditions for a greater number of generations; other indefinitely; and that without conjugation.

Thus the result of the work so far done has been to confirm the view that the infusoria may live indefinitely without mating. I believe that we may look upon this as one of the secure results of science. There are many of the unicellular creatures, particularly the bacteria, in which nothing like mating is known. It is sometimes held that such processes must yet be found in these creatures. But the fact that infusoria, which do mate, may nevertheless live 
indefinitely without mating, makes it probable that the appearances are correct, and that such organisms as bacteria actually never mate.

Does this confirm the theory of the "potential immortality" of these creatures? It apparently does, if we are to take that expression in its broadest meaning. But the real question underlying that phrase is this: Does the exercise of the functions of life itself necessarily result in deterioration, in senility, in final death? This was the question that Maupas believed his experiments to answer affirmatively. He says of his experiments, "They demonstrate that indeed in the ciliate infusoria, as in so many, if not all, other living things, the organism deteriorates, uses itself up, simply by the prolonged exercise of its functions" (1888, p. 261).

The experiments we have described above show that conjugation is not required to remedy the wearing away of the organism through the exercise of its vital functions. But is this the complete story? Is there after all no significance in the fact that these creatures keep a reserve nucleus, along with the active one? Does the active nucleus never require replacement from the reserve?

This question too has recently been answered, mainly by the work of Woodruff and Erdmann (1914). And in answering the question they have succeeded in observing that separation of the two processes in conjugation, of which we said above there was so much need. These two processes are: first, the replacement of the active nucleus from the reserve; second, the exchange of parts of the nuclei in mating. Woodruff and Erdmann observed the replacement process occurring without mating and exchange. And in doing this they have uncovered what is evidently one of the most important phenomena that occur in the life of these Protozoa; something that must form a background for all 
study of life and reproduction in these creatures. We must therefore examine it with care.

In their race of Paramecium that lived indefinitely without conjugation, Woodruff and Erdmann found that the active macronucleus is replaced at rather short intervals

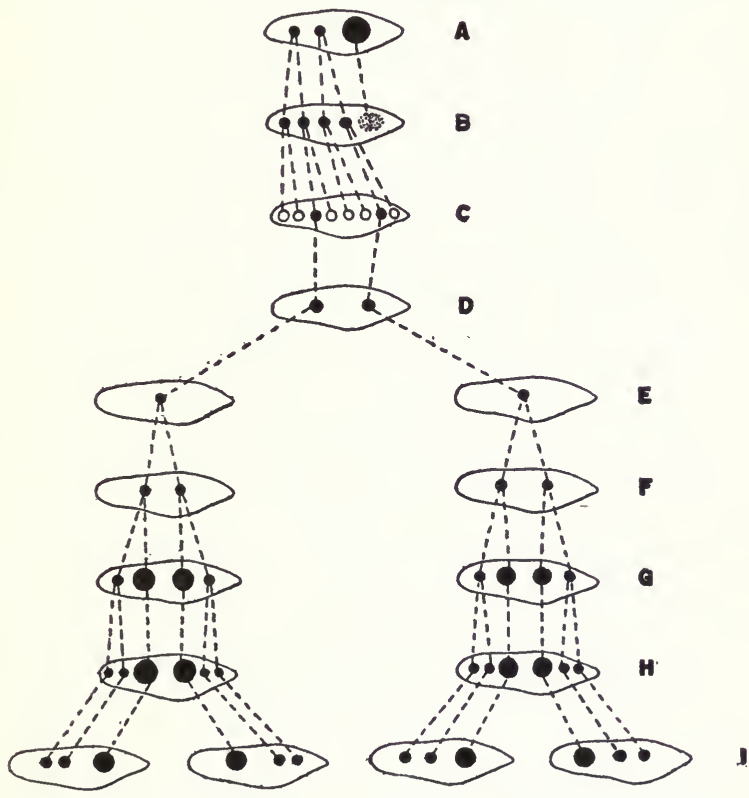

Figure 9. Diagram showing what occurs in endomixis, or the replacement of the old active macronucleus by a part of the reserve nucleus (micronucleus) ${ }^{-}$in Paramecium aurelia. Constructed from the figures and description of Woodruff and Erdmann.

The earliest stage (condition before the process begins) is shown at the top; successive later stages from above downward. The larger black bodies represent the macronuclei; the smaller ones the micronuclei. The clear circles are the micronuclei that degenerate. 
by parts of the reserve nucleus. Every forty to fifty generations the macronucleus breaks up and is absorbed and disappears, just as happens when conjugation is to occur. Then each reserve nucleus divides (in Paramecium aurelia) so as to produce eight (see Figure 9). Six (or seven) of these disappear by absorption, like the active nucleus. The remaining one later produces by division the new active nucleus (macronucleus), and the reserve nucleus or nuclei. That is, the active nucleus is replaced every forty or fifty generations by material from the reserve store. This whole process Woodruff and Erdmann call endomixis.

This process has been found to occur, not only in the single race in which Woodruff and Erdmann first found it, but in many other races, and in another species of Paramecium. And in another infusorian, Stylonychia, the Russian investigator, Fermor (1913), states that a similar process occurs at the time of encystment. After multiplying for five or six weeks the animals lose all their appendages and other organs, gather into a sphere and form a sort of thin shell about them. Then the two active nuclei disappear and are replaced from the reserve nuclei, which have united to form one. The very brief account of this process by Fermor was published earlier than the work of Woodruff and Erdmann. It has been stated by Calkins that a similar process occurs in the encystment of Didinium (1915 a), and in Uroleptus (1919). It seems probable that it will be found generally in the infusoria.

The discovery of this process of replacement of the active nucleus by the reserve nucleus evidently puts a new face on the matter. To the question whether the living substance uses itself up in functioning, so as to require replacement, it seems to answer "yes!" even more definitely than any discovery that conjugation was necessary would have done. 
The question becomes of extreme interest whether such a process as this is of general occurrence; whether it is necessary in order that aging and death shall not occur. Many investigations are therefore at this time directed upon endomixis. Certain questions must occur to everyone; some of these have already been answered.

First, is this replacement perhaps merely something brought about by unfavorable conditions of the environment, and not necessary if all conditions continue good? Jollos (1916), Young (1917), and Woodruff (1917) find that under unfavorable conditions the replacement of the active nucleus from the reserve is brought about more quickly than would otherwise occur. But under the most favorable conditions the process does not cease, and under uniform conditions it takes place at uniform intervals. Woodruff found that whenever the process of replacement ceases entirely, the race dies out. This evidence therefore indicates that the process is a necessity for continued life.

Enriques (1916) attempted to test the matter by studying an organism in which there were no periods of slow fission, such as are found while the replacement is occurring. He found such an infusorian in Glaucoma pyriformis, which under the conditions he used may produce 10 to 13 generations a day, and continue this without interruption. $\mathrm{He}$ kept this organism for 2701 generations, or for more than 8 months, during which there were not less than 9 generations every single day. The culture was still in progress at last accounts; there was no sign of degeneration, and no periods of slow fission. Enriques therefore holds that endomixis has not occurred. He concludes that endomixis is not necessary to continued life, just as conjugation is not.

It is evident however that this conclusion is insecure; it is not impossible that endomixis occurred without interrupt- 
ing the regular series of fissions. The matter requires actual cytological study before we can know whether or not anything of the sort occurs in Glaucoma.

In Paramecium, Woodruff and Erdmann (1914) found that while the replacement is going on, and for a little before and after, the rate of reproduction is slower than at other times. Does this mean a waning of vitality, which is corrected by the replacement? Jollos (1916) found that under favorable and uniform conditions, the slowing of the fission rate is limited sharply to the period in which the processes of replacement are occurring. This slowing of cell division would therefore appear to be merely a natural result of the fact that while the complicated changes of endomixis are occurring, the organism does not so readily undergo at the same time the involved processes of fission. It is therefore not clear that there is any indication of loss of vigor, of senile changes, setting in before each replacement occurs. The process seems to be carried on, like many others in nature, with such a "margin of safety" that there is no indication of exhaustion before it occurs; it takes place before there is a pressing need for it.

Thus it appears that in these organisms nature has employed the method of keeping on hand a reserve stock of a material essential to life; by replacing at intervals the worn out material with this reserve, the animals are kept in a state of perpetual vigor; not, as individuals, growing old or dying a natural death. Nevertheless, a wearing out process, such as might be called getting old, does occur in the structures employed in the active functions of life, and these must be replaced after a time of service. So far as the conditions in these organisms are typical, deterioration and death do appear to be a consequence of full and active life; life carries within itself the seeds of death. It is not mating with an- 
other individual that avoids this end; but replacement of the worn material by a reserve.

It results that the continuity of life in the infusoria is in principle much like that in ourselves, though with differences in details. As individuals, the infusoria do not die, save by accident. Those that we now see under our microscopes have been living ever since the beginnings of life; they come from division of previously existing individuals. But in just the same sense, it is true for ourselves that everyone that is alive now has been alive since the beginning of life. This truth applies at least to our bodies that are alive now ; every cell of all our bodies is a piece of one or more cells that existed earlier, and thus our entire body can be traced in an unbroken chain as far back into time as life goes (see the diagram, Figure 5). The difference is that in man and other higher organisms there have been left all along the way great masses of cells that did not continue to live. These masses that wore out and died are what we call the bodies of the persons of earlier generations; but our own bodies are not descended by cell division from these; they are the continuation of cells that have kept on living and multiplying from the earliest times, just as have the existing infusoria. From our own personal point of view it seems unfortunate that the mass of cells which is next to wear out and be left behind in the chain of life is that with which our own selves seem to be bound up; but certain samples of ourselves may continue to live indefinitely, like the infusorian. The great mass of cells subject to death in the higher animals dwindles in the infusorian to the macronucleus; this alone represents a corpse. But the dissolution of this corpse occurs within the living body. It resembles much such a process as the wasting away and destruction of minute parts of our own bodies, which we know is taking place at all 
times and which does not interrupt the life of the individual.

What now are we to think of conjugation in the light of these new facts? In conjugation there occurs the same replacement of the old active nucleus by a part of the reserve, which we have seen to take place also without conjugation; but with the additional fact of an exchange of pieces of the new nucleus between the two conjugating individuals. If this replacement means rejuvenescence when it occurs without conjugation, there is no reason to deny it that meaning in conjugation; conjugation too should result in rejuvenescence. But what is the significance of the additional feature - the exchange of nuclear parts between the two conjugating animals? This seems not necessary to rejuvenescence, since the replacement of the old by the reserve may occur without it. Why then does the exchange take place?

In many higher organisms we observe that the mating and the union of parts of two different cells which we call fertilization are necessary in order that reproduction shall occur; the egg does not develop unless fertilized. But in these Protozoa we observe that reproduction occurs for generation after generation without this mixture of two diverse cells; and we have just seen that rejuvenescence likewise occurs without such a mixture. In these lower creatures we find separated therefore two processes which in many higher animals are so closely bound together that we get the impression that they are inseparable; that development necessarily depends on fertilization. But even in many higher animals development may take place without fertilization; these, taken with the facts in the Protozoa, show that there is no generally necessary relation between the two things. It appears, as we shall see later, that the close association of reproduction with a union of two cells is only a special peculiarity of certain organisms: something that might be called a special adaptation. 
What ground then can we possibly give for the interchange of parts of the two individuals that conjugate? We shall take up this question in all its aspects later; here I wish to bring up but one of the possibilities. We observe that after the two individuals have conjugated and separated, they are no longer just what they were before. Each is now formed of parts of two individuals, - a body and half the nucleus from one; half the nucleus from the other. Will the two individuals therefore now be diverse in other respects from what they were before? Will their general characteristics be changed? Will they behave differently; will they develop differently; will they produce young of a different sort? In other words, are the other characteristics of the two individuals mixed as well as their nuclei?

This we know is what happens as a result of fertilization in higher organisms; the young produced inherit from both parents. Does it happen also in the Protozoa? If so, it will give us an understanding of the exchange of parts in mating.

This raises for us the problem of heredity in the Protozoa. Do the young produced by any given parent inherit also from the individual with which that parent has mated?

But this is heredity in its most complex forni. In the Protozoa, as we have seen, we have, for generation after generation, reproduction from a single parent by simple division. This presents the problem of heredity, and also those of variation and evolution, in the simplest possible form, and we shall do well to study the problems here before we take them up in cases where two parents are involved. We shall therefore examine this matter in our next lecture, and later take up the entire natural history of mating. 
Heredity and Variation in Protozoa, in Reproduction from a Single Parent. "The Inheritance of Acquired Characters." The Existence of Many Diverse Stocks in a Single Species. Constancy of the Stocks. "Pure Line Inheritance" and the Results of Selection. Difficulties for the Theory of Evolution.

UR first chapter led us to the question of heredity in the Protozoa. In the present chapter we take up the study of heredity and variation in the simplest kind of reproduction, where the offspring are produced by the division of a single individual into two. By way of introduction, let us bring this into relation with the problem of life and death, which we have already considered. We have seen that these organisms are so constituted that they live indefinitely, with no intervention of natural death of the individuals; yet we have seen also that they continue to reproduce. The inevitable result is that more individuals are produced in each species than nature can provide space and opportunity for, so that most of them are condemned to violent and unnatural death. Is there anything changed by this continual over-production, with destruction of the majority? Are those produced exactly like those that existed before? Or do the animals change as generations pass, so that some are better fitted for the conditions that they meet, and therefore continue to exist, while others are killed off? That is, can we see evolution occur as we watch these creatures through generation after generation? 
Turning now to the facts, we know that when one of these animals reproduces, it merely divides into two; the parent simply transforms into two offspring. Are the two just like the parent from which they came? When we

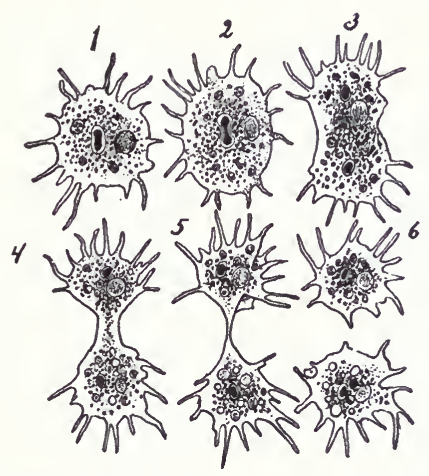

Figure 10. Reproduction in Amoeba; successive stages. After F, E. Schulze.

examine the facts in amœba (Figure 10), we can see no reason why they should not be; they merely are the parent, but now in two parts; the difference in size between the formerly existing parent and its two progeny is quickly remedied by a little growth. So at first view the problem of heredity seems in these creatures absolutely simple; the progeny are the parents, merely divided; so they must be like the parents.

But if we don't stop at amœba, but examine other Protozoa also, we find that the matter is not so entirely simple after all; indeed, we find that heredity presents the same problems that it does in higher animals. Take for example 
a close relative of amœba,-Difflugia (Figure 11), which is merely an amœba with a shell. In reproduction the two products do not receive half the parental shell; if they did, as you can readily see, they would indeed not be like the
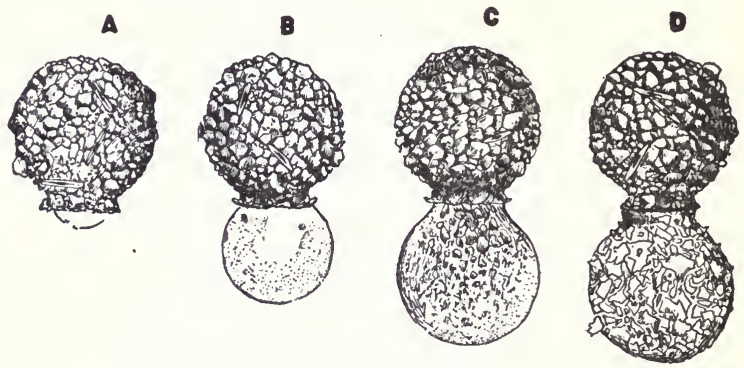

Figure 11. Reproduction in Difflugia. After Verworn (1888).

parent. What happens is this: The shell consists of sand grains, embedded in a hard chitinous substance. As the parent creeps about in its daily life, it takes up sand grains and stores them in the interior of its body. At reproduction the protoplasm of the parent swells and projects from the mouth of its shell (Figure 11, A). This projecting mass takes a form similar to that of the parent (B). The sand grains within the parent body pass out into the projecting mass, come to its surface, and spread over it (C, D). They are embedded in a fluid secretion which now turns hard, so that they form a shell like that of the parent. The two. shells are in contact at their mouths (D). Now the common mass of protoplasms divides into two, and the two individuals separate-one retaining the old shell; the other with the new one.

Now you see that it is by no means a simple matter of 
course that the new individual should have a shell just like that of the parent. Its shell is produced anew. The parent shell may have a peculiar form or structure; in some cases
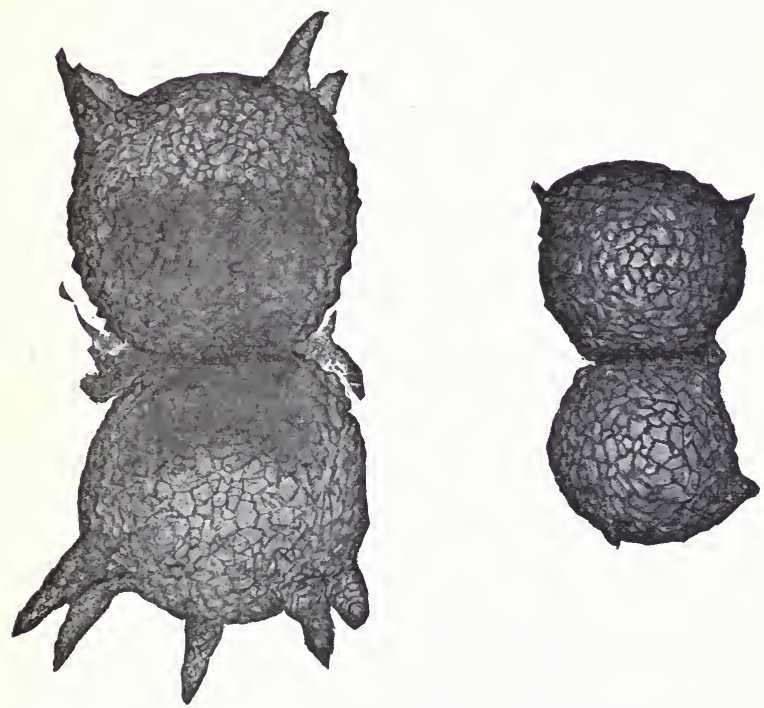

Figure 12. Two parents, with their offspring, just before separation, in Difflugia corona. The parents are above, the offspring (slightly lighter in shade) below. After Jennings, 1916.

for example, as you see in Figure 12, it bears spines of a certain length and form and in a certain number. There is no simple evident reason why the progeny should produce a shell of the same form, with spines of the same length and number. These things are not by any means merely handed on bodily from parent to offspring. On the contrary, just 
as in higher animals and ourselves, the progeny produce anew their peculiar characteristics. The problem of heredity is :-Why should they produce the same sort of characteristics that their parents produced? The problem is of just the same sort in the Protozoa that it is in ourselves. In such a case as Difflugia the answer which suggests itself takes something of this form: the offspring produce the same sort of shell that the parents did, because they are made of the same sort of material. This answer is indeed little but a form, but it is a form into which a more complete answer will have to fit.

Before inquiring further as to just how closely the offspring do produce the same characteristics as the parents, let us look at another example or two, showing in a still more marked way the nature of inheritance. In some of the more complex Protozoa, such as the hypotrichous infusoria, we find that the body bears a great number of organs of definite form and number, arranged in a precise manner. Observe in this Stylonychia (Figure 13), the numerous leglike or fin-like appendages, for creeping or swimming. When the animal reproduces, it divides cross-wise, and if it merely divided without any rearrangement of parts, you can see that the two progeny would be most unlike the parents. As a matter of fact, during reproduction all these organs disappear; they are apparently gradually absorbed into the body. Then on each half of the body there appears (even before the disappearance of the old organs) a new group of minute projections (Figure 13, B), all close together; not arranged at all as were the appendages of the parent, and not showing the differences of size and structure that were found in the parent. These small projections now proceed to change place, scatter themselves, and take up positions corresponding to those of the appendages of the 
parent (Figure 13, C); at the same time they transform in various ways, till they have produced anew a set of organs like those of the parent, of the same number, form, size and arrangement.
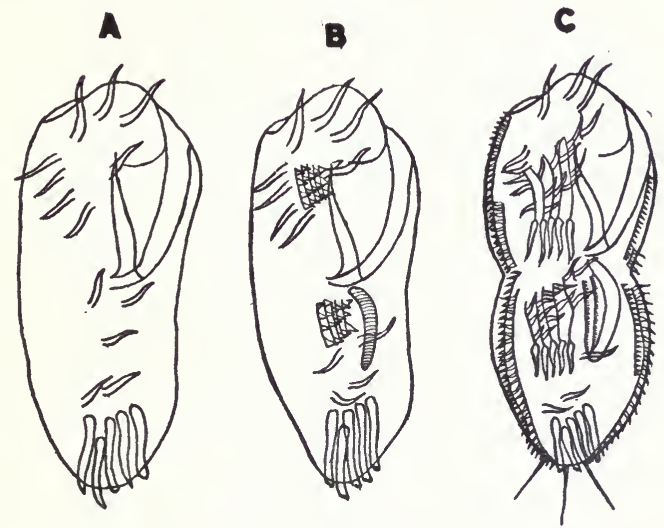

Figure 13. Reproduction in Stylonychia, after Wallengren (1901). A, Parent before reproduction. B, Appearance of the two groups of small projections that are to form the appendages of the two offspring. C, Division is occurring; the two groups of embryonic appendages are scattering out to take up their final positions. The old appendages have not yet disappeared.

All this illustrates the general nature of the problem of heredity in both lower and higher organisms; the features which the offspring are said to inherit from the parents they really produce anew for themselves, and the problem is as to why and to what extent they produce the same things that the parents did.

There are certain exceptions to this general rule that the progeny have to produce anew what they inherit; a few 
things are directly handed on from parent to offspring. These few things are of great importance, for it is these that in some way provide the foundation for the new production of the other structures. In the Protozoa these are as follows: (1) The halves of the nuclei; in cases where there exist separate active nuclei and reserve nuclei (macroand micro-nuclei), a half of each of these is passed on to each of the progeny (see Figure \%). (2) Secondly, a half of the general protoplasmic mass, or cytoplasm, goes to each of the progeny. As we have seen, the particular organs borne by this mass usually are not handed on bodily, but first disappear and are then produced anew by the offspring. Some few definite organs are in particular cases passed on bodily, but even in these cases at least half of them must be produced anew,-else of course they would occur only in half as great number in each of the two offspring. All the precision of the process of division and handing on is seen in the nucleus (see Figure 49, page 182), so that it seems probable that its different parts, each so accurately divided, have special and diverse functions to perform in the production of the new organs of the progeny. Just what part is played by each of these things that are directly handed on from parent to offspring, in producing the final characters of the progeny, is one of the chief questions of heredity.

When we have gotten to our present point in the examination of reproduction and heredity in these creatures, we shall not make the mistake of some of the earlier writers, as to the inheritance of acquired characters in the Protozoa. By acquired characters we mean characters that an individual did not inherit from its parents, but which were produced by special conditions during its life. Since the parents merely divide and become the offspring, it was set forth as a 
matter of course that in the Protozoa the offspring would receive the acquired characters as well as the other characters of the parent. But as we have seen, it does not receive even the other characters of the parent; but produces them anew. Is there any reason why the offspring should
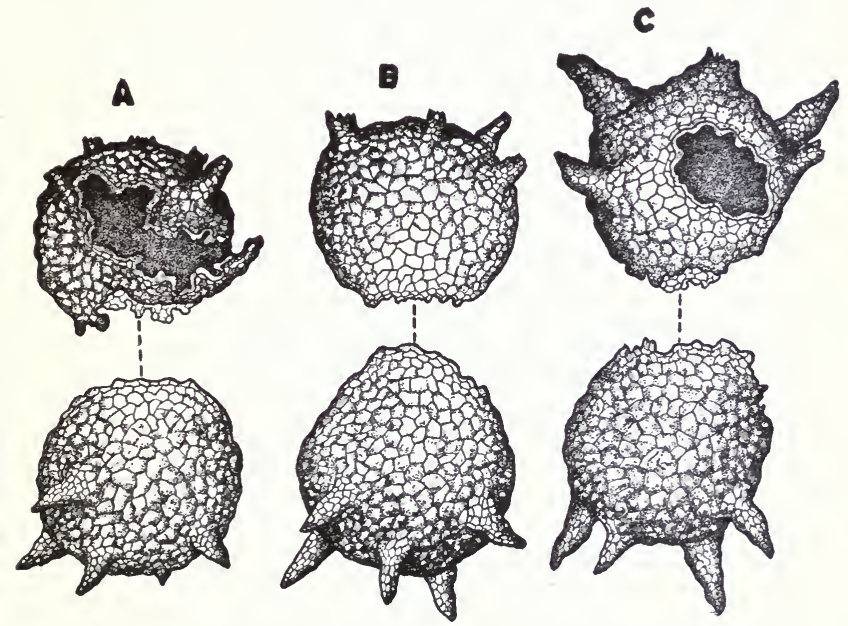

Figure 14. Inheritance from mutilated parents, in Difflugia corona. The three parents, A, B and C, have had their shells and spines broken. The offspring of each is seen below it, with normal, unmutilated shells and spines. The mutilations of the parents are not inherited by the offspring. (From observations by the author.)

produce anew characters that the parent has acquired merely accidentally, owing to special circumstances? Let us look at a few of these. There is no place so favorable for getting in a simple way a clear idea of the problems and difficulties involved in the "inheritance of acquired characters" as in the Protozoa. To many persons who have not examined the 
details it seems extraordinary that anyone should doubt that acquired characters are inherited.

Let us examine first the simplest possible case, of an acquired injury or mutilation. Suppose that in Difflugia one or more of the spines is broken off, or a hole is broken in the shell (Figure 14). Is there any reason why when this animal reproduces, the progeny should have a corresponding broken spine or broken shell? The off spring produces anew

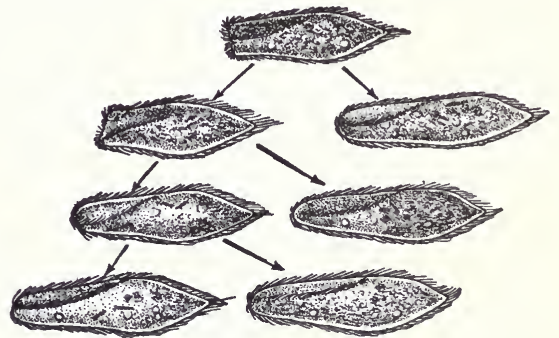

Figure 15. Reproduction in an infusorian (Paramecium) in which the anterior end has been cut off. At the first division the posterior offspring is quite normal, and by the third division even the anterior offspring has regained its normal anterior tip. Only the divisions of the mutilated individual are represented, as all others produce normal offspring. After Jennings, 1908.

its own shell and spines; what sort it shall produce under given outer conditions depends on the nature of its protoplasm and nucleus. These are not altered by the breaking of the shell or spine of the parent, and as a matter of fact we find that the offspring produce entire shells and spines, just as their parents did. The injuries acquired by the parent are not inherited. Again, look at an infusorian with its anterior tip cut off (Figure 15). Is there any reason why the off spring produced from the hinder half should have its anterior tip cut off? As a matter of fact, it has not. 


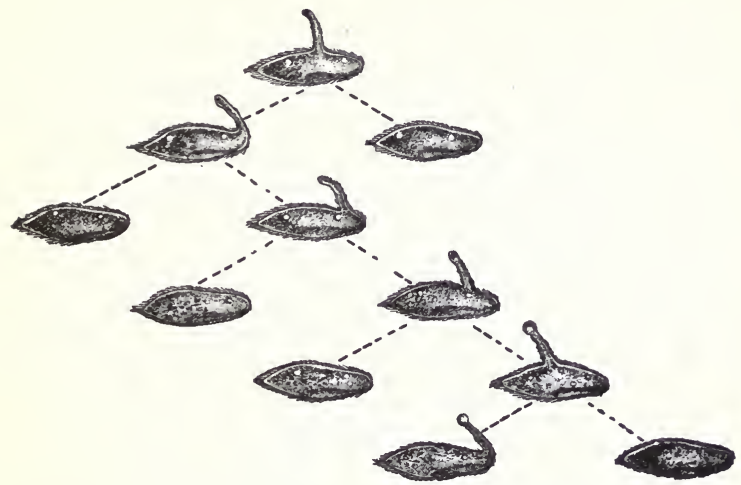

Figure 16. Reproduction for several generations of a Paramecium bearing a large projection on its aboral surface. At each division only one individual receives the projection; all other offspring and descendants are normal. After Jennings, 1908.

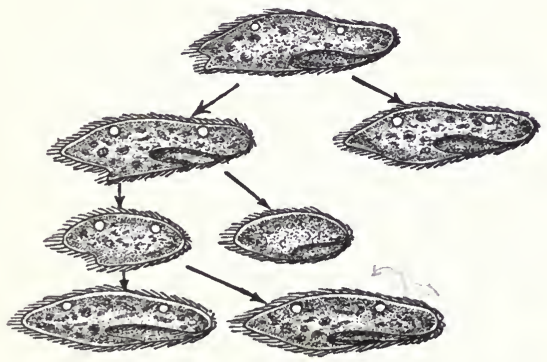

Figure 17. Reproduction in a Paramecium bearing a small abnormal projection near the posterior end. All offspring but one are normal from the beginning, and even the abnormal individual becomes normal after two divisions. After Jennings, 1908. 
Or observe an infusorian in which a new structure-a projection for example-has been produced through some accident (Figure 16). Will this thing be inherited at reproduction? It will naturally be carried on mechanically by a single one of the individuals at reproduction, but the rest do not produce it. After a thousand new individuals have been produced, one of them may still have the new structure, but no more than one. As a matter of fact, such a new structure is usually gotten rid of completely in the making over that accompanies reproduction (Figure 17). I have

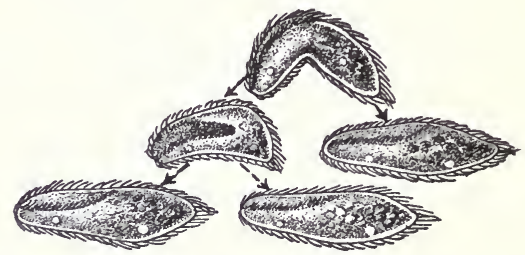

Figure 18. Reproduction in a deformed individual of Paramecium. After the second division all descendants are normally formed. After Jennings, 1908.

examined a large number of such cases in the infusorian Paramecium; the acquired peculiarities are not inherited (see Figures 15 to 18 ).

So there is no simple direct inheritance of acquired characters in the Protozoa, any more than there is in the higher organisms. The progeny have to produce the characters that they get, just as the parents did,-and they usually produce what the parent produced when it developed,- - not what the parent may accidentally happen to have when it divides. The progeny start where the parent did, as a rule.

Of course if we could get the fundamental constitution of 
the organism to change- say the chemical nature of its nucleus and cytoplasm-we might expect it to develop in a new way, and produce new structures; and as this would be repeated in later generations, we should have the new characters inherited. But this is a deep and difficult matter; we shall take it up later. What we wish to bring out now is the fact that the mere existence of an acquired character in the parent presents no reason for expecting the progeny to produce that character anew, and that as a matter of fact they do not; such acquired characters are not inherited, either in Protozoa or in more complex organisms.

We have seen thus that the progeny do not receive their organs ready made from the parent; that on the contrary they start from just the same condition the parents didan undifferentiated condition without organs, on the whole - and produce their characteristics anew. The ground for their producing the same characteristics as the parents lies precisely in this fact, that they start just as the parents did.

Having thus examined the groundwork for inheritance, we wish to look at the matter more minutely. Is it true that the offspring start just where the parents did, and that they produce just what the parents did? Or is there a gradual change as generations pass, so that evolution occurs?

If the progeny begin just where the parents did and develop in the same way, then if we begin with a single parent and obtain from it great numbers of progeny in successive generations, we shall find that they are the same at the end as they were at the beginning; and that all are alike (save in so far environmental differences have made diversities). There will be no opportunity for some to be preserved because they are better fitted to live, while others die because ill fitted. Is this the situation, or do inherited variations come on as generations pass? 
This problem of the origin of inherited variations, and of the nature and grounds of evolution, meets us in these animals in a peculiarly simple form. For in their reproduction from a single parent it is not complicated by the continual mixing of diverse lines of descent, which enormously confuses the matter in higher organisms. In creatures in which reproduction is always from two parents, the descent of generations takes the form of a network, such as illustrated at $\mathbf{B}$ in Figure 5. If we attempt to trace back the ancestry of any one of the individuals indicated by the lines at the right, we find that it is a mixture of many lines of descent, with diverse hereditary characters; in any given past generation many ancestors of the present stock appear. It becomes extremely difficult or impossible to predict what hereditary characters it should show, or whence it has derived those that do appear; and it is hardly possible to distinguish an actually new character from one resulting from the mixture of earlier stocks. In the Protozoa while descent from a single parent is in progress, the passage of generations takes the form indicated at $\mathrm{A}$ in Figure 5 . There is no mixing of diverse lines, and in any past generation there is but one ancestor for the existing stock; descent can be traced backward in a single line. On account of this relatively simple state of affairs, the origin of variations and the course of evolution has been much studied in the Protozoa. We will illustrate the conditions found by means of a series of figures of Difflugia corona (Figures 19-21).

When we examine a single species, be it a bacterium, a rhizopod (as Difflugia corona) or a ciliate infusorian (as Paramecium aurelia), we find a great diversity in the individuals, in their form, their structure (Figure 19), and their physiology. Part of this diversity is, in some of these creatures, due to the different conditions under which they are living. But if we bring them all into the same conditions 

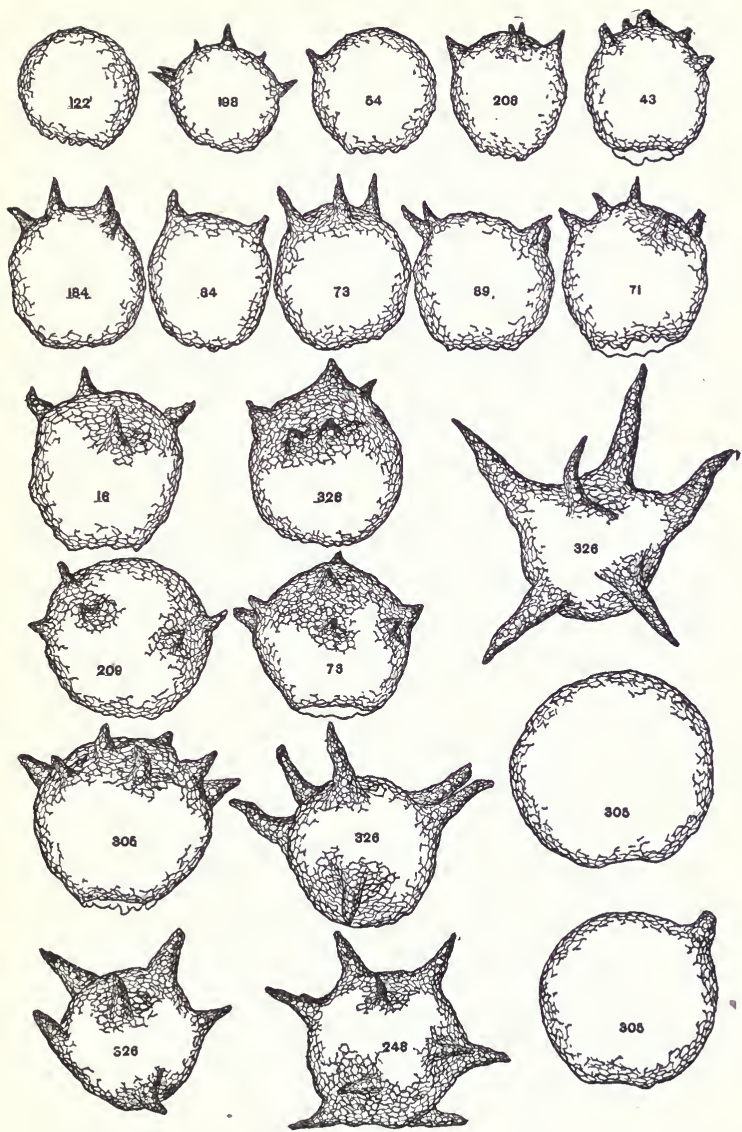

Figure 19. Difflugia corona; collection of individuals to show the variations in size and form; in number, length and shape of the spines, and the like. All drawn to the same scale. (The numbers are the designations of the families to which the individuals belonged.) After Jennings, 1916. 

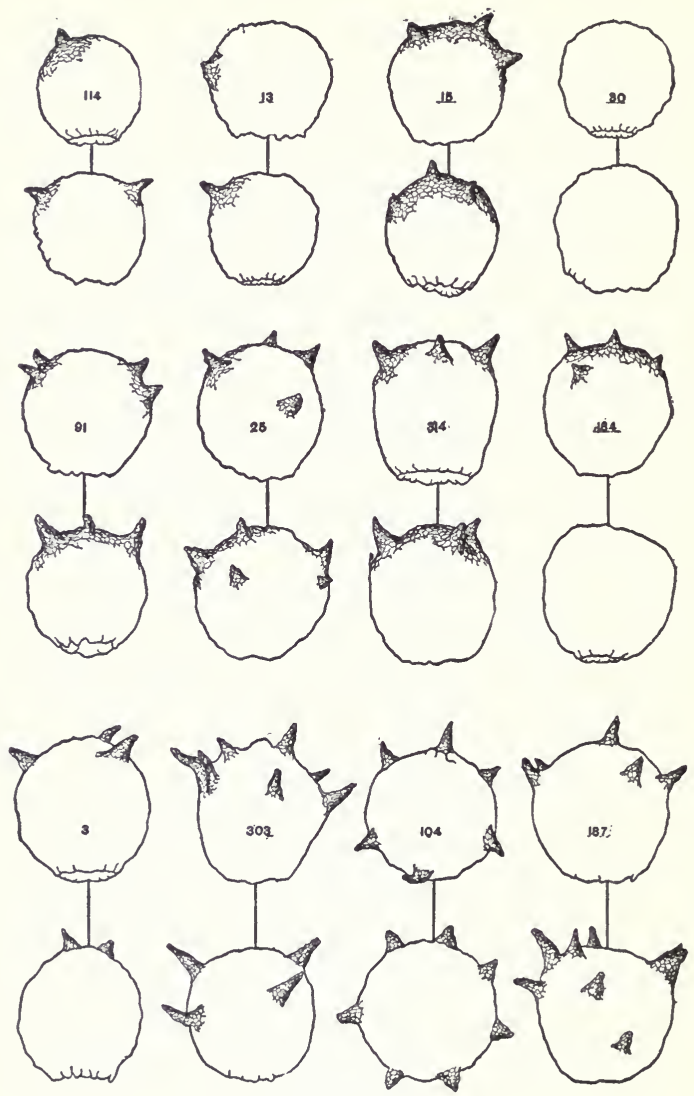

Fig. 20a. 

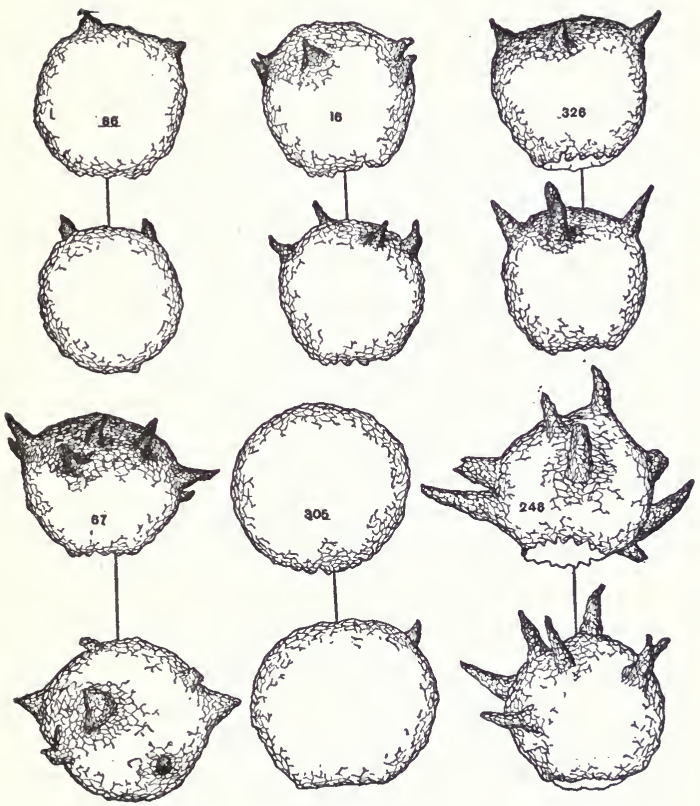

FIG. $20 b$.

Figure 20. Difflugia corona. Parent and immediate offspring in 18 diverse families, all drawn to the same scale, to show the variation and the inheritance of the parental characteristics by the progeny. In each pair the parent is above, its offspring below, the two connected by a line. If each family is bred for many generations, it continues to remain true to the type shown. (The numbers are the designations of the families to which the individuals belonged.) After Jennings, 1916. 
and allow them to reproduce, we find that the offspring repeat in large measure the peculiarities of their parents (Figure 20). That is, the particular characteristics of the parents are inherited,-quite independently of diversity in conditions. If we allow each of the diverse parents to reproduce for generation after generation, we find that in each case the peculiarities of the original stock are retained (Figure 21). That is, each single species, such as this one of Difflugia corona, consists of a large number of hereditarily diverse strains or families; of strains remaining diverse for generation after generation.

This is one of the facts of capital importance in the biology of these organisms; something that has to be kept continuously in mind in all attempts to work with them or to understand them. It forms the key and explanation for many remarkable phenomena in their lives. We shall therefore look at the concrete facts for a number of typical cases, and examine their results in relation to heredity and variation.

In Difflugia corona the number of hereditarily diverse strains that have been found is indefinitely great. They differ in size and form, in the number of spines, in the length of the spines, in the number of teeth surrounding the mouth. Different strains have hereditarily different combinations of these characters; some have large shells with few spines; others have large shells with many spines, and so on for other combinations. -

Besides these marked structural peculiarities, the strains of Difflugia differ in many other ways not apparent to the eye. Some of the strains multiply rapidly, others slowly. Some are very hardy and easily cultivated in the laboratory; others are delicate, dying out under artificial conditions. Some are very active, others quiet. Some are adapted to 

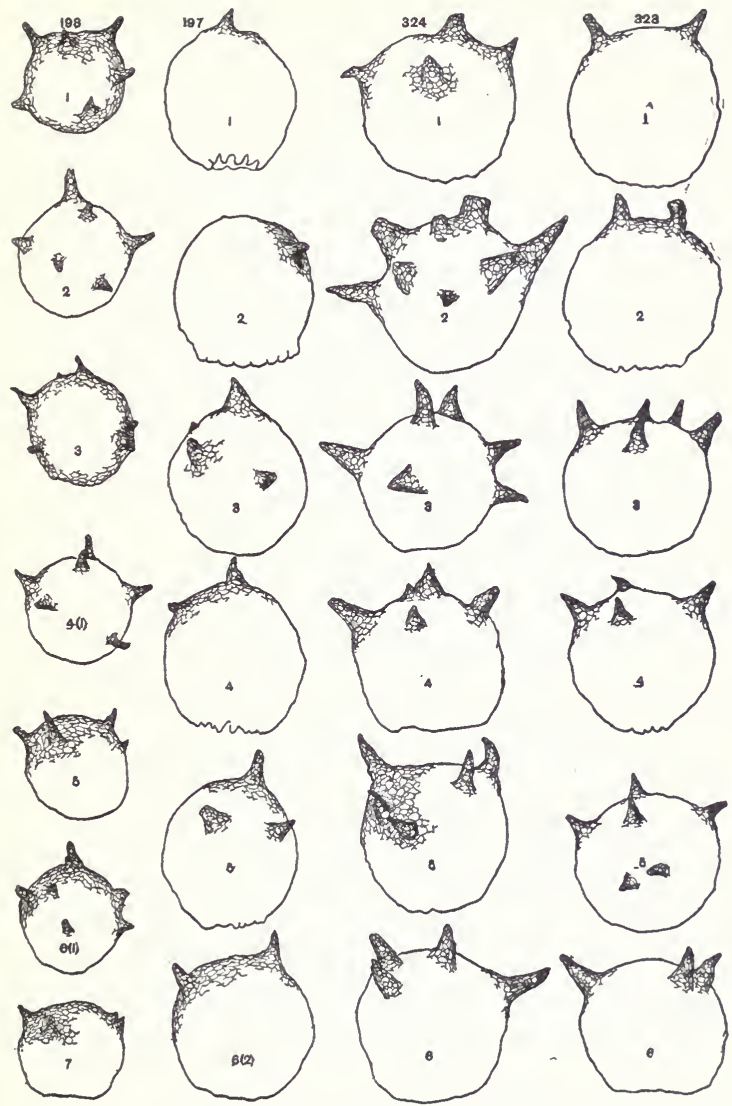

Figure 21. Difflugia corona; portions of four families, to show the inheritance of the diverse combinations of characters. All individuals of any column are descendants of the one at the top. The numbers at the top are the designations of the different families. Observe that in family 198 all are small, but with numerous spines; in 197 , they are larger, but with few spines. In 324 the individuals are large, with large spines; in 323 they are of about the same size, but with small spines. The families remain true to such peculiarities, no matter how long they are bred. After Jennings, 1916. 
one set of conditions, others to other conditions. The existence of these strains presents an enormous diversity within the species; anything that we learn about one strain cannot be transferred directly to another. A similar condition of affairs has since been found to occur in other Rhizopods. Root, $^{1}$ (1918) shows that many diverse stocks exist in Centropyxis aculeata; and Hegner, ${ }^{2}$ (1918) shows the same to be true in Arcella dentata.

In many other Protozoa most of the differences between the stocks are in respect to the characters not readily detectible by the eye. In Paramecium caudatum, or Paramecium aurelia, for example, the visible differences between the strains are mainly in respect to size, and since the size is changed greatly during growth, it requires thorough study to detect the differences of strain. But such study shows that strains of different size do exist (Figure 22). Within each strain there is great variation of size among the different individuals, owing to differences of growth and of environment. But each strain or family has its own characteristic average size. If we pick out any individual of a given strain and allow it to produce many off spring, we shall find that their average size will correspond to that of the family from which they came; an individual from a larger strain will produce larger progeny; one from a smaller strain will produce smaller progeny. Thus a "population" of Paramecium as we find it in nature is made up in the way shown in Figure 22. There are many strains, diverse in mean size, but each with many individuals of diverse size.

In Paramecium, as in Difflugia, these strains differ in other respects also. I found that some multiply rapidly, others slowly; that some conjugate frequently, others rarely.

${ }^{1}$ Root, F. M., Genetics, March, 1918.

' Hegner, R. W., Proceedings of National Academy, September, 1918. 


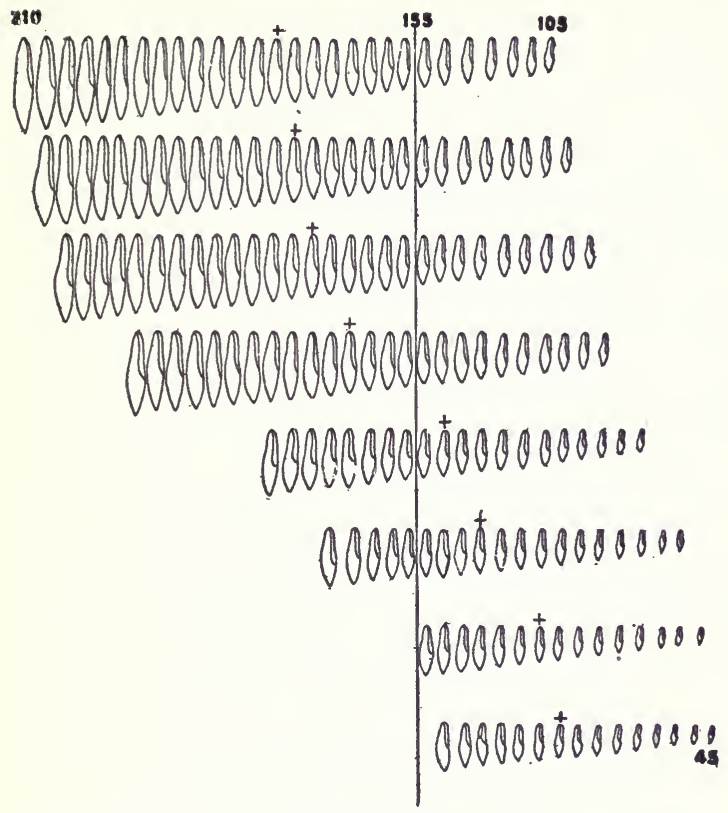

Figure 22. Eight diverse families of Paramecium, showing variations. Each row represents a single family, showing the maximum, minimum and intermediate sizes of individuals of the given family. The differences in size within the family are due to differences in growth and environment. The differences in average size between the families are hereditary.

The numbers show the lengths in microns. The mean length for the entire set together is given by the perpendicular line at 155 microns. The mean size for each family is that of the individual above which is placed a + sign. After Jennings, 1909.

Hutchison (1913) found that they differ in their resistance to heat,- - some strains standing a higher temperature than others; the same thing was observed by Jollos (1913). 
Jollos observed also that some strains are more resistant to poisons than others. Hance (1917) found that a certain race of Paramecium caudatum has a tendency to produce one to three extra contractile vacuoles, so that its members may have three to five of these, in place of the two found in most races. Powers and Mitchell (1910) found a race of Paramecium that had several micronuclei, in place of the single one, or the pair, commonly found.

All together, it is clear that the different races of Paramecium present the greatest diversities in all sorts of structural, and particularly physiological, characters,-so that from our knowledge of the biology of one race we cannot be certain as to that of the others.

A similar condition of affairs is known to exist in many species of bacteria. Diverse families exist, differing in their nutritive peculiarities, in their resistance to chemical agents, in their virulence as producers of disease; in the way they grow on artificial media, and the like.

The condition of affairs has been found to exist in all the Protista that have been thoroughly studied from this point of view. It is probable that it will be found in all species; certainly it would be of much interest to examine thoroughly any species that seems to be uniform, in order to discover if there is such a thing as a species that does not consist of hereditarily diverse stocks.

The same condition of things is likewise found in higher organisms. It is worth while to recall the name and work of the man who first recognized that the so-called species of animals and plants are really made up of a great number of hereditarily diverse stocks, which remain quite distinct so long as they are reproduced without crossing; for this work was done long ago, and, like the work of Mendel, remained for many years quite without influence on the world. In 
recent years it has been rediscovered, and some believe that it is almost worthy to rank with the great work of Mendel, whose obscure fate it long shared. The discoverer, Alexis Jordan, was, like Mendel, a devout Catholic; and was guided in his experimentation (unlike Mendel, apparently) by his theological beliefs. He did not believe in the variability of organisms, as taught by many prevailing doctrines, but maintained that the differences within a species that were commonly cited as variations were in reality permanent differences between races. So as early as 1854 he undertook the culture in his garden of certain common plants, notably Draba verna, the common little weed called whitlow grass. In ten years he was able to show that this contained ten diverse races; after twenty years' culture he announced that he had now found 53 races; and after culture for thirty years he could show that there were 200 permanently diverse stocks of Draba verna. He discovered the same thing to be true for a number of other plants, and maintained therefore that his faith had been verified; the differences found within a species were not variations in the sense of actual changes which occurred, but merely permanent diversities, which Jordan believed had existed ever since the organisms were created; instead of variations that occurred, there was multiplicity that existed. ${ }^{1}$

Now whatever we may think of Jordan's line of argument, the facts which he set forth have been confirmed in recent years for a great number of organisms. And his direct conclusion from those facts likewise stands fast. The differences that we observe among the members of a species are in the overwhelming majority of cases not "variations" in the sense of being due to recent actual changes in the hered-

${ }^{1}$ The facts as to the work of Jordan are taken mainly from Lotsy, 1916 and $1916 a$. 
itary constitution. On the contrary they are merely lasting hereditary diversities between stocks whose origin has not been observed. The situation is very greatly confused when these diverse stocks continually intercross, as happens in so many higher organisms, but it is not thereby essentially changed.

The situation in these lower organisms is very much what it would be in man if in man new individuals were regularly produced by the division of those already existing. We have reason to believe that this practically does occur in the case of identical twins; they are produced by the division of a single egg, which if it had not divided would have produced but one individual (see Newman, 1917). If this occurred in man regularly and frequently, as it does in most Protozoa, we should find that the human population contained great numbers of individuals as precisely alike as are identical twins. Each of us would meet his precise counterpart at every turn. All these closely similar individuals of one type, taken together, would correspond to a single one of the stocks or races of the Protozoa. And as in the Protozoa, there would exist great numbers of such diverse stocks; in man as many as there now exist hereditarily different individuals. That is, each person present in this room would represent a diverse stock or race; for each person has a constitution hereditarily diverse from every other (save in the case of identical twins).

Now this fact that a species consists of a great number of hereditarily diverse stocks or races, often differing in only minute particulars, throws a most unexpected, and to many persons unwelcome, flood of light on many supposed studies of evolution, and particularly on the effects of selection in the breeding of organisms,- - giving to such studies a significance quite diverse from that which they 
were supposed to have. A great school of biologists, the immediate followers of Darwin, forming the so called English biometrical school, set themselves the problem of measuring variation, inheritance, the effects of selection, and from these the rate of evolution. In so doing they assumed these existing differences as variations, and based their calculations upon these; they found of course a high degree of inheritance from diverse parents, and they found that by selection rapid progress could be made in changing the species. But if we examine a species made up of a lot of hereditarily diverse strains (for example, Difflugia corona), it is evidently easy by selection of a particular character to obtain a set of animals that differ from the average in that respect; one merely picks out representatives of the races that have the character for which we are selecting. Thus, in Difflugia corona, if one desires to increase the average numbers of spines, he will pick out parents with many spines. These belong to races in which a large number of spines is hereditary, so that after selection the progeny produced will have a higher number of spines than was the average for the species before selection. By continuing the process of selection, we gradually exclude more and more completely the races with few spines, and so by selection we make steady progress in increasing the number of spines in this animal. Similar results follow from selecting for any other inherited character; and in any species composed of diverse races, the same sort of results are reached.

Thus in a population of Paramecium we can easily obtain by selection all sorts of apparent hereditary alterations in size; in nutritive peculiarities; in resistance to heat or to chemicals; in reproduction; in all sorts of characters. All these and many other results have been produced again and again, in this and in other species. But what we really do 
is to pick out races that already have these peculiarities. This is unquestionably the explanation of the effects of selection in by far the greater number of experiments or observations where it is found to have an effect.

Such results were long interpreted as showing actual steps in evolution; by selection changes in hereditary characters were thought to be produced, and new hereditary characters obtained. On the basis of such interpretations the rate of change through selection, the rate of evolution, was measured.

But what really occurs in all such cases is a gradual picking out of existing races with certain characteristics (in our example, with numerous spines), and discarding the rest. So far as the selection is based on these differences between preexisting stocks, no evolutionary change has been produced or measured.

But can selection do nothing more than this? Are there no actual changes in hereditary characters? Are these diverse races really permanent in their hereditary characters? Or do changes gradually occur even within such stocks? How do these diverse races happen to exist? Can several diverse races be produced from a single one?

On this question a great deal of work has been done in the unicellular forms; we shall examine the results.

One of the animals most studied from this point of view is Paramecium. If we take a wild set of these animals, it is easy, as we have seen, to bring about differences by selection, for all we have to do is to pick out such as we please of the different races that exist. But suppose we take but a single race; suppose we begin with a single individual and get our entire population from this one parent. Shall we then be able by selection to bring about hereditary differences? In other words, do any hereditary changes occur in such a single race? This question is evidently the fundamental one 
for evolution, for if no such changes occur, there would appear to be no such thing as evolution.

I made long continued attempts to change the size in such a race of Paramecium, by selecting on the one hand large individuals; on the other hand small ones (Jennings, 1908). No effect was produced. Large parents and small parents, if they belonged to the same race, produced progeny of the same size. Ackert (1916) has recently repeated these experiments, with the same results.

I tried also to produce from a single race stocks differing in their rate of reproduction, but these attempts, like those to change the size, met with no success. Each race seemed permanent in its size and rate of reproduction.

Jollos (1913) attempted by selection in Paramecium to obtain stocks that differed in their resistance to heat and to chemicals. He found this very easy when he began with a wild population containing many diverse stocks; all he had to do was to isolate the differing stocks. But when he began with a single stock, he found that he could not by selection get stocks diverse in their resistance. Throughout the ordinary multiplication, all the individuals, like identical twins, remained just alike.

Similar results have followed many other attempts to change by selection the inherited characteristics of a singie stock. Barber (1908) made long continued attempts to change in this way the characteristics of pure stocks of bacteria and of yeasts. He found a very few cases of sudden mutation, such as occur with similar rarity in higher organisms; of these we shall speak later. But, as a rule, any differences found within a single race were not inherited; the races were permanent. Wolf (1909) made repeated and long continued attempts to change through selection the colors in pure races of the red bacterium, Bacillus prodigiosus; but he could produce no change in this way. 
Thus in general, until very recently at least, the experience of investigators has been such as to confirm what was said many years ago by that greatest of investigators of the Protozoa, Émile Maupas. In 1888 Maupas, after long continued study of Protozoa, said:

"In long and numerous experiments on fifteen to twenty species, I have never observed anything which permits belief in the existence of morphological and physiological differences between, not merely the products of a given fission, but even among all those which have descended from such a fission by regular and continuous generations." 2

The same sort of results have been reached from the study of higher organisms when they reproduce without mixture,- - the progeny arising from a single parent instead of two. The most famous work of this sort is the study of self-fertilizing beans, made by Johannsen (1903). Diverse races existed, but in seven years of selection no effect was produced on the characters studied, so long as the selection occurred within the limits of a single race. Lashley (1915) studied Hydra in the same way; no effect was produced from selection continued for many generations. Ewing (1916) attempted to modify by selection plant lice multiplying parthenogenetically. The work extended over eighty-seven generations, and many different characters were investigated for longer or shorter periods. In no case could he change the hereditary characteristics by selection. Agar (1914) studied in a similar manner certain of the lower crustacea multiplying by parthenogenesis, and reached the same results. A great number of investigations could be cited, all ending in the same way. The organisms studied contained many diverse races. But when a single race or "line" is studied, not mixing with other races, the differences between

${ }^{2}$ Maupas, 1888, p. 176. 
individuals were not inherited, and long continued selection was without effect. This sort of study has come to be known as the "pure line" work, and the general result of it all has been that "selection has no effect within a pure line."

Such results have profoundly modified the theories of heredity and of evolution. The Danish investigator Johannsen (1913) has based on them and similar results a general system or doctrine of heredity. According to Johannsen's views, the hereditary constitution of a given organism is a perfectly definite thing, not subject to gradual or indefinite fluctuation. This constant hereditary constitution he calls the genotype. The genotype is comparable to a definite chemical compound. It may become altered sometimes, as a definite chemical compound may by certain reactions be transformed into another and diverse compound; such complete transformations are called mutations; they are extremely rare. The genotype is not subject to slight and gradual alterations, any more than is the nature of the chemical compound $\mathrm{NaCl}$. When diverse genotypes are mingled, as in reproduction from two parents, the results give us what is called Mendelian inheritance. We cannot take up the details of this here, but the same principles hold. Selection in such cases, according to this view, merely brings about varied combinations of things already existing; the nature of its effects is therefore the same as when it is applied to organisms descending from but a single parent. This view of the matter may perhaps be said to have dominated recent work in heredity.

This constancy of races in organisms descending from a single parent, and the application, which appears unavoidable, of the same point of view to the cases where there are two parents, presents very great difficulties for the theory 
of evolution. It could indeed be plausibly maintained that all these results are opposed to the theory of evolution; that the logical conclusion to be drawn is that which was maintained by Jordan, the originator of this sort of worknamely, that there is no variation; no change from generation to generation; no evolution.

Is that indeed the conclusion to which we are driven? Is that the upshot of the modern attempts to study evolution experimentally; to see evolution occur? We shall take up this question in our next chapter. 


\section{III}

Results of Intense and Long Continued Study of Changes in a Stock. Inherited Variations in the Pure Race. Visible Evolution.

I $\mathrm{N}$ our last chapter I tried to picture the first results of the attempts to study evolution experimentally-to actually see evolution occurring-to see variations take place and to see their inheritance.

These first results were:

That any kind of organism is really composed of a great number of diverse stocks or races, whose differences are hereditary, lasting from generation to generation;

That the supposed effect of selection in modifying organisms consists in isolating certain pre-existing races, having the characteristics that one is selecting; or in making, through biparental inheritance, new combinations of characters that already exist;

That in general, the apparent variations in organisms are not real changes in their hereditary constitution, but are merely these static diversities persisting from generation to generation;

That when one takes a single one of these races and tries to discover in it hereditary variations, or to modify it by selection, he finds it extraordinarily constant, and his efforts are without result. That seemingly the only variations which appear are sudden large mutations; that gradual alterations do not show themselves. 
I wish to emphasize that this is a picture of the present situation of affairs; that it gives the prevailing theory of these matters. If you will read the address of President Pearl of the American Society of Naturalists, published in the American Naturalist (Feb. 1917), you will find that this is the theory which he presents. I have no doubt that if there are any experimental workers in heredity in this audience, this is the theory which they maintain.

Now, as I remarked at the end of the last chapter, this situation of affairs presents great difficulties for the theory of evolution,-leading indeed logically to the conclusion that evolution does not occur. And even when we add to these results the observed cases of sudden change of characters, which are called mutations, it becomes extremely difficult to see how evolution can occur. For most if not all of these mutations, as is well known, consist in defects and losses; and it is difficult to believe that evolution has occurred by repeated losses,-although attempts have been made to maintain even that paradoxical theory (Bateson, 1914; Davenport, 1916).

Moreover, there are certain facts about organisms which it seems impossible to explain by the appearance of sudden extensive mutations. In the organisms that we have been describing we find that the hereditary differences between the races are as minute as can possibly be detected by the most refined methods; they run down to the very limits of visibility with the microscope. Such are the differences between the diverse races of Paramecium (Figure 22); such those between the races of Difflugia (Figures 19 to 21). It is clear that such differences cannot have been produced by "saltations"- by mutations of large extent. And the same condition of affairs is found in higher organisms; the differences between Jordan's 200 races of Draba verna were so 
slight that it took years to detect them with certainty. If such minutely differing races have been produced from a single one, the steps in the change have been at least as minute as these differences. If evolution really occurs, why should we not see these minute changes?

Again, the existence of complex adaptive structures, such as the eye or the ear, presents difficulties for the theory of origin by extensive mutations perhaps fully as great as does the existence of races differing only by minute grada. tions. The difficulty here is not so readily presented in a simple way, but to me it appears that thorough analysis would reveal it as an insuperable one.

Furthermore, paleontologists maintain, with practical unanimity, that the study of extinct organisms shows that the change in the characteristics of animals of a given stock, as we pass from one geological period to another, has not been by leaps, but by gradual alterations. The study of paleontology is the most direct study possible of past evolution; we cannot neglect its conclusions.

On the whole then it is difficult to rest content with the results of what we may call this first examination of the diversities in such organisms. Shall we yield to the argument of Jordan, that evolution is not occurring? Or shal we rather proceed to more refined studies; to what might be called investigations in the second degree? In our first studies have we not possibly been overwhelmed and halted by the great discovery that most of what we had thought were real variations and real effects of selection were deceptive,-were mere consequences of the existence of hereditarily diverse races,-so that we have stopped before the end? Shall we not next merely accept all this that has been learned in the last fifteen years as a background, take a new hold; select the most favorable organism possible; a void 
all sources of confusion met in the earlier studies, limit ourselves to one single race, pursue its history with more minute and unwearying steadiness, for longer periods than has before been done,- to such a degree that we may properly call our studies investigations in the second degree,-as compared with the earlier ones?

This is what I decided four years ago to attempt; with a number of associates to set on foot such "second degree" investigations. They have now been carried far enough to show results. The main difficulties in the previous work along these lines have been the following:

(1) In these simple organisms it is difficult to find definite distinctive characters, such as color of eyes or hair, that are inherited. In Paramecium, for example, all the individuals are very similar, the diversities being mainly slight differences in size or shape; or in indefinite physiological traits. Such characters are not favorable for work in heredity because they are hard to distinguish; yet in practically all the earlier work such characters were employed,for the good reason that these seemed the only characters available.

(2) Further, the characters studied have been such as were continually changed by growth during the life of the animals; and likewise greatly modified by changes in environmental conditions.

The first thing to do therefore was to find if possible an organism in which these difficulties did not exist. From this point of view an ideal animal was found in Difflugia corona, - the creature that I have already employed in these lectures to illustrate a number of points. This animal is an amœba with a shell, and the shell presents a number of definite characters that can be easily counted and measured (Figure 23). These characters are: the number of spines; 


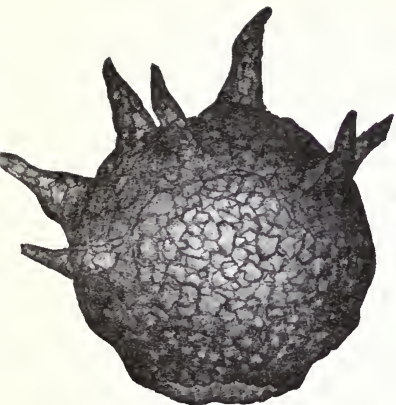

A

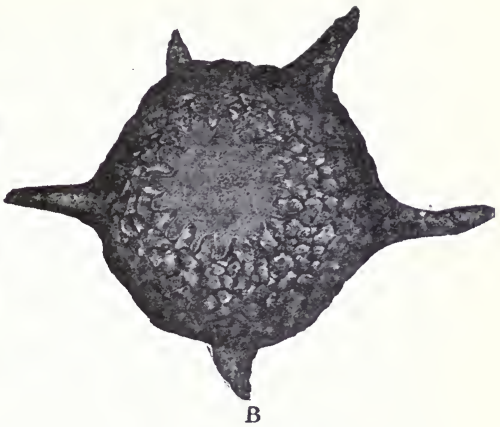

Figure 23. Difflugia corona, to show the characters studied in the work on inheritance. A. Side view. B. Oral view, showing the mouth and the teeth surrounding it. After Jennings, 1916.

the length of the spines; the number of the teeth about the mouth (Figure 23, B); and the size of the shell, as measured by its diameter. All these characters are formed when the individual is produced (Figures 11 and 12), and are not subject to change by growth, nor are they altered by changes in the environment during the life of the individual. No more favorable combination of characters for the study of variation and heredity could possibly be found.

Difflugia is extremely fastidious as to just how it shall live and what sort of food it shall be furnished, so that it is not easy to keep pedigreed stock for generation after generation, as is necessary for all work in heredity. When these difficulties are overcome, we follow for long periods the history of a given race. As we have seen, when we compare different races, the peculiarities of the parents reappear in the offspring in a high degree (Figures 19 to 21). But diversities do arise even within a single race. Parents with many spines have as a rule progeny with many spines, but 
often the number is not the same in the progeny, and the successive progeny of the same parent may have different numbers of spines. As we pass from parent to off spring, similar variations arise as to length of spines, number of teeth, and size of the shell. These facts are illustrated in Figures 19 and 20.

Now the question of interest is, whether these differences within the single race (all derived by fission from a single parent) show any tendency to be inherited. When a single parent produces one offspring with few spines and another with many spines, does the former tend then to produce a set of progeny with few spines, the latter a set with many? If so, we have the beginning of the origin of two races from one. Or will there be mere chance variations, -with no tendency on the part of the later descendant to reproduce its parent's peculiarities?

Study shows that there is certainly no complete or even very marked tendency for the progeny within a race to reproduce the diverse peculiarities of the parents. If a parent has many spines, some of its offspring have many, some few ; if the parent has few spines, some of its offspring have many spines, some few ; and so of all the other characters (see Figures 20 and 21). To get any results whose meaning is clear, we have to resort to averages, and to mathematical measures of correspondence, for very large numbers of parents and progeny belonging to a single race. What we have to do is to determine whether, within a single stock, on the average and in the long run, parents with many spines produce offspring with a greater number of spines than do parents with few spines.

When we do this we find in Difflugia indications that there is some correspondence between parents and progeny. In some cases the indications are very slight; in others more 
marked. Measuring by the coefficient of correlation, we find that when the parent differs from the average, the progeny tend to inherit somewhere from one-tenth to three-tenths of this peculiarity. That is, the race shows a slight tendency to break up into several races, hereditarily distinct. (For details see Jennings, 1916.)

For the investigator who has searched in vain for years to find in uniparental reproduction any tendency for a single race to evolve into several, such faint indications are exciting; here we begin to get hold of the beginnings of evolution. Most of the grounds on which we believe that evolution occurs are inferential; we believe that it must have occurred, -in order to account for the diversities that we find now existing. But can we actually see it occur?

To carry our work farther, we begin to exercise selection within the single family. On the one hand we select all the long-spined individuals and place them together; on the other hand we select all the short-spined ones and place them together. In the long-spined group we continue to save for generation after generation only the individuals that are long-spined; in the short-spined group only the off spring with short spines. In the same way we select other sets for numerous spines and for few spines; for large shells and for small shells; for many teeth and for fewer teeth.

And now as we keep this up for generation after generation we find that the correspondence between parent and progeny becomes more and more marked. We find that our single family is breaking up into many different groups, which differ from one another hereditarily. We get finally what appear to be two diverse races,- - one with long spines, the other with short spines (Figures 24, M and F), the difference continuing for generation after generation. A third set (L) has constantly large shells, while others 

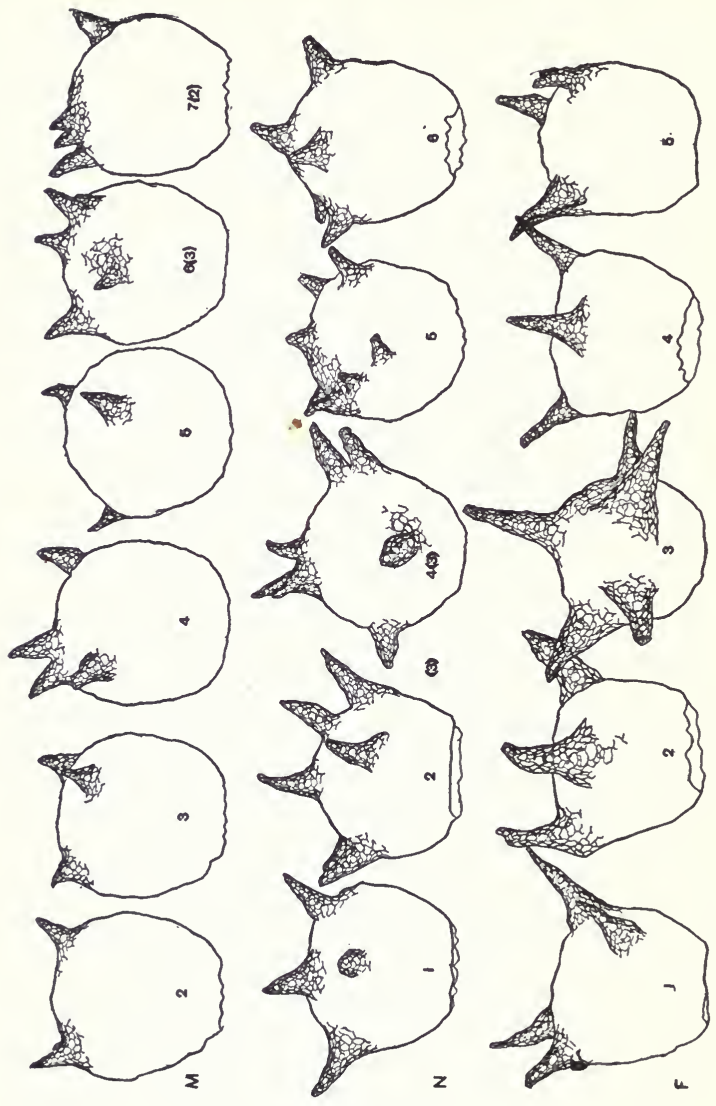

Frg. 24A 


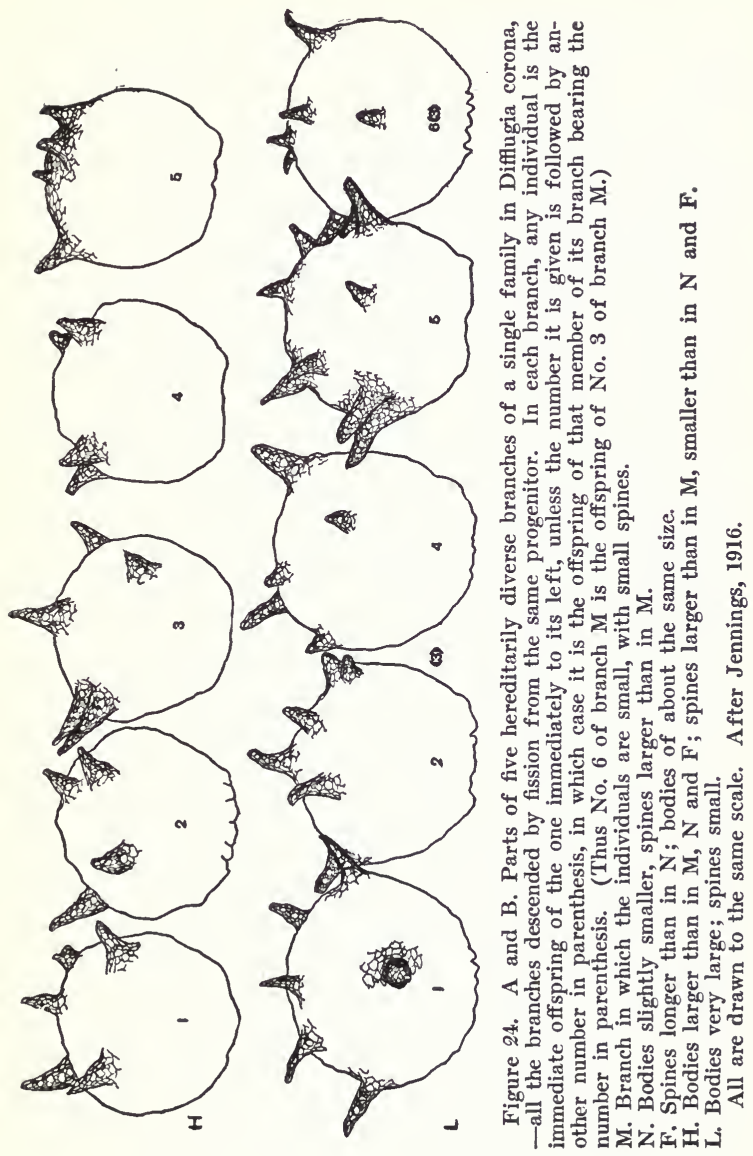


( $M$ and $N$ ) consistently produce small shells. We also get stocks hereditarily different for numbers of spines; and for numbers of teeth. Our single stock, derived by fission from a single parent, has gradually diversified itself into many stocks that are hereditarily different. If this is what we mean by evolution, we have here seen evolution occur.

In Figure 24 we see a number of the hereditarily divérse stocks that arose by fission from a single parent, in one of my experiments. It will be worth while to summarize the main facts as to the appearance of hereditary variations in this animal.

(1) Hereditary variations arose in some few cases by rather large steps, which might be called mutations, or saltations.

(2) But the immense majority of the hereditary variations were minute gradations. Variations, are as continuous as can be detected.

(3) Hereditary variations occurred in many different ways, on many diverse characters: the number of spines; the length of the spines; the size of the body; the number of teeth. There was no single line of variation that was followed exclusively, or by the great majority of cases.

(4) Any set of characters gave rise to variations independently of the other characters. Thus many diverse combinations of characters arose; large animals with long spines; small animals with long spines; large animals with short spines; short animals with short spines, and so on for other sorts of combinations.

(5) The hereditary variations which arose were of just such a nature as to produce from a single strain the hereditarily different strains that are found in nature.

I judge that if the intermediate strains were killed off, the two most diverse strains found in nature might well be 
classed as different species,- - although the question of what constitutes a species must be left to the judgment or fancy of the individual.

Since the work on Difflugia was done, the same methods of work have been applied in our laboratory to two of its relatives, Centropyxis aculeata and Arcella dentata. In the former, Root (1918) found considerable evidence that certain variations within the single stock were inherited, although the work was not carried so far as in Difflugia. In Arcella, Hegner (1918) found that heritably diverse stocks could be isolated by selection from a single stock multiplying by fission.

All together then, our "second degree" study of the matter has been rewarded by the discovery that for these animals at least the situation that I sketched in my last lecture is not final. In these animals we do find the diverse races present under natural conditions, just as in other organisms (see Figures 20 and 21); and by mere selection among these diverse races we can get all sorts of apparent changeswhich are not real changes; which are not evolution. But when we take a single race and devote all our attention to that alone for years, then we find that real changes do occur; that the race differentiates into many races in the way I have described; that evolution visibly does occur.

Now I told you that the other theory was the prevailing one; so much is this the case that some of my readers will not accept unreservedly these cases as actual changes in hereditary constitution; as actual steps in evolution; on the contrary they are trying to devise various possible schemes by which it could be made to seem that even here in Difflugia we are getting nothing but new combinations of what was before present. Many such schemes have been devised for explaining apparent effects of selection in higher 
organisms. None of them can be applied directly to Difflugia, since here we have uniparental reproduction, and most of these schemes depend upon the mixing of two stocks. But other schemes can be devised, which might apply to Difflugia; we shall mention some of these. At present I wish to ask your patience for a few moments for a closer analysis of just what has happened in such a case as this. Such an analysis will bring out the main questions and difficulties that can be raised.

What then is it that has actually occurred in such a case? We began with a single individual; it consisted of a shell filled with a mass of protoplasm, containing one or more nuclear bodies. This mass had behaved in such a way as to produce a shell of definite size, form, number of spines, and the like. We found that when this mass of protoplasm gives off one-half of itself to the outside of the old shell, this half is made up,-chemically or otherwise,-in the same way as was the original parent mass; for it does just the same things that the parent mass did. That is, it produces a shell essentiailly like that of the parent,- of a similar size, shape, number of spines, and the like (see Figure 12). This is particularly striking when we compare this individual with others of different race, or of different species, as in Figure 20 ; it is extraordinary to see these tiny masses of protoplasm, each conducting itself in a manner different from any other; each holding true to type. There must be very definite, and at the same time very delicate, chemical differences between them.

But as we follow for a long time our original individual and its progeny, we find that the chemical nature of the protoplasm very gradually changes as divisions occur, for the behavior begins to slightly change. Although all under the same conditions, some of the masses commence to produce 
longer spines; others shorter; others more numerous spines; others fewer (Figure 24). Different masses change in different ways; the number of kinds of diversity that we get is large; apparently indefinitely large. The protoplasm certainly gradually becomes diversified as it continues to exist and increase.

A number of important questions at once arise. What part of the protoplasm is it that thus changes? Is it the cytoplasm, or the nucleus, or is it both? And how does the change occur? Through irregularities in the division of certain substances or parts? Or through chemical or physical changes produced by the environment,- - by changes in temperature, chemical composition of the water, by food, or the like? And why do we find these changes to occur in Difflugia when we could not find them in Paramecium; when in almost all the other organisms studied in this way such changes have not been discovered?

All these questions are bound up together. We shall perhaps deal best with them by taking up the last question first. Why do we find such a difference in this respect between Difflugia and other organisms,-for example, Paramecium?

As was set forth earlier, Difflugia was selected for study precisely because it was much more favorable for such work than Paramecium or most other organisms investigated. In Difflugia there are many well defined distinctive characters, which are not modified by growth, nor changed by the conditions under which the animals live. In Paramecium and most other forms studied, on the other hand, the characters are continually changing through growth and environmental action. Such changes are well known not to be inherited. This makes a tremendous difference as to accomplishing anything by selecting any particular character. Thus in 
Figure 22, showing the diverse races of Paramecium, we see that in any single race there are individuals of many different sizes. But almost all these differences are matters of age, nutrition, and the like. So when we select and separate large and small individuals, we are likely to get merely wellgrown, well-fed individuals in one set; young, ill-nourished ones in the other. Even if there are arising really hereditary differences in size, we cannot distinguish these from the much more numerous transitory changes, so that our process of selection may be rendered quite without hereditary effect. Similar difficulties beset any attempt to select for other characters that are dependent on growth and present environmental conditions.

It appears possible therefore that the difference between the results of selection in Difflugia and in other organisms is due to these facts; that there is no real difference as to the sort of thing that happens, but only as to whether one can detect the hereditary changes that actually occur. Are we to believe that the hereditary constitution of parent and progeny is actually identical in these other forms? Or shall we find changes in it, if we study with sufficient minuteness?

Now on this point we have a certain amount of evidence, based again on what I have ventured to call our "second degree" investigation of these matters. If we watch the divisions of Paramecium or of any of its relatives, we find that the two individuals produced by the division of one do not always behave exactly alike; after they have grown to adult size, one of them often divides before the other does (see Figure 25). Are such differences due to some change in the fundamental and hereditary constitution, or only to some slight difference in nutrition or the like? Here was an opportunity for minute study of the matter; it was undertaken in our laboratory by Middleton (1915). $\mathrm{He}$ 
investigated from this point of view the infusorian Stylonychia. Beginning with a single individual, he selected on the one hand the off spring that divided first; on the other those that divided last. Continuing to select for rapid fission rate in one line, for slow fission rate in another, and keeping this up for hundreds of generations, he found after many generations that there were real inherited differences. Two sets

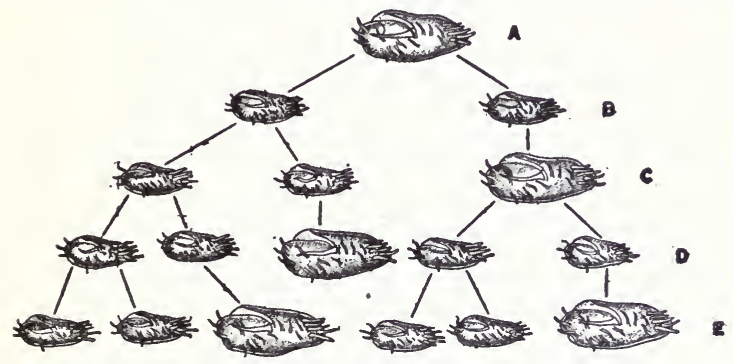

Figure 25. The infusorian Stylonychia; diagram to show the differences in time of fission in offspring of the same parent, with the method of selection for rapid fission and slow fission. Constructed on the basis of a figure of Middleton, 1915.

$A$, the parent divides into two offspring (B), one of which divides into two while the other still remains undivided (C). Similar conditions appear in $\mathrm{D}$ and $\mathrm{E}$. Thus in $\mathrm{E}$, the large individual at the right is the result of but two divisions from $A$, while the small individuals to the left are the result of four.

were produced from among the descendants of a single parent,-one set that divided more rapidly than the other. The difference persisted for long after selection had stopped.

Thus it was shown that in this case hereditary diversities are arising, even with respect to a character so readily modified by the environment as is the fission rate.

This, so far as it goes, of course tends to raise the presumption that other characters of such organisms will be found to show similar hereditary changes when studied with 
sufficient thoroughness,--so that there would be no real difference in this respect between them and Difflugia. I should like to say, however, that from experience with Difflugia and other organisms, and from the work of other investigators, I am personally convinced that there is a difference between organisms as to the frequency with which hereditary variations occur. They occur on the whole relatively frequently in Difflugia. In many other organisms the germinal material is apparently so protected, and so precisely divided at reproduction, that such changes are rare.

And this brings us to the question as to just what in the organism it is that is altered when the hereditary characters change. A number of possibilities are open here. Hegner ${ }^{1}$ (1919) discovered that in Arcella the hereditary size varies with the number of nuclei or the amount of chromatin present, and that these change at times as a result of irregularities in division. The number of spines further was found to be related to the size; larger individuals have more spines than smaller ones. Hence hereditary diversities in the number of spines also were brought about by alterations in the number of nuclei or volume of chromatin. Some of the hereditary changes in the characters of Difflugia may have been brought about in the same way, but it is clear that most of them were not. For as we have seen, some of the new lines produced were small with large spines, some large with large spines,- - the different characters being independent in their hereditary diversities, so as to give stocks with different combinations of characters. These cannot be accounted for by quantitative alterations in the amount of the nuclear material.

Another possibility lies in certain peculiarities of the nucleus in such animals as Difflugia. There is in addition to one or more very sharply defined nuclei a cloud-like mass bf nu${ }^{1}$ Hegner, R. W., Proceedings of National Academy, January, 1919. 
clear material spread through the cytoplasm; this is known as a chromidium. Unfortunately these things are not yet thoroughly known for Difflugia itself, but in some apparently close relatives, such as Arcella (Figure 26), they have been much studied. In the division of these organisms the nuclei divide with the same minute precision that is evident in higher creatures. But the chromidial masses merely separate loosely into halves, with no indication of precision. So possibly the offspring may get different parts of the chromidium in different cases, and it has been suggested that

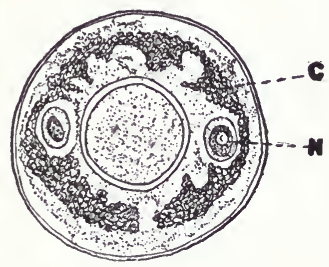

Figure 26. Arcella vulgaris, to show the two nuclei $(N)$, and the chromidium (C), or loose cloud of nuclear material. After Hertwig.

the differences that arise in the hereditary characters are due to this inexactness of division. But this is all speculation as yet, without much foundation of probability.

But in any case it appears to me that these details do not affect the main fact, which is that in these organisms gradual inherited variations are occurring, so that in the course of time many hereditarily diverse families arise from one. In other words, if we study these organisms with sufficient minuteness and perseverance, we see evolution occurring.

We are now in position to sum up the facts as to heredity and variation in these animals when they are reproducing from a single parent. Any species consists of a great num- 
ber of hereditarily diverse families or races, whose characteristics show a high degree of permanence from generation to generation. The offspring inherit in a high degree the characteristics of the parent. But this inheritance is not through an actual handing on of the parent's characteristics; on the contrary the offspring have to produce anew the same kind of characters that the parent had. For this reason any peculiarities acquired by the parent during its life time are not inherited.

Inheritance is very exact, but when we study a family for many generations, we find that it is not absolutely precise, for minute hereditary variations gradually appear, and the single race separates into many hereditarily diverse races. The process of evolution becomes visible. 


\section{IV}

Can We Experimentally Change the Hereditary Characters? Heredity of Environmental Effects. Heredity and Variation in Bacteria and Similar Organisms.

$\mathbf{W}^{\mathrm{E}}$ have seen that in Difflugia hereditary variations arise even when the organisms are all kept as nearly as possible under the same conditions. Thus from a single strain, all derived by fission from one ancestor, many strains arise, diverse in their hereditary characters.

Can such changes be brought about by the action of special conditions in the environment? Can we experimentally produce such hereditary changes? Have some of the diverse strains existing in nature been produced by action of the environment? We saw in our introductory lecture that structural characters produced in the body of Protozoa during the lifetime are not inherited any more directly than are such characters in higher organisms. To be inherited, the acquired structures must be produced anew by the offspring, and for most acquired characters this does not occur; the offspring are produced in the same state that the parents were. But there still remains the question whether the organisms cannot be so altered that in the succeeding generations characters will be produced that are different from those produced in the earlier generations.

\section{Bacteria}

Most of the significant work bearing on this question has been done on the bacteria, and on other organisms that 
have to do with the production of disease. In bacteria the knowledge of variation and heredity has passed through the same series of stages that we noted in other organisms. At first there seemed to be a mere chaos of diverse forms, with no constancy or order; any kind of bacterium seemed producible from any other, or from other organic or inorganic sources. Then came a period of thorough study, with development of precise technical methods. It was discovered that there are a very great number of kinds of bacteria, but that each remains true to its characters; each is produced only by pre-existing individuals of the same race. The differences between the races are often minute, mere matters of a slight diversity in the chemical processes, in the kind of sugar that is fermented by the particular race, or the like. But each race remained true to its type, even in these minute physiological details. This stage of knowledge is the same as that on which is based the theory of the constancy of genotypes in all sorts of organisms, - a theory that we sketched in Lecture 2. The constancy of the races af bacteria has been set forth as one of the facts opposed to the theory that organisms are undergoing evolutionary changes.

But in recent years a still more intensive study has brought to light, in this group as in others, the actual occurrence of hereditary changes, the production, from a given race, of other races whose hereditary characters are diverse from those of the parent race. In the bacteria, more than in any other group of organisms, something has been learned of the conditions which bring about these changes, though knowledge on this point is still scanty.

The bacteria present extreme difficulties for the critical study of heredity and variation, owing to their minuteness. To be certain of what the results mean it is necessary to 
work with races all members of which are derived from a single original individual. It was long impossible to fulfill this requirement; the so-called pure cultures of bacteria were derived from a large number of individuals. There might be slight racial differences between these original individuals, the different strains being adapted to different conditions. Then under given conditions one strain multiplied until the entire population seemed to take on its characteristics, the other strains remaining without activity or multiplication. But on a change of conditions this prevalent strain ceased its multiplication, while some other strain became active, multiplying until the population showed the characteristics of this second strain. It appeared as if the changed environment had altered the hereditary characteristics of the organisms, but this appearance would be illusory. It seems probable that such impurity of the original stock accounts for some of the apparent transformations that have been described.

But in recent years a number of methods have been devised for isolating a single bacterium, so that an entire stock can be derived from this. ${ }^{1}$ From such pure races dependable results can be obtained.

One of the first to isolate pure stocks was Barber (190\%); he worked both with bacteria and with yeasts. Barber did not attempt to modify the organisms, but merely to determine whether the variations in size and form often observed are inherited. From a pure race he picked out large individuals, long individuals, individuals of peculiar form, and ${ }^{1}$ Apparently the simplest and most effective method is that of mixing a very little of the fluid containing bacteria with a large quantity of India ink, then producing a thin layer of this ink between two cover glasses. Single bacteria, owing to the fact that they are surrounded by a cloud of gelatinous material, are visible as minute clear specks in the dark ink. A cover glass preparation containing but a single individual is taken as the beginning of a culture. This is known as Burri's method. 
the like, and determined whether their descendants inherited their peculiarities.

Barber discovered the same thing that others have found in other organisms; in the very great majority_of cases such peculiarities within a race are not inherited. Great numbers of indivduals selected for certain peculiarities gave offspring of the usual types. Nevertheless a few heritable variations were discovered. In Bacillus coli, 140 individuals that were longer than usual were isolated. All but one gave descendants of the usual size, but this one gave a race having bodies longer than usual. The race was permanent; selection of longer and shorter specimens within it was without further effect. Two other long-bodied races were obtained in later extensive selections. Similarly, from among a great number of selections of peculiarly shaped yeast cells, a number of new races were obtained in which the cells were narrow and elongated, as compared with the more nearly spherical cells of the parents.

What caused the production of these new races is not known, but they demonstrate that in bacteria and yeasts at times the inherited characteristics of a race become altered.

More definite results have been reached in the study of color changes in bacteria. The organism known as Bacillus prodigiosus produces a bright red color; it is supposed to be the cause of the "miracles" in which the bread of the host appears to become bloody. Wolf (1909) attempted by various means to obtain from this organism races that give a different color or that are colorless. A series of fifty successive selections of the lightest parts of the colonies produced no inherited effects; the descendants were still of the typical color.

Wolf further tried cultivating the colored bacilli on media containing chemicals of various sorts. He employed in different cases copper sulphate, potassium bichromate, carbolic 
acid, corrosive sublimate, and other metallic salts. It was not at all difficult to cause the organisms to lose color when cultivated with these chemicals; keeping them at a high temperature had the same effect. But in most cases, as soon as the organisms were returned to natural conditions the normal production of color was resumed; the "acquired character" was not inherited. Similarly transitory modifications of the color in other directions were produced. Such results were reached with infinite pains in a great number of experiments, with this organism and with other colored bacteria.

But in cultures in which the nutritive medium contained potassium bichromate, certain white colonies appeared which, when transferred to media without the chemical, continued to remain white, though there appeared also red spots amid the white. A long series of selections was carried on, choosing always the whitest parts of the colonies, but the tendency to return partly to the red condition could not be gotten rid of by selection. It was found that the longer the organisms were cultivated with potassium bichromate, the more firmly was the white established. When it first appeared the white color disappeared again as soon as the organisms were restored to normal surroundings; later the white became hereditary, though there was always a tendency for some part of the colonies to produce the red color. Some similar results were reached also with other chemicals.

In these cases therefore we have a most interesting transitional condition. The hereditary character of the race has been changed, for now the colonies are largely white under the same conditions in which they were formerly red. But they still show a tendency to return to the original character.

But with another substance, corrosive sublimate, a white 
color was produced that was permanent. When the bacteria were restored to their natural conditions they remained white, no matter how long the culture was continued. And with certain other chemicals the bacterial color became permanently a darker red; although restored to normal conditions and kept there for hundreds of generations, the acquired dark color persisted.

This work proves therefore that in bacteria by the action of the environment definite changes that are hereditary can be produced. From a single race, by subjecting parts of it to these diverse agents, a number of hereditarily diverse races are obtained.

In this case the alteration is evidently a change in the chemical processes of the organisms. The red color of this bacterium is not in the body of the creature, but is due to some substance produced by it, which colors the material on which the organisms live. In the experiments the effective substances changed the chemical processes so that the bacteria no longer produced this substance, or produced one of another color.

Similar in the fact that they deal with peculiarities that are visible to the eye are certain experiments of Toenniessen (1915). He investigated a certain strain of the bacillus which produces pneumonia. This organism produces a quantity of mucus, which forms a thick envelope in which the cell is imbedded; the volume of this envelope is perhaps several hundred times that of the cell itself. When the organisms are cultivated for a long time in dense colonies on agar, the products of their nutritive processes collect, until they decrease the organisms' power to produce the envelope of mucus. After a time some of the bacteria are found with only a thin envelope, others with none at all.

If these modified bacteria are transferred to normal con- 
ditions, in which the products of metabolism are not allowed to gather, they usually at once produce the normal amount of mucus; the change was not a hereditary one. But if the organisms are kept for a long time under the unfavorable conditions (four weeks or more), some produce no mucus at all; and if these are restored to normal conditions, they and their descendants continue to be without the mucous envelope. The change has become hereditary. But it is still not permanent, for by special means the organisms can be caused to begin anew to produce the normal amount of mucus. This is most completely brought about through allowing the organisms to live for a time in a living animal, by infecting a white mouse. After passage through the animal's body the bacteria have regained their normal powers of producing the mucous envelope.

By long continued cultivation with the products of metabolism, using special methods, Toenniessen produced other changes that were permanently hereditary. The organisms gradually produced less and less mucus, so that successive gradations could be distinguished. At least three of the grades were independently hereditary; one had a mucous envelope a little smaller than normal; a second had a very small envelope; the third had no envelope whatever. Long continued cultivation under normal conditions left each of these three grades unchanged; even passage through the body of animals did not restore the organisms to the normal condition. The alterations produced were permanently inherited.

In this case, as in the former, we observe the striking fact that what seems outwardly the same modification may appear sometimes without being hereditary; sometimes as hereditary for a number of generations; sometimes as permanently inherited. The difference appears to depend on the length 
of time that the modifying factors have acted; the longer they act, the more decidedly hereditary become the changes they produce.

Many of the hereditary changes that have been produced in bacteria manifest themselves only in altered physiological activities. Bacteria break up many sorts of organic compounds, obtaining by the recombination of their components the energy necessary for their own vital activities. Diverse species or races thus decompose different compounds. In a number of cases it has been found that if bacteria of a particular sort are cultivated in the presence of a compound which they do not decompose, but which is not too unlike some compound on which they can live, in the course of time some of the individuals acquire the power of decomposing and living upon this unaccustomed substance. This power then remains hereditary, so that the descendants have it also, - even though they may be cultivated under conditions in which it is not exercised.

For example, Massini (1907) found that a certain bacterium which belongs to the group of which the typhoid bacillus is a member, had not the power of decomposing lactose. But if they are grown on a culture medium that contains lactose, after a few days certain parts of the colonies begin to grow rapidly, forming small nodules; and tests show that these are now decomposing the lactose. If these are removed to other media and cultivated for many generations without lactose, their descendants still retain the power of decomposing this substance, as is shown by replacing them on a medium with lactose.

This fact has been confirmed by many observers, and similar changes have been observed in other cases. Bacteria have been caused to acquire the power of splitting up lactose, dulcite, rhamnose and various other carbohydrates, though 
when first cultivated on these substances they had not this power. In many cases the cultures so tested have been derived originally from a single individual, so that there is no question but that there has been an actual change in the hereditary capabilities of a single race.

In most cases the change thus brought about is permanent; the descendants never lose the capability that they have acquired. But in some cases it has been found that long cultivation under other conditions causes the descendants to lose the power which their ancestors had acquired. ${ }^{2}$

The slightness and delicacy of the hereditary changes so induced, and the fact that they increase by gradations, is shown in certain other experiments of Wolf (1909). Certain peculiar organisms known as Myxobacteria form dense swarms on decaying substances. A species known as Myxococcus rubescens thus forms circular red patches on culture media. If from a single patch of these, two distinct cultures are made, and the two swarms are later allowed to come in contact, they flow together and form a single swarm. But if the swarms come from diverse but related species they will not unite, but remain sharply separate. Even within the single species named above it was found that swarms from diverse sources refuse to unite, so that there are diversities of race showing in this behavior. A large number of races, diverse according to this test, were isolated from the single species.

The possibility naturally suggests itself that such differences can be produced within a single race. This was attempted by Quehl (1906), and later by Wolf (1909). A single swarm was divided into a number of parts, which were kept under different conditions, on diverse culture

'An excellent summary and review of all such cases up to 1912, with a helpful account of methods, and important details, is given in the paper of Dobell (1912). 
media. Later the parts were brought together again, under uniform conditions, to see whether they would still unite, or whether they had become sufficiently diverse to remain distinct.

It was found that the parts might be cultivated for a long time under diverse conditions, without becoming so changed as to refuse to unite. The first investigator who studied the matter did not succeed in getting a single race to divide into two that were diverse.

Wolf continued the experiments, keeping the parts separated a longer time, and using many diverse cultural conditions; in particular he added various sorts of chemicals to the different cultures. In this way after long periods differences were produced within a single race. An example will make clear the important facts. In one experiment a single original race was divided into eight parts, which were cultivated on diverse media. At intervals it is necessary to transfer each stock to a new lot of its medium, in order to keep the organisms healthy, and it is convenient to use the number of transfers made as a measure of the relative time required for changes to occur. After a few transfers, each of the eight divisions was tried with all the others, and it was found that all would unite readily. This still occurred after fifteen transfers. After twenty-five transfers, it was found that a few of the parts refused to unite with some of the others. After thirty transfers there were refusals in more than half of the combinations, and after thirty-five transfers each division refused to unite with any of the others. Each of the eight parts of the original race had now become diverse from each of the others; eight different stocks had been produced from one. Before the change was complete there were many transitional conditions, in which there was a reluctance to unite, without a 
complete refusal; conditions in which union was incomplete, and the like.

After the differences had been brought about, the colonies were all restored to the same culture medium and to the same other conditions, and cultivated thus for a long time. They still refused to unite when brought in contact. The change produced was hereditary and permanent. After fifteen transfers under uniform conditions,--representing hundreds of generations of the organisms, - the diversities still existed.

Even when the organisms were cultivated separately for a very long time, but without diverse chemicals in the culture media, they ultimately became diverse. In an experiment of this sort, it required fifty-six transfers, occupying a year and a half, to bring about hereditary diversities within a single stock.

When the diverse stocks thus produced were examined under the microscope, no differences could be detected. The change was evidently in the intimate chemical processes of the organisms, not showing in any visible way. The case is of particular interest because it shows that hereditary changes may arise in most delicate shadings which gradually become more and more marked.

Besides the work which we have just described, there has been much experimentation upon induced changes in hereditary characters of bacteria, with special relation to virulence, to immunity, and the like. The production of "attenuated" strains of bacteria, weaker in their injurious effects on other organisms, is a not uncommon practice. But most of this work has been done without the precautions necessary. for establishing the results, from a genetic standpoint, as actual cases of the inheritance of induced modifications. But the recent critical work of Wolf and others, described above, 
largely validates this large mass of material; it shows that hereditary changes of the kind which appear to occur in much bacteriological work, actually do take place when the matter is studied with all the required precautions. Summaries of much of this work, with references to the original papers, will be found in the publications of Dobell (1912), Jollos (1914) and Pringsheim (1910).

\section{Modifications of Inherited Characters in Higher Protozoa}

A relatively small amount of work has of late been done on the modification of inherited characters in the larger and more complex forms of Protozoa; some of the results here perhaps throw light on the nature of the processes occurring.

In the parasitic flagellates Trypanosoma, facts similar to some of those above described for bacteria have been demonstrated. A good review of the facts has been given by Dobell (1912). One case introduces a new element, which possibly throws light on certain general relations. The trypanosome possesses, besides a typical nucleus, a small body known as the kinetonucleus (see Figure 27, 2). This structure is placed close to the inner end of the motile flagellum, and may have some relation to the activity of the latter. In reproduction, the kinetonuclei of the two progeny are formed by division of the kinetonucleus of the parent. In Trypanosoma brucei cultivated in mice, it was found that when certain chemicals were injected into the mice, the kinetonucleus of the trypanosomes disappears (Figure 27, 1). The animals now multiply as usual, but remain without kinetonuclei; this continues indefinitely. Thus by the action of the chemicals a stock has been obtained which differs structurally from the original race; 
and this diversity is inherited in ordinary reproduction by fission. The same result has been produced wth several other species of Trypanosoma.

In Paramecium, as well as in some other infusoria, many attempts have been made to so modify the organisms that they will live under conditions which normally kill them.
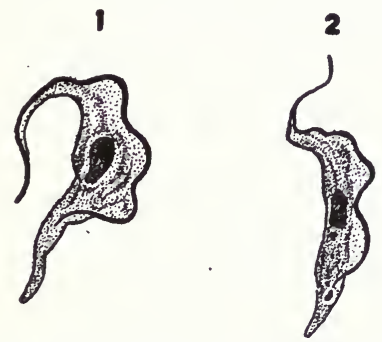

Figure 27. Trypanosoma brucei. 1. Individual from which the kinetonucleus has been removed, by treatment with chemicals. 2 . Normal form (the kinetonucleus is the dark body near the lower end). After Werbitzki, from Dobell, 1912.

All such attempts, if they are successful, involve a change in the animals and the inheritance of this change by the progeny. For since they reproduce every twenty-four hours or oftener, the acclimatization would not last longer than that period, if the progeny returned at once to the original condition.

Most experiments in acclimatization attempt to accustom the organisms to high temperatures; or to poisons of various kinds. One sometimes gets, from reading, the impression that it is easy to do this. But most persons who try it are greatly disappointed. The organisms appear quite unchanging; if the experiments are not carried on for a very long time, and the change of conditions made with extreme 
slowness and gradualness, the animals usually show no acclimatization; they die as soon as the temperature or the poison reaches the intensity which was destructive to them at the beginning. But with extreme patience and perseverance, a change gradually appears. Perhaps the most thorough experiment of this sort ever made was carried out long ago by Dallinger (1887); he continued the process of acclimatizing the animals to higher temperature for seven years, and reached more striking results than anyone else has attained.
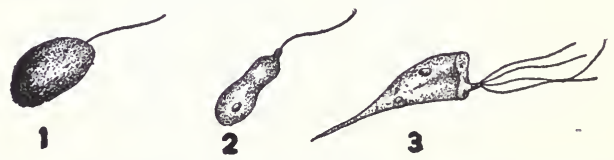

Figure 28. Organisms used in Dallinger's experiment on the effects of high temperatures. 1, Monas Dallingeri; 2, Dallingeria Drysdali; 3, Tetramitus rostratus. After Dallinger.

Dallinger worked with three minute flagellates that live in putrefying infusions: Tetramitus rostratus, Monas Dallingeri, and Dallingeria Drysdali (see Figure 28). The temperature at which they flourished was $60^{\circ} \mathrm{F}$. $\left(16^{\circ} \mathrm{C}\right.$.); they were killed at once by a temperature of $142^{\circ} \mathrm{F} .\left(61^{\circ}\right.$ C.). But at least one of them, Dallingeria, formed spores which could resist (in fluids) a temperature of $220^{\circ} \mathrm{F}$. (104 ${ }^{\circ}$ C.).

Dallinger undertook to accustom the animals to higher temperatures. He found that up to $70^{\circ} \mathrm{F}$. little difference was observable in the life and growth, although the animals lived better under the later increases if the change from $60^{\circ}$ to $70^{\circ}$ was made very slowly. Above $70^{\circ}$ it became necessary to proceed with extreme slowness; Dallinger raised the 
temperature by only two degrees each month. At $73^{\circ}$ many died, but as the temperature remained at this point for two months, the remainder recovered their vigor. At $78^{\circ}$ a critical point was reached; as many of the animals died, the temperature was lowered to $77^{\circ}$ till there was recovery, and by repeatedly alternating the temperature between these two points, in eight months the animals lived healthily at $78^{\circ}$. They now underwent a most interesting visible change; the protoplasm became filled with small vacuoles. These continued for a month or two, then the vacuoles disappeared. Now the temperature could be farther increased; in three months it was raised to $80^{\circ} \mathrm{F}$.

By a continuation of this slow and painful process the animals were finally brought to live vigorously at a temperature of $158^{\circ} \mathrm{F} .\left(70^{\circ} \mathrm{C}\right.$.). There were repeated critical points, at which the animals had to be kept for months before further advance could be made. In several of these there was a renewed formation of vacuoles in the protoplasm, the vacuoles finally disappearing. After these periods the raising of the temperature could continue more rapidly. To bring the animals to 158 degrees, seven years were required. The experiment was then most unfortunately ended by an accident.

No such long continued experiment has ever been carried through since this work of Dallinger. It is clear that not only had the organisms of a given generation been changed, but they transmitted the change to their offspring. For at the end of any period of 24 hours a totally new generation was present. At the beginning of the experiment all the individuals were destroyed by a temperature of $78^{\circ}$; while at the end they lived and flourished at a temperature above $150^{\circ}$.

Yet it is to be remembered that even at the beginning 
the animals could form spores which resisted a much higher temperature $\left(242^{\circ}\right)$ than that to which the active animals were finally accustomed. In-transforming from active animals to spores, the protoplasm must go through some process which makes it more resistant to heat. It seems probable that during the acclimatization the protoplasm of the active animals went through a similar process. It has been suggested that the essential point in both cases is the getting rid of a certain proportion of the water in the protoplasm, leaving it denser, for protoplasm containing little water is as a rule less injured by heat than when it contains much water. This new physical condition of the protoplasm must then have persisted through reproduction, and so been handed on to the off spring.

Some effects of temperature in altering a different manifestation of the hereditary constitution have recently been studied by Middleton (1918). Progeny of a given individual of the infusorian Stylonychia pustulata were divided into two sets; one set was kept at a high temperature, the other at a low temperature. Those at the high temperature (about $30^{\circ} \mathrm{C}$.) divided more rapidly than those at the low temperature (about $10^{\circ} \mathrm{C}$.). After various intervals, members of the two sets were brought to a common intermediate temperature, and their rates of fission compared.

It was found that the stay in diverse temperatures had altered the hereditary constitution so as to give diverse rates of fission in the two stocks. After about thirty days in the different temperatures, the set that had been kept at high temperatures continued to divide more rapidly than the others, even though both were now at the same temperature. But after longer periods in the diverse temperatures, -after two or three months or more,- there was a change 
in the inherited effects. Now when both sets were placed at intermediate temperatures, the set that had been kept at the higher temperature divided less rapidly than the set that had been kept at low temperature. This difference persisted for as long a period as the stocks were retained,about two months.

Other evidence showed that the high temperature gradually injured the stock, so that in the course of time the high temperature set came to divide less rapidly even while subjected to high temperature. At the end of six months those kept at the high temperature all died out, while the other set was still vigorous. The persistent low fission rate of the high temperature set when restored to normal temperature was apparently a manifestation of this injury. The latter, whatever its nature, was inherited in the vegetative reproduction.

Dallinger did not determine how long the acquired resistance to heat would have lasted if his animals had been restored gradually to lower temperatures; and no study for long periods of the permanence of the effects observed by him was made by Middleton. But this matter has been studied particularly in Paramecium, by Jollos (1913 a, 1914). He attempted to accustom Paramecium caudatum to higher temperatures, and to increased concentration of certain compounds of arsenic. Some races resisted acclimatization completely. In others after long periods the animals could stand somewhat higher temperatures or higher concentrations than before. But when they were returned to the normal conditions, they lost their acquired immunity almost at once.

In other cases the animals acquired a resistance to poisons which was retained by their descendants for many generations. Thus, in a certain race $B$ the animals were killed 
when 1.1 parts of a standard solution of arsenic was added to $\mathbf{1 0 0}$ parts of the water. By a gradual process they were rendered resistant to 5 parts of this same solution to 100 of water. When they were again cultivated in fluid without arsenic, they retained their resistance unchanged for seven months, or at least 200 generations. But in the eighth month it was found that the resistance was partly lost; they could now stand only 4 parts of the arsenical solution in 100. The resistance continued to decrease gradually, until at the end of ten and a half months they had entirely lost the resistance to arsenic that they had acquired, so that they were killed by the same weak doses that had been destructive at the beginning. In many other cases Jollos thus produced modifications of the power of resisting chemicals, which thus lasted for months, but finally disappeared. He found that if the animals were kept under constant conditions their resistance lasted much longer than was the case if they were subjected to many changes of temperature and food.

One particular phenomenon did away immediately with the acquired resistance; this was conjugation. Jollos found that after the animals had acquired resistance to a considerable concentration of arsenic, this resistance was completely lost as soon as they conjugated. To this fact Jollos attributes a deep significance. He believes that it shows that the modifications thus produced and for a long time passed from parent to offspring by fission are in reality very different things from the permanently inherited characteristics of the species. These characters - the typical form, structure, and physiology-are inherited not only in fission, but also in the changes which follow upon conjugation. A change in these characters, - a permanent change in the inheritance,-Jollos would call a mutation, while these 
changes that are handed on only through vegetative reproduction he calls modifications; the two he believes to be of essentially diverse nature. Such a "mutation" Jollos believes that he saw in a single instance in Paramecium. In one of his cultures kept at high temperatures there appeared individuals which were much more resistant to heat than most of the animals; they could be cultivated at $39^{\circ}$ C., which soon killed the others. These individuals retained their high resistance even after conjugation; it had become a permanently inherited character. In no other case was a modification retained through conjugation. Jollos holds that practically all the changes in bacteria and other Protista, which we have described above, are merely instances of these temporary modifications.

That there is such a difference in principle between the two things,--between modifications that are passed on only in fission, but disappear as soon as there is sexual reproduction, so that they cannot be said to form part of the really hereditary characters of the stock,-between these and the really hereditary characters,- - cannot yet be considered established. If there is such a difference, one can hardly refrain from bringing it in some way into relation with the two nuclei. Since in fission the new active nuclei are produced by division of the active nucleus of the parent, one might naturally assume that the seat of the temporary modifications is in the active or macronucleus, while the reserve nucleus (micronucleus) has not been affected. Thus would be accounted for the fact that at conjugation, when the macronucleus disappears and is replaced by the micronucleus, the modifications also disappear; they go with the macronucleus. But we now know from the work of Woodruff and Erdmann that the macronucleus disappears and is replaced from the micronucleus at intervals even 
without conjugation; the modifications should therefore disappear at such times. The fact that they do not indicates that the distinction is not one depending on whether the seat of the modifications is in the macronucleus or the micronucleus; it leaves the distinction indeed with no very intelligible foundation. It appears possible that modifications which are now known to last for months might endure still longer, and become as permanent as any character, if the conditions producing them lasted for much longer periods. The fact that the modifications sometimes disappear at conjugation may be due to the fact that variations of many sorts occur as a result of conjugation, as will be set forth in our account of that matter. The number of cases in which these phenomena have been studied is very small; too small for basing positive conclusions on these points.

All together, the studies of the effects of external agents on heredity in the Protozoa show that changes in the hereditary characters are in this way produced only most slowly and rarely. The organisms are found most resistant to such changes; any alteration produced in a given generation is usually compensated for in the next generation. Almost every investigator of the matter passes through a long stage in which he can hardly resist the conviction that no hereditary changes can be brought about in this manner. But if work is continued for very long periods of time, the hereditary constitution of the stock is seen to gradually yield; at first only in a slight degree and with results that are transitory. The differences finally become so fixed that they are transmitted in the ordinary reproduction by fission. After conjugation, with its extreme physiological alterations, and production of new combinations of inherited characters, the inherited environmental effects are frequently no 
longer clearly in evidence; possibly they are masked by the new combinations occurring; possibly actually lost.

In general the results of the work suggest that the many slightly differing stocks found in any one of these lower organisms may owe their origin partly to the inherited effects of long continued environmental diversities. 


\section{V}

The Natural History of Mating. Sex, Its Nature and Consequences. Sex in the Protozoa: Is Sex Coextensive with Life and Necessary to Its Continuance?

WE have dealt with heredity and other genetic problems in the cases where there is but a single parent; we now turn to reproduction where there are two parents instead of one. The mating of two individuals that occurs at times in almost all organisms is one of the most extraordinary processes in nature; it has the effect of complicating tremendously all biological questions. Volumes have been written as to its purpose and meaning.

Some tell us that it is unscientific to ask as to the "purpose" or "object" of any process; Dobell (1914) has made this point with relation to all such discussions of mating. The criticism is justified, so far as the method of expression goes, and the literal implications of that method of expression; science cannot deal with purposes or ends, save in the case of conscious human purposes. Nevertheless, a really scientific question is often hidden under this form of expression. What it really means is: What difference does it make whether this process occurs or not? Any question as to "purpose" or "object" that can be put in this form is a scientific question in spite of its teleological clothing; any teleological question that cannot be put in this form is no affair of science. To ask what difference this phenomenon makes, leads at once to experiment; a question that could 
not be settled by any conceivable experiment is not part of science.

So the question in which men have been interested in relation to mating and fertilization is: What difference does it make whether this occurs or not? This is strictly a question of observation and experiment, on the same footing as the question: What difference does it make whether animals take food or not?

When we ask this question regarding the union of two individuals or parts of individuals which we call mating and fertilization, we find that there is hardly another phenomenon in biology that so alters the whole face of things. Biology would be a relatively simple subject if there were no periodic unions of diverse individuals, with the accompanying processes. This union has results of so many different kinds, some immediate and obvious, others remote and hidden, that we find little agreement in the accounts of its fundamental features given by different investigators.

A picture of what happens in the higher organisms that we are familiar with will bring the question sharply before us. Mating here involves two diverse individuals that we call male and female, and two diverse germ cells, which we likewise call male and female. But this is not the end; the final mating is between certain parts of the cell, after the two germ cells have joined to form one. The cell now contains a set of pairs of small visible packets of chemicals, the chromosomes (Figure 29). These mate in pairs (Figure 29, D, E, F) and again separate. This is the final and elementary action of mating; the union of these chromosomes contains the secret of sex and of mating. The union of the two diverse germ cells forms the starting point for the development of the new individual.

Several questions of general interest come into view in 

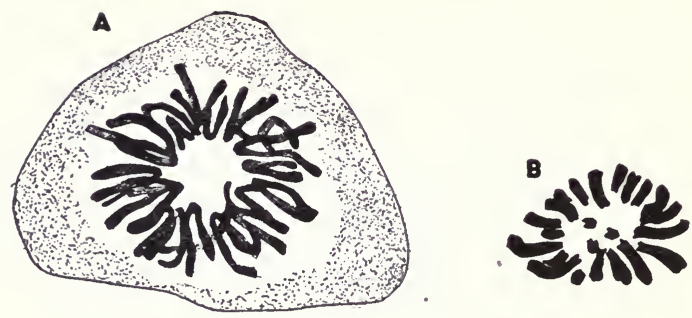

\section{)$(1 / 111) 11 \ldots$ \\ c

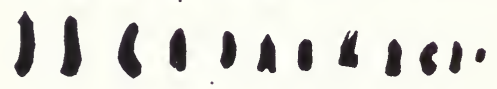

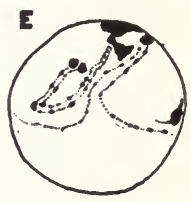

\section{$.000 \cdot \cos 160.0$}

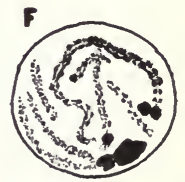

Figure 29. Chromosomes and their mating. A. Nucleus containing the chromosomes, from the salamander. B. The 23 chromosomes in a single cell of a male grasshopper, as seen under the microscope. C. The chromosomes of B drawn separately and arranged so as to show that the group consists of a series of 12 , the two members of each pair being of the same size and form. One chromosome (fifth from the left in the upper row) is without a mate in the male; in the cell of a female it has a mate. D. The members of the pairs after mating. Each of the 12 structures (save one) is formed by the union of the two members of a pair. $\mathrm{E}$ and F. Details of the mating of the chromosomes in the cells of another species of grasshopper. In E only two chromosome pairs are seen; in the pair to the right mating side by side has begun, but is not complete. In F several pairs are shown, fully mated. Each of the granules of which the chromosome is composed mates with a granule of corresponding size and position in the other chromosome. B to D, after Robertson, 1908. E and F, after Wenrich, 1916. 
regard to this strange process. First, is this union a necessity of life? That is, could and would life continue without its occurrence? Could and would development occur without it? Is there some single general result produced by mating,- - something as general as the production of energy through the taking of food? If there is, what is this single general effect of mating? This is the real point underlying the much-discussed question: What is the purpose of mating?

Certain other questions arise from the differences between the two sexes. What is the nature of this difference? That is, is there a single kind of chemical or physiological difference between the sexes, wherever sex differences occur; a fundamental diversity of which all other sex diversities are consequences? And does this diversity exist whenever there is union of individuals or cells or parts of cells? Are the two chromosomes that unite diverse in this manner? And are all the unions that occur a consequence of such diversity? Or may two precisely similar individuals or cells or chromosomes unite at mating? Again, is this sex difference coextensive with life, so that all living things are composed of two classes of substance, male and female, as some have asserted? Or is sex difference something that pertains only to certain kinds of organisms; perhaps something that has arisen during evolution, like the difference between two species of plants or animals?

We shall examine the facts in the Protozoa in their bearing on these questions, and shall try to determine with which answers these facts agree best. We will take up the process of union first in what is perhaps the best known and most instructive case in these lower organisms, in the infusorian Paramecium. Then we shall compare what happens in this animal with what occurs in others, keeping in mind throughout the fundamental questions that we have set forth. 
As you recall, Paramecium multiplies for many generations in single lines, the offspring having but one parent. Then mating occurs; the animals place themselves with their oral sides together and become partly united (Figure 6). The surfaces of the two adhere, and at a certain spot the interior protoplasm of the two comes into actual union. The main things that then occur are those shown in Figure 8 (page 26), and in Figure 30. The old active nucleus (macronucleus) breaks in pieces and is gradually absorbed, like so much food; it disappears completely. This really requires a long time, so that fragments of it are still found in later stages, but as this has no importance, these fragments are omitted from the figures in order not to confuse them. The single small reserve nucleus (micronucleus) divides twice, into four (Figure $30, \mathrm{~A}, \mathrm{~B}, \mathrm{C}$ ), and three of these are absorbed and disappear (C) like the macronucleus. Then the remaining (fourth) one divides into two parts (see Figure 40). Of these two parts, one lies a little nearer the surface of union of the two conjugants, and is a little smaller than the other. This one begins to move toward the opposite conjugant; it is therefore commonly spoken of as the "migratory" half nucleus. In the other conjugant the same thing happens, so that the two migratory half nuclei meet and pass each other at the boundary between the two conjugating individuals (Figure 40, B, C, D). Each of the two stationary halves remains in place till the migratory half nucleus from the opposite individual reaches it; then the migratory and stationary half nuclei unite (Figure 30, E; Figure 40, E, F).

Thus the general upshot of the process is that the two mating animals exchange halves of their micronuclei, and at the end each has a micronucleus composed of substance partly from one mate, partly from the other. This is evidently the central point in the conjugation. 


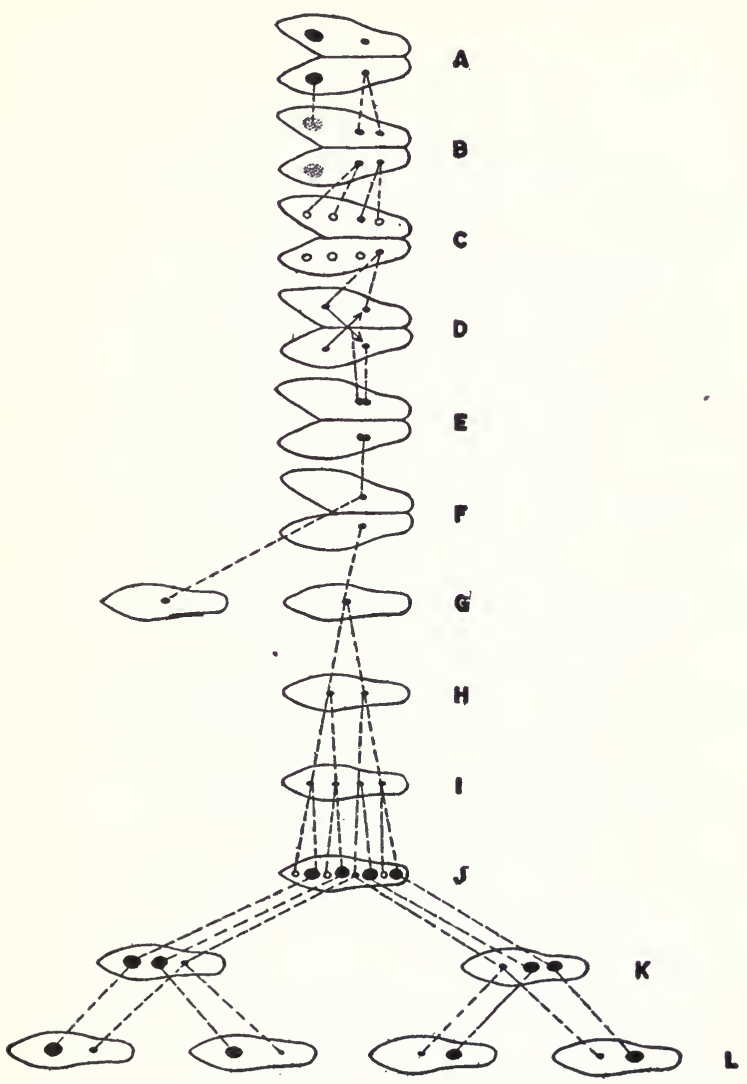

Figure 30. Diagram showing the chief processes in the conjugation of Paramecium caudatum. The larger black bodies are the macronuclei; the smaller ones the micronuclei. The clear circles are the micronuclei that disappear. The connecting lines show the origin by division of the various structures. After the separation of the two members of the pair, at $G$, only one of them is followed farther; the other goes through the same processes ( $\mathrm{H}$ to $\mathrm{L}$ ). 
After the union of the two half nuclei the two mates separate; we may now call each an ex-conjugant. Now a set of peculiar pirocesses occurs, with the result of restoring, through two divisions of each ex-conjugant, individuals having the same structure as did the mates before conjugation. Some of the details of the process may turn out of great significance, though at present their meaning is not clear.

At separation each ex-conjugant has a single nucleus formed by the union of the two half nuclei (Figure 30, F). This single nucleus divides, producing two; these divide, producing four; these again divide, producing eight. These eight are all present in the single ex-conjugant (Figure $30, J)$. Now of these eight three dissolve and disappear, leaving five in the single individual. One of these, as it later turns out, is the nucleus from which all the micronuclei of later generations arise by division; the other four later form four macronuclei of four individuals of later generations,-each increasing greatly in size.

Now the single ex-conjugant divides, producing two individuals. At the same time the single micronucleus divides into two halves, each passing to one of the off spring. Each of the two offspring also receives two of the four macronuclei, which are now enlarging (Figure 30, K). Next each of these two offspring divides anew with repetition of the division of the micronucleus, while of the four progeny each receives one of the four macronuclei. Thus after two divisions of the ex-conjugant, off spring are produced with a single macronucleus and a single micronucleus (Figure 30, L), -like the individuals before conjugation. Each of the four individuals arising from an ex-conjugant has one of the four macronuclei that were present in the ex-conjugant. But the micronucleus of each of the four is derived by division from a single micronucleus present in the ex-conjugant. 
What significance is to be attached to the dissolution of three of the eight nuclei originally present in the ex-conjugant is not clear; nor is it clear what is meant by the diverse method of origin and distribution of the new macronuclei as compared with the new micronuclei; to this point we return later.

The many details in these processes that occur after separation of the ex-conjugant must not be allowed to obscure the essential features. These are simply that the new micronucleus formed by union of the half nuclei divides so as to produce new active macronuclei and new reserve micronuclei (Figure 30, G to $\mathrm{L}$ ); and the animals continue to divide by fission, as they did before conjugation,-each of the off spring getting a single active nucleus and a single reserve nucleus.

In this process of conjugation are involved all the problems of sex and of mating. It is of interest to examine it in connection with the general questions which we proposed in our introduction to this lecture, and with some of the commoner answers to these questions. To this we turn.

What are the results of this mating? What difference does it make to the organisms or to the race which they make up?

As we saw in our first lecture, the best known theory as to the effect of such conjugation is that it rejuvenates the organism; that it gets rid of the effects of age. But in what way can it have this effect? There are two possible answers to this question. One is that the rejuvenescence is a result of the replacement of the old active nucleus by the reserve nucleus. But for this no union of two individuals is necessary, either logically or in fact; we know now that such replacement occurs without union. This answer therefore gives no explanation of the fact of union; what we are 
interested in now is precisely the question as to the effect of the mating as distinguished from the process of replacement. A second answer to the question as to how rejuvenescence is brought about holds that it is due primarily to this union of two individuals or of two nuclei. In the minds of many that hold it the grounds for this belief are undefined; Maupas (1889, p. 486) in his great work on rejuvenescence, in which he maintains this theory, avows distinctly that he cannot see how the union of nuclei should produce rejurenescence, though he believes that it does. But there has grown up a theory as to how the rejuvenescence is brought about by union; a theory that is rather generally held. This theory depends upon the farther theory that there is a fundamental difference between the male and female sexes, and that this essential sex difference is always present in mating, and is the basis of rejuvenescence. We know indeed that in many organisms the differences between the sexes are not mere superficial diversities, but that the two sexes differ in every cell of their bodies, and the observable difference lies precisely in the nuclei, which we know to be fundamentally important parts of the organism. In the commoner cases, including man, the nuclei of the female have one more chromosome than have those of the male (Figure 31, A and B); in other cases one chromosome of the female differs from the corresponding one in the male (Figure 31, C and D). Such a visible structural difference necessarily means further a diversity in the most fundamental and intimate physiological processes of the two sexes; in the chemical changes that determine the nature of life. These chemical changes we observe to occur in the physiological interaction between the nucleus and the rest of the protoplasm.

What are the fundamental physiological differences be- 
tween the sexes? In a general way the male in most organisms is the more active and the less inclined to store up reserve food in its tissues; the female less active and given to storing up more reserve nutrition. These differences are of course seen most clearly in the germ cells of the two sexes; the typical male germ cells are minute and actively motile, while the female germ cells (eggs) are large and inert, with much food material stored in them. Less marked
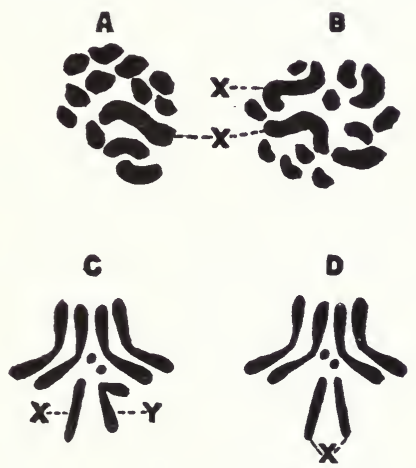

Figure 31. Differences between the chromosomes of the nuclei in the two sexes. A and B, male and female chromosome groups, respectively, of the hemipterous insect Protenor, after Wilson (1910). The nucleus of the female (B) has two of the large chromosomes $x$, while the male (A) has but one.

$\mathrm{C}$ and $\mathrm{D}$, male and female chromosome groups respectively, in the nuclei of the fruit fly Drosophila, after Morgan (1916). The male group (C) has one bent chromosome (Y) in place of one of the straight ones (X) of the female (D).

differences of the same general character distinguish the male and female individuals that produce these germ cells.

Many attempts have been made to express such differences in general terms. Geddes and Thompson (1880) say that in the female the preponderating process is that of anabolism, 
or the building up of organic material, with the storing up of energy, while in the male the prevailing process is catabolism, or the breaking down process by which energy is set free,- - this energy showing itself in greater movement. Or the male has been called "kinetic," the female "trophic"; or the male progressive, the female conservative; or it is said that in the male the animal functions prevail, in the female the vegetative functions.

In recent times emphasis has been laid on a parallel diversity observable between certain parts of the cell or parts of nuclei. When the cell divides, certain parts seem to actively initiate and carry on the movements that bring about division, while other parts are passively moved by these active portions. In many organisms a structure called the aster, which in some cases itself appears to be derived from a small body called the centrosome, sets up at the time of division a great activity in the protoplasm, forming the spindle, and taking the lead in the activities of cell division (see Figure 32). Another portion of the cell, comprising the part commonly called the nucleus, and particularly the chromosomes, is apparently passively moved by these active portions. In some Protozoa there are two nuclei, one of which, the so-called kinetonucleus or blepharoplast (see Figure 27 ), is connected with the organs of motion, and is supposed by some to correspond to the centrosome. The other, the so-called trophonucleus, appears more passive, and is held to have functions connected primarily with metabolism. The materials of which these two parts are composed are called respectively kinetoplasm and trophoplasm, and it is held by the upholders of this doctrine that these two kinds of material are present, either mingled or separate, in every cell that can divide. ${ }^{1}$

${ }^{1}$ A résumé of the facts on which this notion is based is found in the paper of Dobell (1909). 

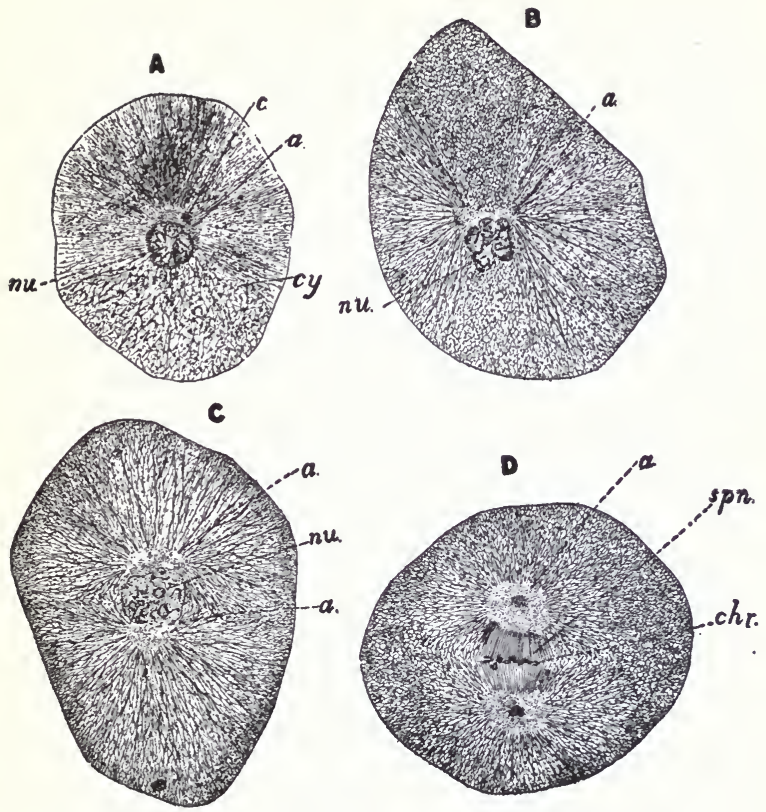

Figure 32. The active and passive portions of cell and nucleus in the egg of the whitefish. A. Egg just before division; with centrosome (c), aster (a), nucleus (nu.) and cytoplasm (cy.). A set of radiations have been formed about the aster as a center, indicating great activity. B. Later stage; the aster has begun to divide into two. C. Still later; the aster has separated into two with the nucleus between them. The activities of the asters have begun to invade the nucleus. D. The nucleus has been transformed by the activities of the asters into a spindle-like body, with the small chromosomes (chr.) ranged across its middle.

These two kinds of material, with their contrasted ways of behaving, are held by many to form the basis for maleness and femaleness, so that sex diversity is coextensive with life, 
although both sexes may be present in a single nucleus. But individuals or cells in which the active material or kinetoplasm prevails are male, while those in which the nutritive material or trophoplasm is preponderant are female.

According to such theories, for vigorous and successful life, an organism must have both these substances or properties,-both maleness and femaleness,-in fitting proportions. And it is the gradual dislocation of the required proportions that gives rise to the necessity of periodical unions of diverse cells or nuclei, and to the rejuvenescence that results from such unions. It is commonly held that as life goes on, certain individuals or cells or parts of cells develop, perhaps by accidental inequalities in division, farther and farther in the direction of maleness, of activity, of energy-production; that is, the kinetoplasm exceeds greatly the trophoplasm. Others develop farther and farther in the female or vegetative direction; toward a decrease in activity; toward a storing up of food and energy; toward a great preponderance of trophoplasm.

After this divergent development has gone on for a time, in each case, according to such theories, a stage of too great specialization is reached; a stage of lack of balance; the male has developed too far in one direction, the female in the other. Life can therefore no longer continue vigorously and normally; the chemical changes go awry; the process that we call aging comes on; reproduction ceases; and life must end unless compensatory changes occur. Such compensatory changes are brought about by a reunion of the separated male and female substances or tendencies; the balance is thereupon restored. In other words, rejuvenescence is produced by mating; reproduction can now go on as before; the cycle of youthful life begins anew.

According to this way of looking at the matter, there is 
an actual attraction of some sort between the two kinds of substance when separated, and this it is which causes them to unite. In the case of entire individuals, and particularly in higher organisms, this attraction must of course work through many indirect means, but in the actual union of male and female nuclei or of chromosomes (Figure 29) it must show itself in some direct and simple manner, as when two chemicals unite (compare Doflein, 1911, page 260). The cause of the union is the diversity. Thus, according to this view, the attraction of the sexes has its basis in the foundations of life.

To be consistent, this theory has to maintain that in the union of the chromosomes in pairs, which appears to be the elementary act in mating, there is this same diversity between the two uniting members, and that this is the cause of their union.

Further, it would appear that according to this view, growing old is fundamentally a different thing in the two sexes; in the female it would be the consequence of an excess in the vegetative functions; in the male a consequence of excess in the kinetic functions. This consequence $I$ have not seen drawn, but it appears an unavoidable one if the theory is held. Doubtless in each sex it could be held that some other processes essential to life are interfered with through these changes, in such a way as to give similar outward signs of age, such as we see in the two sexes of higher organisms.

Let us now turn back to the conjugation of Paramecium and see its relation to such ideas.

First, of the two individuals that mate, each plays the part of both male and female. Each furnishes a smaller, active half nucleus, which moves over to join the larger inactive half nucleus of the other individual; this active half nucleus evidently takes the rôle of the male germ cell. But 
each individual of the pair likewise produces a larger inactive half nucleus, which remains passive and is sought out by the smaller one; this inactive nucleus plays the rôle of the egg in ordinary fertilization. If there is a fundamental sex difference between the two uniting half nuclei, we must call the active one male, the passive one female. But the two individuals that unite both produce male and female nuclei; each would have to be characterized either as both male and female, or as neutral. There appears a difficulty here, for why, if the two individuals are alike, should they be drawn together and mate? According to the theory it is unlikeness of sex that brings about mating.

Attempts have been made to show that the two individuals which conjugate really are of preponderatingly different sexes; that one exceeds the other in maleness, the other in femaleness, if we may so speak; this would avoid the difficulty we have mentioned. Calkins (1902) pointed out that often only one of the two individuals that have mated reproduces freely, the other reproducing but weakly or dying without reproduction; Miss Cull (1907) showed that this happens in a great number of cases. The individual that produces young after mating would be mainly female, while the other would play the rôle of male. Of course the differentiation of sexes was not held to have gone far in such a case; all that is needed is that one should be, as it were, more female than the other; then the diversity would operate to produce mating and consequent rejuvenescence.

This idea is rendered plausible by the fact that in some other infusoria, such as Vorticella and its relatives, there is an observable difference between the two individuals that conjugate. One of the two mates is small and active, taking the usual rôle of the male, while the other is larger and remains quiet, taking the part of the female (Figures 33 


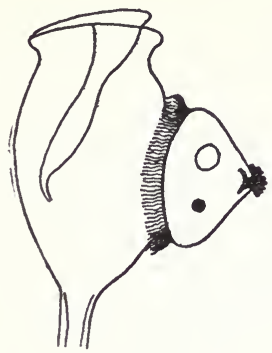

Figure 33. Beginning of conjugation in Epistylis, after Wallengren, 1899. The small active male is attaching itself to the side of the large inactive female.
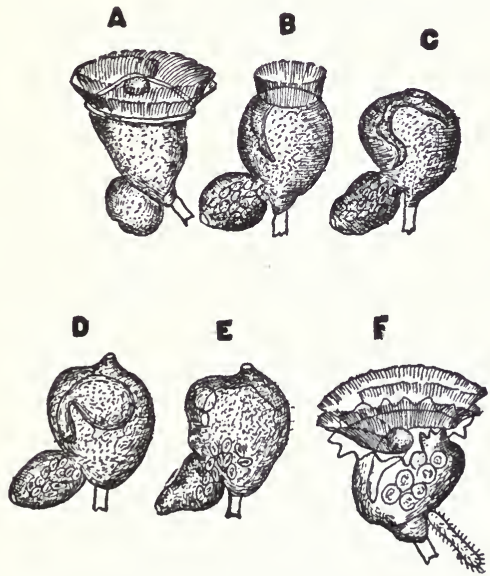

Figure 34. Successive steps in the process of conjugation in Vorticella nebulifera, after Maupas, 1889 . The small male unites with the base of the female; its protoplasm entering the body of the latter, so that finally only the outer layer remains outside (at F), finally dropping off. 
and 34). In these cases too the "male" completely unites with the female, the two merging into one (Figure 34), just as happens with the two germ cells of higher organisms; they do not separate and continue their individual lives, as the two mates do in Paramecium. Each of the mates in
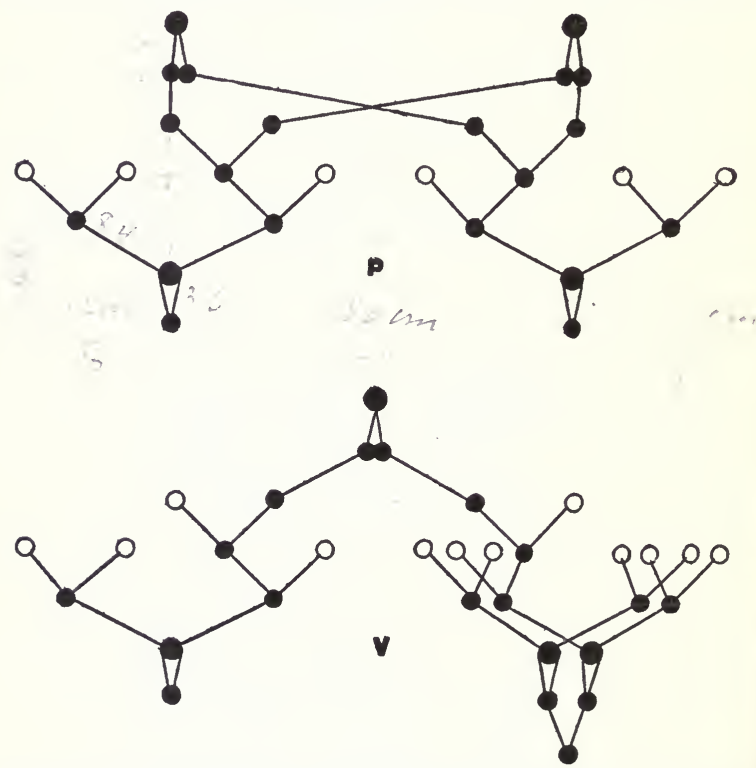

Figure 35. Diagrams of the micronuclear processes in conjugation, in Paramecium (P) and Vorticella (V), up to the formation of the new micronucleus by the union of the migratory and stationary half nuclei. In each case the original micronuclei of the two mates are shown below, and successive stages and divisions are shown in passing upward. The micronuclei that dissolve and disappear are shown as clear circles. In Vorticella (V) the divisions in the small free individual (microgamete) are shown at the right; those of the large stalked individual (macrogamete) at the left. Based on diagrams by Maupas, 1889. 
Vorticella produces just before the fertilizing process two half nuclei, as Paramecium does, but in the large "female" one is absorbed and disappears, while in the small "male" likewise one is absorbed and disappears. The remaining half nucleus of the "male" then passes into the "female," and unites with its remaining half nucleus. (See the diagram of the process in comparison with that in Paramecium, in Figure 35).

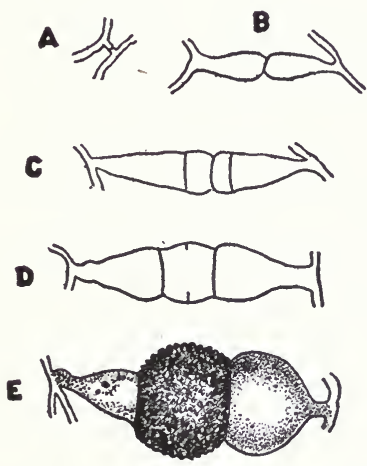

Figure 36. Mating in the mould, Mucor, after De Bary. A to E, successive stages. The large black body at $\mathrm{E}$ formed by the union of the ends of the two branches, is the zygospore, which later separates off, and produces a new plant.

In Paramecium, it was held by Cull (1907) that there is a less advanced stage of a similar process; though the two individuals look and act alike, they still are different, since after mating one reproduces more vigorously than the other.

Cases are known in which the individuals of a species are really physiologically diverse as to sex, although in appearance they are alike. This has been worked out fully by Blakeslee (1904) in the common moulds, Mucor. Here the 
filaments produce small side branches, which are separated off by a partition as club-shaped germ cells or reproductive bodies (see Figure 36). These reproductive bodies unite with corresponding bodies from another individual plant. By test it is found that these reproductive bodies are not indifferent as to their mates; with reproductive bodies from certain other plants they will not unite. Thorough study shows that there are two classes; that those of one class will not unite with those of the same class, but will mate with those of the other class. The characteristic behavior of the two sexes occurs, although the two show no external differences (save that in some cases the "female" plants grow somewhat more luxuriantly than the "male" ones).

Such cases show that the physiological distinction of two sexes may exist without any structural difference. Is this so in Paramecium? The case here turns out not to be so probable a one for sex difference as it at first seemed. It is true that sometimes after mating one individual reproduces freely, while the other does not. But on the other hand, sometimes both individuals reproduce freely; sometimes both reproduce feebly; sometimes neither reproduces at all. That is, there are great differences among the individuals that have conjugated, in respect to these (and other) matters. The question therefore reduces itself to this: Are the two that have mated any more unlike in these respects than are any two ordinary individuals that have not mated together? For example, if one multiplies strongly, is its mate more or less likely than the average individual to multiply weakly or not at all?

This led Lashley and myself to investigate the matter (Jennings and Lashley, 1913). If we have a large number of cases it becomes a mathematical problem to determine whether the two members of a pair are more unlike than 
usual; there are definite methods for solving such a problem. Using a large number of experiments, we found, rather unexpectedly, that the two individuals that have mated are more alike in all these respects, not less alike. If one member of a pair dies, its mate is more likely to die than are the mates of individuals that live. If one member multiplies feebly, its mate is likely to multiply feebly, too. If one member multiplies vigorously, its mate is likely to multiply vigorously also. All these relations are quite the opposite of what would be expected if there are pronounced sex differences between the members of pairs. But from an entirely different point of view, they are what might well be expected. The two mates have exchanged parts, so that after conjugation each is, as it were, half composed of material from the other (this is strictly true of the nuclei), so that it is natural that they should become alike. This brings into vie $N$ another result of mating; one of the first importance. Mating causes the progeny of the two individuals that mate to be alike. This point we shall take up later; here we shall look farther into the question of sex differences.

We have therefore no positive evidence of a difference of sex between the two members of a pair of Paramecia. On the other hand, perhaps it can hardly be maintained that such differences are disproved. If all we hold is that some difference in degree of "maleness" or "femaleness" is what produces mating, it becomes extremely difficult to test whether this holds or not. In this connection there are a number of facts, some of them very curious, that require consideration.

In many lower organisms, germ cells are formed by the division of a single cell, and so far as anyone can see, these are all alike. Further, so far as anyone can determine, any 
two of these can mate,-just as apparently any two individuals can mate in Paramecium. This is particularly the case with many lower plants (Figure $3 \%$ ). It is true even in many of the moulds, in some species of which, as we have before seen, physiological sex differences exist, even though there is no visible difference between the sexes. In other species of moulds, no such physiological sex difference exists; a given individual can mate with any other individual of the species.

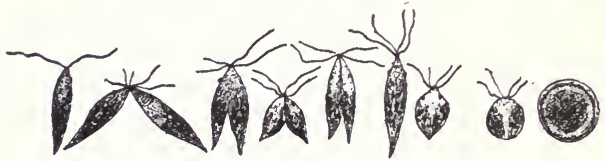

Figure 37. Mating of similar cells, from the alga Stephanosphaera; successive stages from left to right. The two unite to form a single reproductive body or zygote (at the right). After Hieronymus, from Doflein, 1911.

From such cases, common in lower organisms, it is natural to draw the conclusion, which many have drawn, that in these lowest creatures there is no difference of sex; that sex has arisen as evolution progressed; that therefore sex is not coextensive with and fundamental to life.

On the other hand, some have held that the very fact that two unite is sufficient proof that they are sexually diverse; this for example is the view taken by Minchin (1912) in his Introduction to the Study of the Protozoa and by Coulter in his interesting work on the Development of Sex in Plants (1914). ${ }^{2}$ If this argument is advanced as one of

${ }^{2}$ See Minchin, p. 160: "The fact that gametes and pronuclei tend to unite proves that in all cases there must be intrinsic differences between them which stimulate them to do so." Coulter says on page 26:

"The gametes are alike in appearance, but that they are not alike in fact is evidenced by their pairing and mutual attraction." A similar idea is expressed by Collin (1909): "But this invisible difference be- 
general validity, it of course requires one to hold that the two chromosomes which unite at their mating within the cell are sexually diverse.

But it hardly appears possible to argue on general physical grounds that attraction and union imply diversity; two masses of substance of identical physical and chemical constitution show the attraction called gravitation; they tend to come together and unite. Adhesion occurs between bodies of like character, and in general it is not clear that all attraction of bodies must be due to chemical or physical diversities between them. ${ }^{3}$ It appears therefore that we should look for further evidence before holding that union is itself an evidence of sex diversity. If the two things that unite are characteristically diverse, the difference must show itself in other ways, so that we must rely on other tests to determine whether the diversity exists. Is there any evidence that the two chromosomes that unite in mating are sexually diverse?

In Vorticella and its relatives, as we have seen, the two individuals that mate are diverse (Figures 33 and 34). But curiously, these two-the "male" and "female"-are formed, in some species at least, by the division of a single ordinary fixed individual into a male and a female individual. The ordinary fixed individuals before this division do not mate,-

tween the two mating cells is necessary a priori, and theoretically required. For it would not be intelligible that two identical cells coming in contact should affect one another, stimulate one another out of the state of repose and cause the complicated sexual processes to begin" (p. 376).

${ }^{3}$ It is doubtless possible that all physical attraction between bodies is due to their make-up of the two opposite kinds of electrons, so that in this sense attraction may be due to diversity. But this is diversity within the bodies: the two masses, as such, may have the same constitution (including these diversities) and still attract. That is, the attraction of the two cells or individuals that mate does not a priori imply any greater diversity between them than that between two leaden bullets that gravitate toward one another. 
neither with one another nor with any males that may be present. So the ordinary individual is neither male nor female; it is apparently non-sexual or neutral. After it has divided to produce male and female, the female resembles externally the neutral individual, but differs from it in that it may mate. If it has no opportunity to mate, it may return to the neutral condition, and may then again divide to produce males and females. These facts have been worked out in Opercularia by Enriques (1907). In some other Vorticellidæ a single ordinary individual may divide into four or eight males. It is asserted that sometimes the small motile "males" mate together; and that similarly sometimes two fixed "females" mate; but these things are uncertain. There is still much to be learned as to sex diversity in this most interesting group of the Vorticellidx.

In some infusoria the two individuals that mate are alike before mating, but become changed and diverse during the mating process. This is inevitable in some infusoria, since the organisms are unsymmetrical, and if their mouth surfaces are to be brought together, either the two must take different positions, or one or both must become altered in structure. This will be evident from Figure 38.

Enriques (1908) studied this matter thoroughly in Chilodon. In this animal the mouth lies to the left on the ventral surface, so that when the two individuals are placed side by side, the mouth of the right-hand individual lies at the surface of contact, but the mouth of the left-hand individual is directed away from the surface of contact (Figure 38). But in the early stages of the process of mating, the mouth of this left-hand individual moves along the ventral surface over to the right, - so as to meet the mouth of the other individual which does not change its position (Figure 39). The anterior end of the left individual likewise be- 


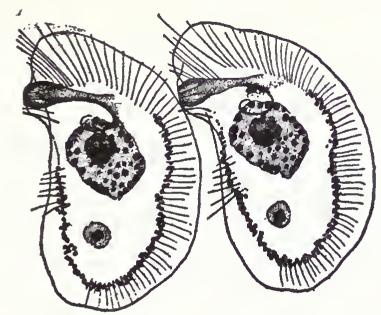

Figure 38. Chilodon. Two individuals placed side by side, showing that the mouths, lying at the left side, will not come in contact in conjugation without some alteration of position. Based on a figure by Enriques, 1908.

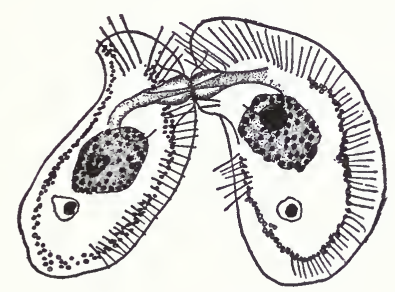

Figure 39. Conjugation of Chilodon. The mouth of the left-hand individual has moved over to the right, to meet that of the right-hand individual. After Enriques, 1908 (but reversed in position).

comes bent, in such a way as to shorten the animal, so that when the two members of a pair are measured, the left member is shorter than the right-hand one. The result of all this is that the two mates have become diverse. Enriques holds this to be the first stage in the production of sex differences; that the right-hand individual corresponds to a female, the left-hand one to a male. He believes that in other species the difference thus produced in the mating process 
lasts and is inherited, so that the two sexes are distinguishable even before mating occurs. There is no evidence that such inheritance occurs. This notion of the origin of sex of course implies that there is no underlying general physiological difference that makes sex; sex would be a mere matter of the external differences. If we believe that there is such a general physiological difference, then we could hardly hold that these differences produced during mating are sex diversities, unless we believe that they are guided by and coincide with the previously existing physiological differences. It appears probable that the diversities between the two mating individuals in Chilodon are mere transient alterations, with no lasting consequences.

So far we have dealt with the differences, or lack of differences, between the two individuals that mate. But the sex diversities reach to the more intimate structures; to the nuclei. In Paramecium (Figure 40), each individual produces a larger half nucleus that is passive, like the female; a smaller half nucleus, which is active, seeking out and uniting with the passive half nucleus of the other mate, and thus playing the part of the male. What is the difference between these two half nuclei produced by a single individual,- the "male" and "female" half nuclei? Here we have the problem of the nature of sex diversity brought to a point; if we could answer this question for these two half nuclei, we should know the nature of sex.

The differences that we observe are the following (see Figure 40 and Figure 49, H). The "male" half nucelus is a little smaller; it is usually nearer to the surface of union of the two mates; it separates from the "female" half nucleus of the same individual; it moves toward and across the surface of union; it refuses to unite with the other "male" half nucleus which it meets and passes on its way; but it does 

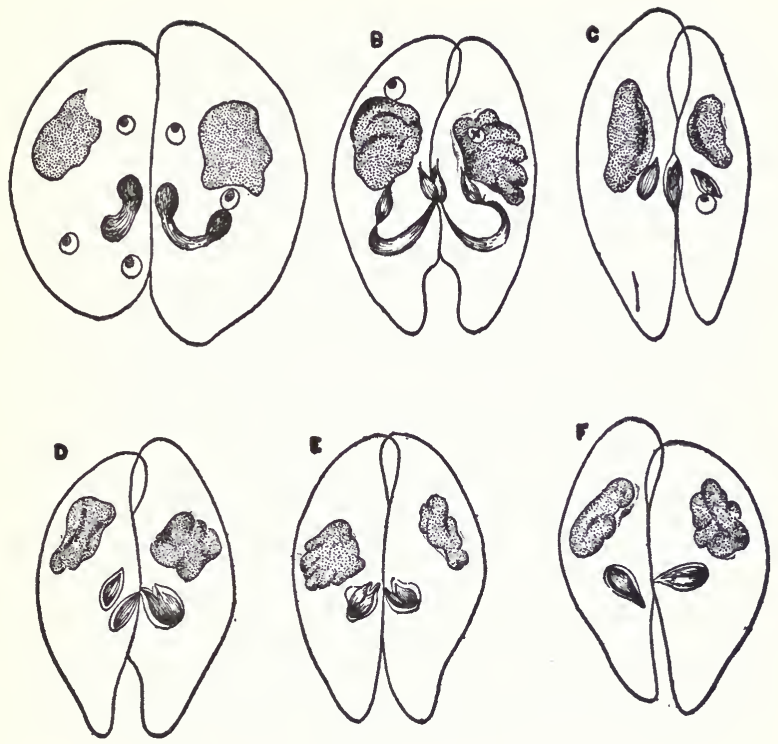

Figure 40. The exchange of the half nuclei in Paramecium caudatum, after Maupas, 1889. The spindle-shaped bodies are the half nuclei. A. The micronucleus of each member of the pair is dividing into two halves, which are still connected by a band. The upper half in each case is the "male" or "migratory" half nucleus. B. The two migratory half nuclei are passing one another at the surface of separation of the two individuals. (Each is still connected by a long band with the other half (stationary) of the nucleus from which it came.) C. The two migratory half nuclei are close together, but have now completely lost their connection with the stationary halves. D. The migratory halves have nearly separated, each passing into the body of the opposite individual. E. The migratory and stationary half nuclei have come in contact. F. The migratory and stationary half nuclei have almost united. 
unite with the "female" half nucleus of the other individual. The "female" half nucleus is a little larger; it simply remains quiet, finally uniting with the "male" half nucleus which migrates to it.

The problem of sex comes, in perhaps its sharpest form in the question: What makes the two "male" half nuclei refuse to unite when they come in contact, while the "male" and "female" do unite?

One answer to all these questions is that given by Maupas (1889). According to this, there is no physiological difference between the two half nuclei; their difference of behavior is a mere result of their accidental difference of position. The migratory half does not move through any peculiarity of its own; the fact is merely that the half nearest the surface of separation is seized by the movements of the surrounding cytoplasm and carried over into the other mate. The fact that the two migratory halves do not unite is again merely due to their being pulled along by the cytoplasm. Save for this activity of the cytoplasm, the two migratory half nuclei could just as well unite with each other as with the inactive half nuclei. According to this way of looking at the matter, there is no sex difference between these two half nuclei; indeed, sex has no meaning, save as a name for certain external peculiarities.

This is one possible opinion on the matter; it rests upon, or results in, the general view that there is no general underlying peculiarity that constitutes sex diversity; and that sexuality is not a general characteristic of living things.

The other possible view is that the difference in appearance and behavior of the two half-nuclei is a consequence of an underlying physiological difference that makes sex. In support of this view there are urged certain facts observed by a number of later investigators. One is that it is not true 
that the half nucleus nearest the surface of separation of the two mates is always the one that moves over into the other mate; on the contrary, sometimes one of the nuclei farther from this surface is the one that thus becomes the migratory half nucleus. ${ }^{1}$ Further, the actual smaller size of the migratory half nucleus, observed in Didinium by Prandtl (1906), and in Paramecium by Calkins and Cull (1907), is held to imply an intrinsic difference between the two. According to this way of looking at the matter, one of the half nuclei is male, the other female, in virtue of their diverse chemical make-up. Such a view goes with the general theory that sex in a matter of fundamental physiological diversity, not a mere name for certain external peculiarities.

Keeping these two contrasted opinions in mind, certain facts as to what occurs in these organisms are of much interest. The theory of the need for periodic unions; the theory of rejuvenescence through such unions, is based, as we have seen, on the notion that the two nuclei have been developing in opposite directions,--one toward "maleness," the other toward "femaleness," till an unbalanced condition is reached; union is then required to restore the balance.

The situation in Paramecium and other infusoria seems almost to reduce this idea to an absurdity as an explanation of a necessity for periodic mating. For here the two nuclei that are assumed to have been developing in opposite directions,- the "male" and "female" half nuclei,-have in fact been developing continuously together, in the same micronucleus of the same individual! It is only immediately before the union of "male" and "female" parts in the mating that the united "male" and "female" parts have separated.

${ }^{1}$ Calkins and Cull, 1907, p. 393; Prandtl, 1906, p. 246; Collin, 1909, p. 359 , etc. 
There is no point to the male element's separating from the female element, to immediately unite with another female element, if the whole rationale of the process is merely to bring maleness and femaleness together; they needed but to remain where they were!

From the fact that "male" and "female" of one individual separate, and immediately unite with "female" and "male" respectively of another individual, it might seem that the essential point is not the mere union of male and female, but the union of parts of diverse individuals, and many have held this to be the case. But here again we meet a set of extraordinary facts which make this way of looking at the matter as difficult as the other. In many organisms the unions that occur are between half nuclei of the same individual, or even of the same cell; this is the process that is becoming familiar under the name autogamy.

A typical striking case of this union of half nuclei from the same cell is seen in the small parasitic flagellate in-
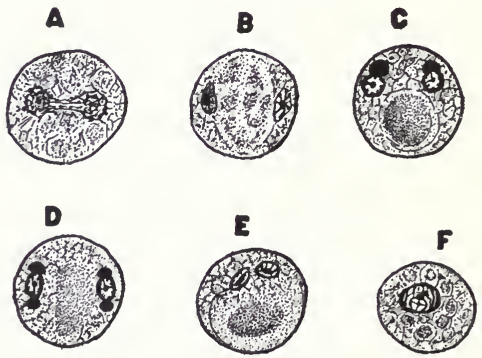

Figure 41. Mating of two halves of the nucleus of same cell (autogamy) in the cyst of Triclomastix. A, division of the nucleus; $B$, the two nuclei separated by a large mass of reserve food matter; $C$, first "reduction" division of each nucleus; D, second reduction division; E, the two nuclei approach each other; F, they unite into one. After Prowazek from Hartmann, 1909. 
fusorian Trichomastix, found in lizards. The flagellate forms a small round cyst (Figure 41), in which the single nucleus divides into two (A), the two nuclei coming to lie one on each side of a large mass of reserve nutritive material. Then each nucleus divides twice unequally, giving off two small nuclei (Figure 41, C and D). These two small nuclei, commonly known as reduction nuclei, are absorbed and disappear. The remaining two nuclei, which we can now, for reasons to be clearly seen later, call half nuclei, move toward each other $(\mathrm{E})$, and finally unite $(\mathrm{F})$. The process of mating or of fertilization is now finished.

Here again we have a case in which the two half nuclei that unite have been developing continuously together, in a single nucleus; they separate, then (after two more divisions) reunite. And in this case, it is the same two nuclei that have separated that come together again,- - save for the fact that each has divided off two small nuclei.

The same sort of thing occurs in many lower organisms.
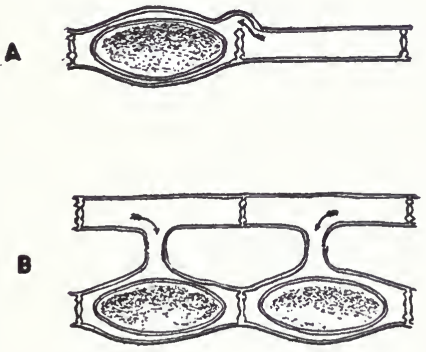

Figure 42. Two methods of conjugation in Spirogyra, after Walton, 1915. A, conjugation between adjoining cells of the same filament. The contents of the cell to the right have passed into the other cell and united with its contents. B, conjugation between the cells of different filaments. From the filament above, the cell contents have passed into the cells of the filament below. 
Sometimes, as in the case just described, it is two nuclei of the same cell that reunite. In other cases it is half nuclei from two adjoining cells of the same organism; this happens in the alga Spirogyra (Figure 42). Or again, two branches of the same plant may unite, with union of nuclei (Figure 43). Or again, two cells that have been formed by the division of a single cell may mate; this happens in Paramecium aurelia at times, as well as in other infusoria, and in algæ. We find every possible transition, from the union of two half nuclei of a single cell (Figure 41) to the fertilization of one individual by another quite unrelated to it.

In all these cases in which the two half nuclei that unite have been developing together in a single nucleus, evidently
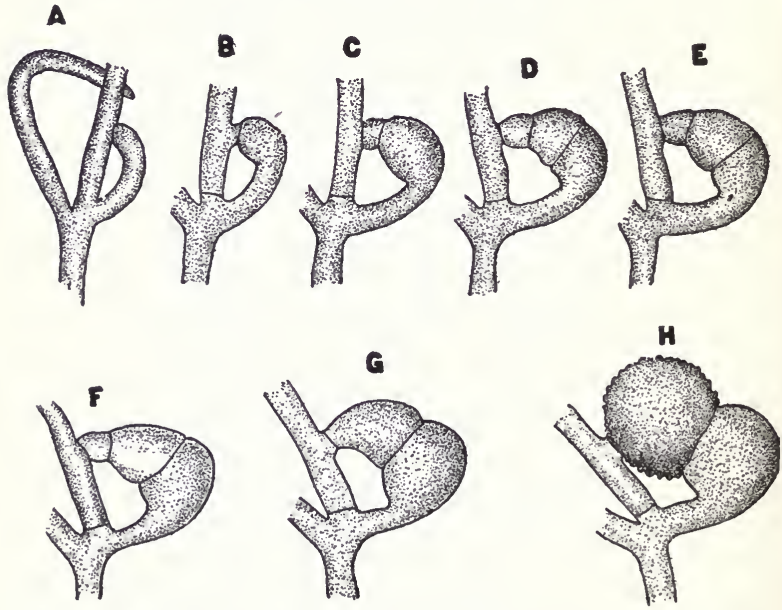

Figure 43. Process of conjugation of two branches of the same plant, in the mould Zygorhynchus. A to $H$, successive stages, leading to the formation of the dark zygospore in $\mathrm{H}$. After Blakeslee, 1913. 
we cannot explain the process as due to the gradually divergent development of two nuclei, one in the male direction, the other in the female direction, till they have become so diverse as to be unbalanced, and so to require reunion. And there is no ground on this basis for any rejuvenescence to be produced by the union; for the male and female parts that become united were already in union before the separation and reunion occurred.

A modified form of this notion is held by some students, a form so modified as to be almost if not quite empty. In such a case of reunion of two half nuclei of a single cell as we see in Figure 41, as well as in all other cases, it is maintained that the two half nuclei have become diverse, in the divisions that have just occurred,--one retaining more of the kinetic or male characteristics, the other more of the vegetative or female characteristics; and that this is the reason why the two now unite. That is, after the two half nuclei have separated, this theory if correct gives a ground for their reuniting. But it gives no ground at all for the fact that the organism periodically goes through this whole process, of separating off two half nuclei, which then again unite,-since what is accomplished by their union was already existent before the process occurred. And it gives no ground for expecting any rejuvenescence or other marked physiological result from mating. That is, it gives no explanation for periodic mating such as is given for periodic taking of food, when we show that it is the taking of food that makes possible the activities and growth of organisms. It is therefore not surprising that in a paper maintaining this theory, Hartmann (1909) concludes with the statement that he thinks it most improbable that this will turn out to be the full explanation of the matter.

On the whole, it appears that in Paramecium and many 
other infusoria, both mates of a pair play the part of both "male" and "female," so that diversity of sex cannot be given as ground for the union of the two individuals. Further, there is no clear evidence that the two uniting half nuclei are diverse in any generally characteristic way; indeed on the whole the facts perhaps agree best with the view that there is no underlying sexual difference in the uniting half nuclei; that indeed in many of these lower organisms there is no such thing as sex diversity.

And this too appears the natural conclusion with relation to that ultimate act of mating, the union of the chromosomes. In many organisms this does not occur at once after the mating of the individuals or the germ cells (though in some, particularly in the flies, it does). Usually after the germ cells unite, the chromosomes remain without mating for many cell generations, through which the fertilized egg develops into the body of a new individual. It is only when the germ cells of this new individual are ripening for their next mating that the chromosomes within each cell mate. At this time they form a set of structures that in some organisms show many diversities of size and form (Figure 29). But the mating is not between the most diverse individuals. On the contrary, we find as a rule that each chromosome has found as its mate one precisely like itself,-so that the group was really composed of sets of two individuals of the same size and form, and it is these two that mate. Exceptionally two chromosomes of diverse size or form may mate, but this is only when a similar mate is lacking, and there is evidence that such mating between chromosomes of diverse size or form is not so intimate as that between chromosomes of similar structure. All the indications are that in this ultimate act of mating the union is between structures that are 
alike, not structures that are characteristically diverse. ${ }^{4}$ The theory of two essentially diverse substances, male and female, cannot be applied in any form to the mating of the chromosomes.

But if similarity between the parts that mate is the original and elementary condition, how does it happen that in other organisms, and indeed in most, we do find a diversity of sex? Why do we find that as a rule in higher organisms a small germ cell unites with a larger one?

This has often been conceived as a special case of division of labor. It is necessary for movement to take place in order that union shall occur; it is also necessary that there shall be a certain amount of food or stored up energy for the beginning of development in the new organism. Hence it appears conceivable that variations should arise among the originally equivalent cells, - such that one set would become more active, while the other would store up more food. This of course could not occur without a corresponding change in the underlying chemical processes, but the difference in sex would according to this view not be coextensive with life. It would be a difference that has arisen in evolution, just as the difference between two races of Difflugia has arisen. In Paramecium and related organisms, according to this view, the difference has not arisen.

What chiefly raise doubt as to the correctness of this idea are the cases in which there is a physiological sex diversity where no structural diversity can be scen. But there exist

- The chromosomes are structures that perpetuate themselves from generation to generation, by division, as Protozoa do. It is not difficult to so arrange breeding experiments that two offspring of the same chromosome shall in a later generation be found in different sexes and mate together, just as we may cause two offspring of the same Paramecium to breed together. This again would be difficult to reconcile with the idea that the two mating chromosomes must be sexually diverse. 
equally cases in which by any physiological test that can be applied, there is no diversity of sex.

The only doctrine of sex diversity that appears to offer possibilities of general application is one that holds, not that there is always a definite positive characteristic for each sex, but only that there is some relative difference between the two mates, one of them being somewhat more developed in a certain direction than the other. It would not be inconsistent, on this view, if a given individual or cell or chromosome could play the part of male in some matings; of female in others,- depending on the relative development of the two mates. But such a doctrine is certainly not established; and for the mating of the chromosomes it has not a particle of evidence.

It is most important not to commit the error common in evolutionary thought, of identifying the question of the primitive nature of a phenomenon with that of its physiological significance where it is developed in a marked degree. The question whether all organisms have sex has little to do with the question as to what the nature and results of sex are, in organisms that are divided into two sexes. In such organisms - in man for example-the fundamental chemical processes that make up life are in the two sexes diverse in every cell of the body, for the nuclei on which these processes depend are diverse. There is no ground therefore for supposing that the characteristic differences in the activities of the two sexes are mere matters of different education or environment. 


\section{VI}

What are the Results of Mating? Rejuvenescence and Mating. Heredity and Variation, and Mating.

W ${ }^{\mathrm{E}}$ have so far tried to judge of the nature and effects of mating chiefly by examining the organisms or parts that unite, and the processes that occur in union. Now we turn to the study by observation and experiment of the effects of mating. Does mating actually cause rejuvenescence, in the sense of an increased vigor and vitality? The chief definite criterion for such increased vigor that has been proposed is, that after mating, reproduction should take place more energetically than before. In the Protozoa, for example, old age is held to show itself by a decrease in the rate of multiplication; rejuvenescence by an increase. This is the theory which Calkins in recent times has made peculiarly his own. "As with the metazoön so with the aggregate of Protozoa cells, we note a period of youth characterized by active cell proliferation; this in both groups of organisms is followed by the gradual loss of the division energy accompanied by morphological changes in type of the cells preliminary to conjugation and fertilization and to the renewal of vitality by this means" (Calkins, 1906, p. 232). This appears to be what is commonly understood by the theory of rejuvenescence.

How can we find out whether this theory is correct? Evidently the direct method is. to take a stock of infusoria, such as Paramecium, and allow it to multiply till it gets to the 
stage in which it is ready to conjugate. Then permit part to conjugate, while the rest are not allowed to do so, and compare the rate of reproduction in the two sets, under identical conditions. Will those that have been allowed to conjugate reproduce more rapidly than the others?

This experiment was first performed by Richard Hertwig (1889). At the beginning of conjugation in Paramecium he separated some of the pairs before the process had been accomplished, while others he allowed to complete the mating processes; he then compared the rate of fission in the two sets. Calkins in 1901 similarly compared the rate of fission in a single line derived from an ex-conjugant with the non-conjugant stock from which it came (the experiment is described in full by Calkins and Gregory, 1913). I carried out the same experiment on a very large scale in 1913, employing large numbers of lines of both non-conjugant and conjugant origin, and repeating the experiment many times. Mast (1917) has recently repeated the experiment with a number of lines of Didinium, and Calkins (1919) has still more recently carried it out with Uroleptus.

Furthermore, Maupas (1888 and 1889) made extensive and thorough researches on the same question in many kinds of infusoria, by somewhat different methods. He did not separate pairs that were beginning to mate, as was done in the other experiments, but compared the rate of fission soon after conjugation with the rate after many generations had elapsed since conjugation.

All these experiments except those which have been reported by Calkins, gave concordant results, unfavorable to the idea that conjugation increases the rate of fission. Maupas found that there was in none of the cases he studied an increase of fission rate after conjugation; nor a decrease as 
the time elapsed since conjugation became greater. Hertwig found that the descendants of those that had conjugated actually multiplied more slowly than the descendants of their companions that were not allowed to conjugate. Mast found that in Didinium the rate of fission after conjugation is a little slower for a short time; but soon the rate becomes equal in those that have and those that have not lately conjugated. In my own extensive experiments, with large numbers of lines in both classes, the average fission rate was uniformly less in the lines that had recently conjugated than in those that had not. Conjugation, as we shall see, caused much variation between the diverse lines, in fission rate as well as in other hereditary characters. At the upper extreme of the variation sometimes a conjugant line exceeded in its fission rate the non-conjugant lines. But in most of the lines the rate after conjugation was decidedly less than before; uniformly the average of the conjugant lines was much below that of the non-conjugant ones. ${ }^{1}$

In Calkins' experiments, on the other hand, the lines which had conjugated showed a more rapid fission rate than those which had not. In his experiment on Paramecium in 1901, in which a single line derived from an ex-conjugant was compared with the non-conjugant stock from which it had come, the ex-conjugant line divided 376 times in nine months, while the non-conjugant lines produced but 277 generations. It may be noted that another conjugant line, derived from the mate of the line that produced 376 generations, died out completely after the production of but 11 generations. This illustrates the fact shown on a large scale in my own experiments, that conjugation brings about great variation in

${ }^{1}$ The actual comparative rates in certain cases will be given later (page 158). 
vitality and reproductive power. The single line retained by Calkins was clearly one of the rare extreme variants, with a high fission rate.

In Calkins' recent work on Uroleptus, ${ }^{2}$ it is set forth that in this organism the effect of conjugation is uniformly to increase the rate of fission. The fission rate, however, is given in terms of averages of sets of five lines; such averages were always higher for the sets that had recently conjugated than for those that had not. But it is not possible to discover from them whether the fission rate was higher in all the lines of conjugants or whether as in my own experiments the rate was lower in some of the conjugant lines, higher in the others. Obviously it was higher in the majority at least of the conjugated lines.

The fact that some of the lines derived from ex-conjugants may exceed in their fission rate the non-conjugant stocks, whether this is rare, as in Paramecium and apparently in most other infusoria, or common, as in Uroleptus, is unquestionably a fact of great significance; this renders it possible to still maintain, as Calkins does, that mating at least sometimes produces rejuvenescence, in the sense of increased vigor of reproduction. We shall discuss this matter farther in dealing with the question of the production of inherited variation by conjugation. But if we base our conclusions on the usual or the average effect of conjugation, we are forced to conclude that in most cases it does not increase the rate of multiplication, but rather it decreases this rate.

It is not generally realized that the work of Maupas and of Richard Hertwig, on whose authority the theories of rejuvenescence by conjugation largely rest, was squarely opposed to the idea that this rejuvenescence manifests itself by an increase of reproductive vigor. It is worth while to ${ }^{2}$ Calkins, G. N., Proc. Nat. Acad., April, 1919. 
emphasize this point, for upon it an extraordinary error has become widespread even among expert biologists. ${ }^{3}$

Maupas rejected emphatically the idea that aging shows itself in a decrease of the rate of reproduction and that conjugation restores this declining rate. He asserted positively that no such decline occurred before conjugation and that after conjugation reproduction was not more rapid than before, and he based these statements on the results of careful experimentation. Richard Hertwig's experiments led to the same result and induced him to subscribe completely to Maupas' assertions on this point. The matter is important, since the doctrine that conjugation restores the declining fission rate depends largely on their supposed support of it. I therefore give in their own words statements of the results of their experiments, and of the conclusions they drew from these results.

Maupas sums ip the prevailing mistaken doctrine of his time on this matter in words which well fit the present conditions:

"It has been affirmed that the faculty of reproducing by fission in the ciliates was modified by conjugation, and that the principal effect of this sexual act was to reënforce and accelerate this reproduction. The ciliates, it was held, immediately after conjugation multiply more rapidly than they do at a later stage. This opinion has become current, and one finds it reproduced in Memoirs and in general treatises, as if it were a definitely established truth" (1888, p. 254-255).

Maupas then proceeds to show that the evidence on which

Thus, investigators so experienced as Woodruff and Erdmann in a recent paper (1914) contrast my own results on this point with "the view of Bütschli, Maupas, Hertwig and others," asserting that according to the latter, after mating "all the processes of the cell, including reproduction, proceed with greater vigor." 
this theory had been based amounted to nothing. Next he sets forth that in his own records of fissions in different infusoria, beginning in a number of cases with animals that had just conjugated, there is no indication of a greater rate of fission after conjugation. He says of the fissions :

"They succeed one another uniformly, modified only by the changes of temperature. I did not remain content with this one experiment. I isolated other ex-conjugants of Stylonychia pustulata, of Onychodromus grandis, of Euplotes patella, of Paramecium aurelia, of Leucophrys patula. I followed day by day the successive generations of their descendants, during periods of from fifteen days to two months. In none of these species did I see any difference in the succession of fissions. Whether they had conjugated lately or a long time ago, all the individuals acted in the same way" (1888, p. 255-256).

Maupas sums up the matter in a later paper as follows: "I have asserted, besides, that this power of multiplication is maintained regularly and uniformly during the entire life cycle; that there is no gradual weakening of this power from the first generation after conjugation up to the return of a new period of maturity. In other words, I deny that the infusoria after conjugation have a more energetic reproduction than they have at a later period" (1889, p. 504).

Certainly this is sufficiently explicit not to be misunderstood! It is because Maupas' papers, with their hundreds of pages of text filled with observational and experimental details, make hard reading, that it is possible for mistaken ideas of his results to become prevalent.

Richard Hertwig (1889) found in his experiment, to his surprise (as will anyone that tries it), not only that the 
Paramecia which had conjugated did not reproduce faster, but that, on the contrary, it was those that had not been allowed to conjugate that multiplied more rapidly. Speaking of the theory that conjugation increases the energy of reproduction, Hertwig says :

"The grounds on which this theory is based have already been combated by Maupas; he showed by extended experiments that the power of multiplication of an infusorian is neither decreased before conjugation nor increased after it. . . I I am compelled to say that Maupas was entirely right" (1889, p. 222).

What then did Maupas and Hertwig mean by holding that conjugation does nevertheless rejuvenate?

Maupas' theory is not easy to seize or to state in experimental terms. He believed that without conjugation the organisms became deformed and structurally degenerate, although their power of reproduction remained unimpaired; at last they died. Conjugation, he believed, if it occurs before degeneration has become evident, prevents the process of degeneration. His own experiments showed him, as we shall see, that after observable degeneration has begun conjugation does not remedy it. The only test for Maupas' theory is formed by such experiments as those of Woodruff, in which it is shown that even without conjugation the animals live indefinitely and do not degenerate.

Hertwig (1889), taking the bull by the horns, held with relation to Paramecium, that an increase in the rate of reproduction was one of the symptoms of degeneration; that conjugation in restoring the balance decreased the rapidity of fission. It is extraordinary that two such contradictory views can be held of what conjugation does, as are these two - Hertwig on the one hand maintaining that conjugation decreased the rate of reproduction, Calkins on the other that it increases it-both under the name of rejuvenescence! 
Hertwig has developed later a general theory of mating, rejuvenescence and kindred matters; a theory which is at once extremely special and extremely indefinite. It is based upon ideas of a necessary proportion in quantity between the mass of the nucleus and that of the cytoplasm. As growth occurs the cytoplasm increases faster than the nucleus, so that after a time the nucleus is too small in proportion to the amount of cytoplasm. This brings about in some way a sudden increase in the growth of the nucleus, and this in turn causes division of the cell. In the course of many cell generations, through irregularities in division, and through the action of various agents, the nucleus may become too large in proportion to the cytoplasm. Enlargement of the nucleus has been found to occur in cold; in hunger; when the animals are overfed; and in various other conditions. In such conditions the animals do not divide frequently, and this is attributed by Hertwig's theory to the disproportionate size of the nuclei. Such conditions of large nuclei and slow reproduction, with other pathological symptoms, are spoken of as states of "depression." Such depression can be remedied, if it has not gone too far, in various ways, -by change of temperature, of food, and the like; the essential thing that then happens, according to this view, is that the nucleus reduces its size, by throwing out substance into the cytoplasm, or otherwise. But if depression has gone too far for this, it can be overcome only by a deep-seated reorganization of the nucleus. Such a deep-seated reorganization occurs in the processes connected with mating; hence, it is held, these processes restore the normal balance of nucleus and cytoplasm; the depression disappears, and the organisms continue to live and multiply.

Such a theory appears artificial and based upon superficial features. When one considers the complex chemical 
processes that lie at the foundation of life, it is difficult to believe that mere relations of proportionate quantity of two things so complex as nucleus and cytoplasm, both having the power of assimilation and growth, is at the bottom of these matters of life, death and reproduction. As Dobell (1909) expresses it, one would almost "as soon argue that grey hairs are the cause of old age in man" as to hold that increase in size of the nuclei is the cause of degeneration and death in cells. Furthermore, this theory gives absolutely no ground for the chief feature of mating,-the fact that it is a mating of two nuclei, not a mere reorganization. And like the other theories of rejuvenescence, its validity depends finally on whether mating actually does restore vigor, vitality and reproductive power to depressed organisms. Most of such theories have proceeded on the ground that if one can show that degeneration occurs without conjugation, then it must follow that mating remedies the degeneration. The real test lies in observing whether mating actually does remedy the degeneration. If it does not, the probability becomes strong that the degeneration is simply a pathological result of bad conditions. The evidence is becoming overwhelming that this is the case; that to avoid degeneration, it is merely necessary to avoid the bad conditions. The work of Woodruff, set forth in our first lecture, goes far to demonstrate this.

Hertwig's own test of the matter in Paramecium was, as we have seen, opposed to the idea that mating increases the vigor of reproduction; Maupas' extensive work gave the same result, and my own still more extensive experiments led to the same conclusion. Only Calkins' experiments with Uroleptus stand in the way of asserting this to be the general rule in infusoria.

Certainly no clear case has been made out for rejuvenes- 
cence as a general result of mating. Indeed it is only fair to say that most of the positive evidence is against rejuvenescence of any sort. In Paramecium, not only do those that have conjugated multiply less rapidly than before, but a great many of them die after conjugation,-although without conjugation they live vigorously. Many others are weak and sickly, multiplying but little. Still further, conjugation produces a great number of abnormalities and monstrosities, such as do not occur without mating (see Figure 44). In many other infusoria all or nearly all those that have conjugated die, or completely cease multiplying. Maupas made many investigations giving such results. He allowed two unrelated cultures of Stylonychia to become degenerated, then let them conjugate together; according to the theory this ought to have rejuvenated them. But it did not; they all died. He found that even in wild Stylonychias conjugation rather usually results in death; later students have confirmed this. In his experiments, Maupas found that the individuals that have conjugated all die in Spirostomum, Climacostomum and Didinium. In Leucophrys he found that a large proportion of those that mate die. It used to be supposed that such results were due to the fact that the animals had become so degenerate that even conjugation could no longer save them. But we now know that under proper conditions, without conjugation they would (in most cases at least) live and multiply vigorously, while after conjugation they are weak or die.

Such facts are most extraordinary; they are difficult to explain on any theory. A priori it appears that the replacement of the old active nucleus by the reserve nucleus, must tend to give rejuvenescence in conjugation, just as when it occurs without conjugation. But this tendency is in the majority of cases completely overwhelmed and done away with by other features of mating; by the physiological diff- 
culties of the complex process; and as we shall try to show, by frequent incompatibilities between the parts united.

In rare lines in Paramecium, and other infusoria, and commonly in Uroleptus, according to Calkins, all these difficulties are overcome, so that the replacement of the worn nucleus by the reserve has the same effect in renewing vitality that it has when its occurs without mating. There is thus far no evidence either from Calkins' experiment with Uroleptus, or from any others, that the mating, as distinguished from the replacement of the macronucleus by the micronucleus, produces rejuvenescence. Calkins finds that in Uioleptus this replacement without conjugation restores vitality as it does with conjugation.

What then are the distinctive results of mating, as compared with that replacement which occurs without mating? On this the theory of rejuvenescence has not thus far cast light. The thing to do under such circumstances is to drop for the time any definite preconceived theory, and examine with care the facts; sometimes these carry a theory of their own! Just what differences can we find between individuals that have mated and those that have not?

In our examination of the question of diversity of sex in Paramecium (Lecture 5), we mentioned a difference that was found in that organism. After mating, the two individuals and their off spring have become more alike than the two stocks were before mating. This, as before remarked, is not unnatural, for each individual after mating is, if it may be so expressed, partly made up from the other individual. In all of the offspring of each of the mates, half the nucleus has come from the other mate. It is therefore not surprising that the two sets of offspring are much alike; they are all children of the same family; they are as closely related as are the brothers and sisters of a human family.

Here then we have in these lower organisms a distinctive 
visible difference made by mating, and it is the same sort of result that is produced by mating in higher organisms. In the latter in consequence of mating, off spring are produced which are more alike than are individuals taken at random; this is likewise what happens in Paramecium.

In higher organisms we say that this result is an example of heredity. As we are accustomed to think of it, the offspring in a family inherit from both their parents; they resemble both the parents; and this appeals to us as a reason why they should resemble each other. Now, whether the offspring of the two members of a pair of Paramecia resemble also their parents has not been directly shown; it is a difficult matter to get at; what we know is that they do show a marked resemblance to each other. But this matter of resemblance between the external features of parent and offspring is in reality a totally inadequate basis for a conception of heredity. In the study of heredity in higher organisms, we often discover that the off spring inherit characteristics from the parents which the parents do not show, -as when rabbits with white hair are born from parents with brown hair; or when two white fowls produce black chicks. In such cases the black color of the chicks is inherited from the white parents just as truly and in just the same way as occurs when black parents produce black chicks. The only consistent meaning we can give to heredity is this: heredity is the appearance of peculiarities in the offspring that are due to the peculiarities of the germinal material they obtained from their parents. And we know that white fowls do frequently transmit to their off spring germ cells of such a character that the offspring must be black; such cases are recognized as clear examples of Mendelian inheritance. By biparental inheritance we mean merely this: the peculiarities of the given individual, taken as a 
whole, are due to its origin from the united germinal material from two parents; if the individual had been derived from but one parent, it would have shown other peculiarities. Thus when two kinds of white flowers produce by their union plants with red flowers, this is an example of biparental inheritance (and one of not uncommon kind),-for the red color would not have occurred if the two parents had not united, as for example in reproduction through cuttings.

Now, the resemblance between the offspring of the two members of a pair of infusoria is due to the fact that they have received germinal material (the two halves of their micronuclei) from both parents. It is therefore an example of biparental inheritance.

I have given this brief exposition of what heredity means, and particularly of what biparental inheritance means, because to persons not familiar with experimental work in heredity, it appears surprising that we should speak of biparental inheritance when we haven't proved that the offspring resemble their two parents; this objection has been made by Dobell (1914). But as a matter of fact we speak of inheritance equally when we know that the off spring do not resemble their parents,-provided their peculiarities are due to the germinal material derived from their parents. The other significance for heredity would exclude a large proportion of the best known cases of Mendelian inheritance.

We find then that in these Protozoa, as in higher organisms, mating results in inheritance from both parents. In what respects does such inheritance occur? Our knowledge on this point is scanty; until recently it was confined to certain characteristics of Paramecium; to this has now been added important knowledge of these matters in Chlamydomonas. This is one of the lines of work in which there is 
most room for acquiring valuable knowledge; but it is extremely difficult to carry out the necessary experiments. The facts for Paramecium are as follows :

1: in Paramecium, biparental inheritance occurs with reference to size; after conjugation the two sets of progeny are more alike in size than before (See Jennings and Lashley, 1913a).

(2) It occurs also with relation to rate of fission. Here it is often very striking. If the descendants of one member of a given pair multiply rapidly, the descendants of the other member are likely to multiply rapidly also; if one set multiply slowly, so also as a rule does the other set. One or two examples (from Jennings and Lashley, 1913) of this are perhaps worth while. Calling the two members of a given pair $a$ and $b$, the numbers of fissions for four successive periods of ten days each were for several pairs, all under the same conditions:

\begin{tabular}{|c|c|c|c|c|}
\hline Pair & & & & Total \\
\hline 41 & 10 & 11 & 10 & $12=43$ \\
\hline 41 & 10 & 10 & 12 & $15=47$ \\
\hline & 5 & 5 & 5 & $4=19$ \\
\hline 47 & 5 & 7 & 4 & $5=21$ \\
\hline & 9 & 7 & 8 & $8=32$ \\
\hline 9 & 8 & 6 & 6 & $9=29$ \\
\hline 108 & 11 & 11 & 13 & $13=48$ \\
\hline & 11 & 13 & 12 & $13=49$ \\
\hline
\end{tabular}

As you see, both members of pair 41 divide rapidly; of pair 47 slowly; of 9 , at an intermediate rate; while in pair 108 both members divide still more rapidly than in pair 41. This condition of affairs is typical.

(3) There is a similar resemblance between the offspring of the two members in respect to vigor and vitality. Sometimes the descendants of one pair are weak and the stock gradually dies out. When this happens, the family derived 
from the other member is likely to be weak and die out also. This was studied with very great thoroughness, and a strong tendency to likeness in this respect was found (Jennings and Lashley, 1913).

(4) A fourth respect in which the off spring of the two that have mated tend to be alike is in certain structural abnormalities that often occur (Stocking, 1915). Such abnormalities are shown in Figure 44: Frequently these abnormalities are hereditary in the family derived from one member of a pair; when this is the case, the family derived from the other member often shows such abnormalities also,much more frequently than is the case when its mate is normal.

In all respects in which the matter has been examined, therefore, mating tends to make the families derived from the two members of a pair more alike than they would have been without it. Conjugation produces biparental inheritance, just as fertilization does in higher organisms.

In Chlamydomonas, Pascher ${ }^{4}$ has recently succeeded in studying the results of conjugation with respect to characters of a more tangible sort than those that we were forced to use in Paramecium. Two species of Chlamydomonas differed in form and structure in the way shown in Figure 43a, A and B. In conjugation these organisms unite completely, forming a cyst. The cyst formed by the conjugation of two individuals like $\mathbf{A}$ has no membrane and is covered with pyramidal elevations, as shown at AA, while that formed by two like $B$ is smooth and surrounded by thick membranes (BB). When $\mathbf{A}$ conjugates with $\mathbf{B}$, the resulting cyst is intermediate between the two pure forms, as shown at $\mathrm{AB}$; there is a thin layer of membranes, and the surface is covered with low rounded elevations.

'Pascher, A., Ber. d. Deutsch. Bot. Ges., 1916 and 1918. 

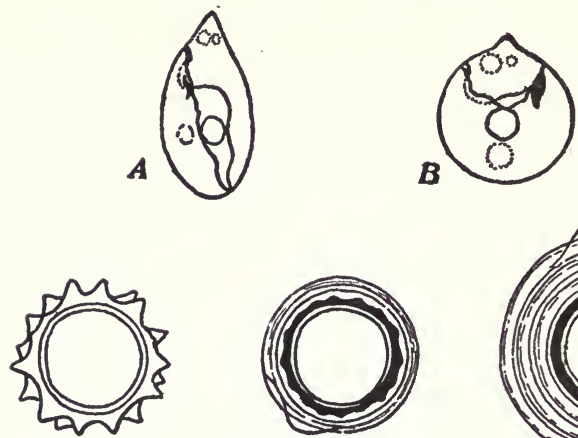

A A

$A B$

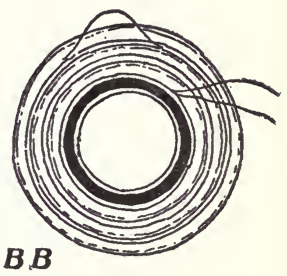

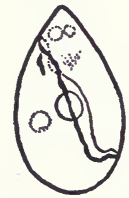

8.

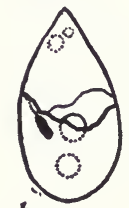

$\boldsymbol{b}$
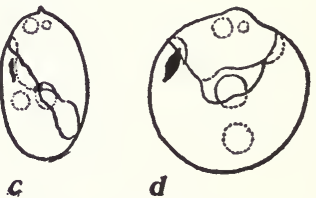

Figure $43 a$. Biparental inheritance and the production of diversity by conjugation, in Chlamydomonas, after Pascher (1916), from Hartmann. ${ }^{1}$ A and B, the two parental species. AA, cyst formed by conjugation of two individuals such as $\mathrm{A}$. BB, cyst formed by conjugation of $\mathrm{B}$. AB, cyst formed by conjugation of $\mathrm{A}$ with $\mathrm{B}$. $a, b, c, d$, the four types of free living individuals resulting from the conjugation of $A$ and $\mathbf{B}$.

In this cyst the reducing divisions occur (Lecture VII), and it divides into four free-swimming individuals which when the conjugation was between two similar parents, are like those parents. These free individuals now reproduce by fission in the usual way.

When the cyst had been formed by conjugation of the two diverse kinds of parents $\mathbf{A}$ and $\mathbf{B}$, the progeny show a num-

${ }^{1}$ Hartmann, M., Zeitschr, f. Ind. Abst., September, 1918. 
ber of diverse combinations of the characters of the two parents, as shown in Figure 43a, $a, b, c, d$. Pascher (1918) gives a table of the various combinations found in the four sets of individuals in comparison with the characteristics of the parents $\mathrm{A}$ and $\mathrm{B}$, as follows:

\begin{tabular}{|c|c|c|c|c|c|}
\hline $\begin{array}{l}\text { Parent A } \\
\text { Parent B }\end{array}$ & $\begin{array}{c}\text { Form } \\
\text { pear-shaped } \\
\text { spherical }\end{array}$ & $\begin{array}{c}\text { Membrane } \\
\text { delicate } \\
\text { coarse }\end{array}$ & $\begin{array}{r}\text { Papilla } \\
\text { none } \\
\text { present }\end{array}$ & $\begin{array}{c}\text { Chromato } \\
\text { lateral } \\
\text { basal }\end{array}$ & $\begin{array}{c}\text { phore Eye spot } \\
\text { linear } \\
\text { broad }\end{array}$ \\
\hline $\begin{array}{cc}\text { Offspring } & a \\
6 & b \\
6 & c \\
\ll & d\end{array}$ & $\begin{array}{l}\text { pear-shaped } \\
\text { pear-shaped } \\
\text { ellipsoidal } \\
\text { spherical }\end{array}$ & $\begin{array}{l}\text { delicate } \\
\text { delicate } \\
\text { coarse } \\
\text { coarse }\end{array}$ & $\begin{array}{l}\text { none } \\
\text { none } \\
\text { present } \\
\text { present }\end{array}$ & $\begin{array}{c}\text { lateral } \\
\text { basal } \\
\text { lateral } \\
\text { basal }\end{array}$ & $\begin{array}{l}\text { linear } \\
\text { broad } \\
\text { linear } \\
\text { broad }\end{array}$ \\
\hline
\end{tabular}

The offspring $a$ and $d$ resemble very closely the two parents respectively, while the types $b$ and $c$ show characters of both the parental forms. This is the same sort of result which we find in the Mendelian inheritance of higher organisms. It is a demonstrated fact, therefore, that in the Protozoa, as in higher forms, conjugation results in biparental inheritance.

Observe also that besides producing resemblance of parents to progeny or of progeny to one another, conjugation causes stocks or races or families to arise which are diverse in their hereditary characters from either of those from which they took origin. This is evident in Chlamydomonas; $b$ and $c$ show diverse combinations from those found in either of the parents, and these diversities are perpetuated in their propagation by fission. In Paramecium, from a single race-all having the same hereditary charactersthere may arise by conjugation many races with diverse hereditary characters.

What this means will best be illustrated from an actual experiment (from Jennings, 1913). We begin with a set of individuals all alike, all derived by fission from a single parent. We study their rate of fission; taking 174 separate 
lines of descent, we find it to be extremely uniform. For periods of 21 days we find the number of fissions of the first 24 lines to be those given under "Non-conjugants," $a$ and $b$, in the following table. (The two individuals, $a$ and $b$, of the non-conjugants, had begun to mate, but were separated before the mating process occurred.) In these 24 lines the number of fissions in 21 days varied only from 21 to 26 ; it is on the whole very uniform. One finds little or no indication of inherited differences between the families; they run very evenly.

Now we allow a large number of the individuals to conjugate, then follow the rate of fission for 88 lines derived from these mates. We find that there are now great differences between the separate families with respect to fission rate. In the table the two last columns, headed "Conjugants," give the number of fissions for 24 lines descended from pairs of conjugants, for the same 21 days as for those that have not conjugated. The two sets (Nonconjugants and Conjugants) were kept throughout under the same conditions.

\section{Table}

Comparative fission numbers in 21 days of Conjugants and Nonconjugants derived from a single race $\mathbf{E}$ (from Tables 34 and 35; Jennings, 1913). In the non-conjugants, $a$ and $b$ represent two individuals that had begun to mate, but were separated before mating was accomplished. In the conjugants, $a$ and $b$ are the two mates from one pair.

\begin{tabular}{ccccc}
\multicolumn{2}{c}{ Non-conjugants } & \multicolumn{2}{c}{ Conjugants } \\
Pair & a & b & 2 & b \\
1 & 25 & 23 & 30 & 29 \\
2 & 24 & 23 & 25 & 24 \\
3 & 24 & 24 & 29 & 28 \\
4 & 24 & 25 & 10 & 9 \\
5 & 23 & 24 & 8 & 10 \\
6 & 22 & 25 & 24 & 24 \\
7 & 22 & 26 & 29 & 28 \\
8 & 21 & 25 & 25 & 24 \\
9 & 24 & 25 & 30 & 27 \\
10 & 22 & 26 & 26 & 26 \\
11 & 23 & 24 & 28 & 27 \\
12 & 25 & 24 & 11 & 9
\end{tabular}


In the descendants of those that have conjugated the number of fissions in 21 days varies from 8 to 30 , as compared with 21 to 26 in the others. There are now strongly marked differences between the different families that have descended from those that have conjugated. It is interesting to compare the records of fissions by two-day periods for a considerable time in such families derived from different ex-conjugants, Typical examples are as follows:

$\begin{array}{rllllllllllll}\begin{array}{c}\text { Family } \\ 1 \text { ax }\end{array} & 0 & 2 & 2 & 2 & 3 & 4 & 4 & 4 & 3 & 3 & 3 & 3=33 \\ 6 \text { ax } & 0 & 2 & 1 & 1 & 1 & 1 & 1 & 2 & 2 & 2 & 1 & 2=16 \\ 9 \text { ax } & 1 & 2 & 2 & 3 & 4 & 4 & 3 & 3 & 3 & 3 & 3 & 4=35 \\ 27 \text { ax } & 1 & 3 & 1 & 1 & 2 & 2 & 2 & 2 & 2 & 2 & 3 & 1=22 \\ 15 \text { ax } & 2 & 2 & 2 & 2 & 1 & 2 & 0 & 1 & 0 & 1 & 0 & 0=13\end{array}$

All these families were kept under the same conditions, yet they consistently differ in their fission rates throughout the entire period. They have become hereditarily diverse in this respect, as a result of conjugation, for all were derived, before conjugation, from the same original parent, and all had the same rates of fission. Conjugation has produced from a single stock a number of hereditarily diverse stocks. The same thing was demonstrated in a number of extensive experiments.

Another respect in which conjugation increases the hereditary differentiation is in the matter of abnormalities. If we begin with a set of the animals that are all quite normal, showing no inherited abnormalities, and allow them to conjugate, we often find that many of the families produced have hereditary abnormalities, while others have none. Such hereditary abnormalities, produced as a result of conjugation, are shown in Figure 44. In work by Dr. Stocking (1915), of 450 families descended from animals that have conjugated, 262, or more than half, showed hereditary 


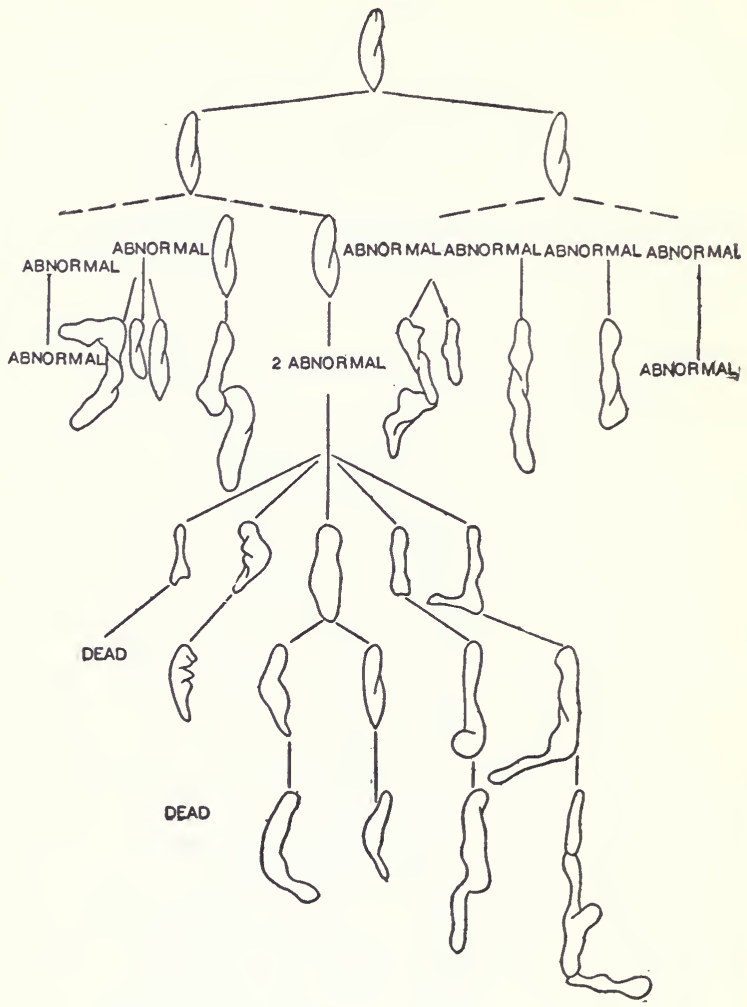

Figure 44. A family of Paramecium caudatum, descended from an ex-conjugant, and showing hereditary abnormalities. Besides the individuals figured, other abnormal branches of the family are indicated by the lines with the word "abnormal." After Stocking, 1915.

abnormalities,-although none were present before conjugation. 
There is evidence also that conjugation causes hereditary differentations to appear with respect to size, but this matter has not been so precisely studied as it deserves to be.

Calkins and Gregory (1913) observed similar hereditary variations in fission rate and vitality arising after conjugating. They give some evidence that hereditarily different families may arise from the first two divisions of one of the members of a pair. During conjugation, as we have seen, new macronuclei are produced from the reserve micronucleus (see Figure 30). The first four of these are produced within the individual that has conjugated, and before reproduction takes place. Then by two divisions these are distributed to the four progeny. According to the results of Calkins and Gregory, the four individuals receiving the four macronuclei thus produced may become diverse; probably, it would seem, through the diversity of these four nuclei. In later divisions such diversities do not appear, according to their account; at least they say that each of the lines produced remains "true to its type for many months at least." I believe that this matter requires further study, but according to the results of Calkins and Gregory, these inherited differences are strietly the result of conjugation, just as are those shown in my own work.

We find therefore that in all the hereditary characters in which the matter has been studied, conjugation gives rise to inherited differences; in other words, diverse stocks arise as a result of conjugation. Such work needs to be greatly extended; all we know on the matter is based on Chlamydomonas and Paramecium, mainly the latter, and its characters are not so favorable for such work as might perhaps be found. But such work is extremely difficult. ${ }^{5}$

${ }^{8}$ Certain work of Mast (1917), bearing on this point, in the infusorian Didinium, will be taken up in our next lecture. 
We must now revert for a moment to the relation of this production of diverse stocks through mating to the problem of rejuvenescence. Among the characters in which mating induces hereditary diversities is the rate of fission. This has been illustrated on page 158 .

Now, as we have seen, if we take all the lines that have conjugated and average them, we find that their average fission rate is somewhat less than the average rate of those that have not conjugated. Thus in a very extensive experiment in which 69 lines derived from conjugants were compared for a period of three weeks with 145 lines derived from non-conjugants, the daily fission rate of the conjugants averaged 1.097 , that of the non-conjugants 1.144 (Jennings, 1913, p. 349). For the period of 21 days the average number of fissions in each line was for the descendants of the conjugants 23.041 ; for the descendants of the non-conjugants, 24.034. When we study the separate lines we find that those descended from the non-conjugants all show nearly the same number of fissions; the slowest line had 18 fissions, the fastest 28, in 21 days. But the lines descended from the conjugants differ greatly among themselves; the slowest line has for the twenty-one days only 9 fissions, while the most rapid one has 31. That is, mating has caused much hereditary diversity in the fission rate of the descendants of the conjugants, and those at one extreme of the variation have a higher fission rate than those descended from the non-conjugants. Out of the descendants of 56 ex-conjugants, in this experiment, 9 produced families with a higher fission rate than any of the families descended from the 130 non-conjugants. On the other hand, 9 of the families produced from the exconjugants had a lower fission rate than the low family (with 18 fissions) produced from the non-conjugants. 
The descendants of the ex-conjugants do not by any means always show some families that exceed the fission rate in the descendants of the non-conjugants. In nine extensive cultures (described in my paper of 1913, page 364) in which this matter was studied, in but three did the maximum for the conjugants exceed that for the non-conjugants, while in all cases the minimum and the average for the conjugants were below those for the non-conjugants.

We have set forth the relations found in Paramecium, the organism most thoroughly studied from this point of view. In Uroleptus, according to Calkins, those that have conjugated usually show an increased rate of reproduction. The production among the combinations resulting from mating, of families with an increased vigor of reproduction (usual in some infusoria, occasional or rare in others) of course justifies the statement that in those cases conjugation has caused rejuvenescence, as maintained by Calkins. Consistency with the facts compels one, however, to say that while mating sometimes produces rejuvenescence, in most of the individuals that mate, it produces, in the majority of the known species, not rejuvenescence, but its opposite, the animals being less vigorous and multiplying less rapidly than would have been the case if they had not mated.

But why should mating produce rejuvenescence in some cases and not in others? In other words, why has mating such diverse hereditary results in different cases? This question we shall take up in our next lecture; here we may indicate merely the general nature of the answer which we shall reach. We should expect conjugation to give rejuvenescence just as endomixis does, since in it too the old macronucleus is replaced by the unused micronucleus. But in conjugation there are likewise produced new combinations of the parental characters. Some of these new combinations are vigorous; 
others are weak. In the former rejuvenescence appears; in the latter it does not.

What conditions bring about mating in these organisms? It is of interest to bring the facts observed on this point into relation with those we have above set forth as to the results of mating. Many generations pass in which the individuals do not mate; then at a certain time some or many or all of them mate. Is this the result of the internal changes that have been in slow progress, so that conjugation occurs when a condition of ripeness or of need for it has been reached? This was the idea held by many who believed the life of these creatures to go in cycles of youth and age; mating occurs, it was held, in a certain period of the cycle. Or, on the other hand, is mating rather brought on by certain external conditions? Much study has been devoted to these questions.

In Paramecium and many other infusoria it has been observed that an epidemic of mating usually occurs when a period of high nutrition, resulting in rapid multiplication, is followed by a period of scarcity of food. Artificial cultures of hay or other vegetation in the laboratory often go through such a cycle; at first bacteria are abundant and the infusoria flourish on them; then fermentative changes go so far that the appropriate bacteria are scarce; the infusoria become thin, and begin to mate. It is easy to furnish these conditions if from a flourishing hay culture we remove a watch glass of the water with many of the infusoria and allow it to stand, without any of the vegetable material, for 24 hours. As the bacteria become scarce the infusoria conjugate. This method has been used practically by many investigators in order to obtain matings for study.

If part of the animals are kept supplied with abundant 
food, while the others are subjected to a scarcity, the latter conjugate, while the former do not. This is true even when all the individuals are derived from the same single original parent. It is clear therefore that there is no imperious necessity for conjugation at a particular period in the life history ; and that a period of scarcity following a period of abundance will induce conjugation when it would otherwise not occur. In some of the writer's experiments the off spring of a single individual were divided into two sets; one set was caused in this way to go through conjugation four times in succession (the mates at any conjugation being the offspring of the mates at the preceding conjugation); while the other set during the entire period did not conjugate at all. In some races of Paramecium aurelia, after a pair had mated their descendants in the fourth generation were thus caused to mate again; while in other members of the same stock hundreds of generations passed without conjugation.

Clearly therefore the occurrence of conjugation is in large measure the result of special external conditions. This matter has been much studied of late by Enriques and by his pupil $Z$ weibaum. They have found that conjugation is favored by special conditions in particular species of infusoria; thus in Colpoda steinii conjugation occurs when the layer of water in which they are has become a thin film (Enriques 1907), - as happens just before a pool is dried up by evaporation. In Cryptochilum Enriques (1910) discovered that certain salts greatly favor conjugation. In Paramecium caudatum Zweibaum (1912) has determined with great precision the conditions that induce mating. He finds that after the infusoria have been subjected to a period of scarcity of food for five to six weeks, if the nutritive conditions are suddenly changed for the worse, and at the same time certain salts are present in proper concentration, 
the animals will always conjugate. The salts found to favor conjugation were the compounds of sodium and other metals with chlorine, bromine and other halogens. Aluminium chloride was found to be the most favorable of those studied.

Thus Zweibaum and Enriques hold that the environmental conditions, past or present, fully determine whether conjugation shall occur. It is true that of two stocks side by side under the same present conditions, one may conjugate, the other not; but this in their opinion is due to the fact that one has been subjected to a long period of scarcity of food, while the other has not. That is, while the two stocks may indeed at a given time differ in their internal conditions, this difference is not a matter of diversity in the life cycle, comparable to youth, maturity and age, but is merely a result of the different external conditions under which they have been living. The occurrence of conjugation is, they hold, in last analysis, determined by external conditions.

There is certainly a large measure of truth in this conclusion, though it is perhaps not yet completely established in its absolute form. The question may be asked why it is necessary that the period of scarcity of food should last so long as five to six weeks before it induces conjugation? Does this perhaps indicate that a certain number of generations after a foregoing conjugation are necessary before a new mating can occur ${ }^{1}$ Zweibaum's experiments need to be repeated in such a way that after one conjugation a new culture is produced from an ex-conjugant, and the period of time determined (or if possible the number of generations) that must necessarily elapse before a new conjugation can be induced. Zweibaum did not determine whether an intervening conjugation does away with the accumulated effects of continued scarcity of food, so that the organisms must

${ }^{1}$ This question has already been raised by Erdmann (1913). 
again be subjected to five or six weeks' scarcity before they will again conjugate. This is really the essential point; for if this turns out to be the case, then evidently the length of time since a previous conjugation is one of the things that determine whether conjugation shall now occur.

But independently of this doubtful point, it is clear that mating at a particular period is not required independently of the outer conditions, for Paramecium aurelia will live indefinitely (over 6000 generations) without conjugation (Woodruff), yet may be induced to conjugate if the required outer conditions are supplied (Woodruff, 1914); and in some stocks of this species a second conjugation may be induced in the fourth generation after a previous conjugation (Jennings, 1910, p. 286). Certainly by far the largest part is played by external conditions (past or present) in producing conjugation.

The conditions under which mating occurs (sudden scarcity of food and the like) are conditions which are distinctly unfavorable to the life of the organisms. Some species of infusoria respond to such conditions by becoming encysted; they transform into a small sphere, protected by an outer coating; and in this state they can withstand conditions that would otherwise destroy them. Some other Protozoa respond by first conjugating, then encysting. In others, such as Paramecium, there is only conjugation, without encystment. But as we have seen, conjugation results in the production of many diverse stocks, some of which are more resistant to given conditions than others. It appears that some of the stocks so produced may be able to survive the unfavorable conditions which induced conjugation, although (as observation shows) most of them die out if the conditions are not altered for the better. Later generations would therefore all be derived from the most vigorous and 
resistant stocks resulting from the new combinations formed in mating. There is ground for believing that in nature this process occurs on a large scale.

Looking back over what has been found out as to the effects of mating, the general picture is as follows: It has been shown that infusoria may live and multiply indefinitely without conjugation (Woodruff, Enriques). It has been shown that at intervals the old active macronucleus is replaced by a part of the reserve micronucleus. These things demonstrate that the mating process (as distinguished from the replacement process) is not necessary for continued life and vigor. They appear to disprove any theory of sexuality that maintains that there must for continued life be a periodic reunion of two substances, male and female, which inevitably become separated as a result of life and development. Rejuvenescence is through the replacement of used parts by unused ones, and this occurs without mating, although it may occur at mating also. The distinctive contribution of the mating itself is something else.

Investigation shows that mating produces two very striking results: (1) It causes the off spring of the two individuals that have conjugated to become more alike; it produces biparental inheritance. (2) It causes the different families produced by different pairs to be hereditarily diverse in many respects; and this even when all the parents come from a single ancestor and are hereditarily alike.

Do we find anything of this sort elsewhere in organisms? Consideration brings to light the fact that this is precisely what results from mating in higher organisms; we call the detailed working out of these results Mendelian heredity. In heredity in higher organisms, the offspring produced by any pair resemble each other more than they do other individuals; they show biparental inheritance. Furthermore, 
the offspring produced by the different germ cells of even the same pair of parents are hereditarily diverse; a single pair of parents may, in plants or certain animals, produce in Mendelian inheritance hundreds of hereditarily different kinds of offspring. The only reason why this may not occur in the highest animals and man is that in these cases relatively few of the possible combinations develop, since but few offspring are produced.

In these respects, therefore, mating does the same thing in the Protozoa that it does in the higher organisms. In both it brings biparental inheritance and the production of hereditarily diverse stocks. 


\section{VII}

How Does Mating Bring About Both Biparental Inheritance and Diversity in Hereditary Characters? What Effect Has Mating on the Stock as a Whole? Does It Increase Variation? Does It Decrease Variation? What Is Its Relation to Evolution?

I $\mathrm{N}$ our last chapter we showed that mating produces biparental inheritance, as well as diversity of inherited characteristics, in lower as well as in higher organisms. How are these results brought about? How does it happen that the offspring of the two members of a pair on the whole resemble each other, yet are hereditarily diverse?

The main outlines of the way this is brought about are well known. Each parent hands on bodily to the offspring, through the germ cells, certain packets of chemicals. Since these are directly transmitted from parent to offspring, while the later characters are secondarily derived from them, we may call these packets of chemicals the primary hereditary characters. These packets are present in each animal in a certain definite number, stored within the nucleus; they are called chromosomes (see Figures 29, 31, 32). Individuals which get different sets of packets from their parents develop differently even under the same outer conditions; that is, they show different hereditary characteristics.

This arrangement of the primary hereditary charactersthe chemicals that determine the hereditary peculiarities- 
into packets is fundamental for an understanding of how heredity occurs; it is this that directly brings about all the peculiar phenomena that are called Mendelian inheritance. And this is a typical illustration of the part played by structure and arrangement in organisms; it demonstrates that a chemical study alone, omitting arrangement of the chemicals, can never lead to comprehension of what occurs. The point is that when two or more chemicals are in a certain space, it makes all the difference in the world as to what happens, whether the two substances are in separate bottles, or merely poured together. To neglect this fact in organisms is as fatal to understanding them as it would be to try to comprehend what occurs in a chemical laboratory without realizing that the different chemicals are kept in separate containers. The nucleus of the cell is a chemical laboratory containing diverse chemicals in separate packets. At times substances come out of these packets, intermingle, and therefore react. It is their reactions with each other, and with external conditions, in an orderly way, that bring about growth and the development into a structure with diverse organs. The packets are shifted about and distributed in certain ways at the time of mating and fertilization, and it is the rules of their distribution that are what we call the rules or laws of inheritance.

We know that in any organism these packets of chemicals are present in a definite arrangement. We know that each larger packet or chromosome is a chain or group of connected small packets (Figure 29, E, F), and that the different chromosomes present in a nucleus are diverse. Their number is definite in any individual, and they are so constituted as to form a set of diverse pairs (Figure 29; Figure $45 \mathrm{~B}$ ); (though sometimes there is a single package or chromosome that is not paired with another). 
When mating is to occur we know that these paired packages of each nucleus separate into two groups, each group containing one member of each pair (Figure 45, B, C, D). These two groups are then separated by cell division into different cells. Each of these cells therefore contains a group with half the number of packages that were present in the parent nucleus. It is such cells with half the original number of packages or chromosomes in their nuclei that form the germ cells, - the two cells that are to
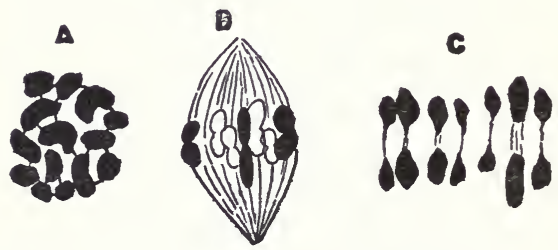

4

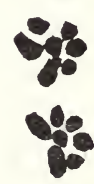

Figure 45. The separation of the two groups of paired chromosomes into different germ cells, in the insect Nezara hilaris, after Wilson, 1911. A, the 14 chromosomes in a single cell, before the germ cells are formed. $B$, the 14 gathered into 7 pairs. $C$, the members of the seven pairs separating as the division to form the germ cells occurs. $\mathrm{D}$, the two groups of 7 chromosomes each, in different germ cells, formed by the separation of the 14 shown in $A$ and $B$.

unite in mating (Figure 45, D). After the two half nuclei have united, of course the original number of chromosomes is restored.

As before remarked, we know that the different chromosomal packages present in a nucleus are diverse. The evidence for this is complete, but cannot be given here. Now, when these diverse packages separate into two half groups, different half groups are formed in different cases, depending on which member of any given pair goes into a given group. In this way a great number of diverse com- 
binations are formed in the half-groups derived from different nuclei, even of the same parent.

This diversity of the combinations of the primary hereditary characters in the different half nuclei is the essential point in understanding the way the later hereditary characters are distributed, so that it will be best to illustrate how it comes about. Suppose we represent the chromosomal packages in the nuclei of a particular animal by letters of the alphabet. We will indicate the diversities by giving a different letter to each chromosomal packet, and to the two members of a given pair we will give a capital letter and a small letter respectively. To illustrate the principles in simple form, we will suppose that there are but four pairs of chromosomal packages in each nucleus. That is, the chromosomes of each nucleus would be represented as follows :

\section{A B C D \\ a b c d}

Now in each parental nucleus of this kind the chromosomes separate into two groups, one member of each pair in each group. But either member of any pair can go into either group. That is, from one nucleus the two groups formed may be A B C D and a b c d; from another nucleus they are $\mathrm{A} \mathrm{B} \mathrm{c} \mathrm{D} \mathrm{and} \mathrm{a} \mathrm{b} \mathrm{C} \mathrm{d;} \mathrm{from} \mathrm{another} \mathrm{A} \mathrm{b} \mathrm{C} \mathrm{d} \mathrm{and} \mathrm{a} \mathrm{B} \mathrm{c} \mathrm{D,}$ and so on. The number of different combinations from four pairs is 16, and each occurs as frequently as any other. ${ }^{1}$

So from a number of parental nuclei, all having the same combination of packages, a large number of different combinations will be formed in the half nuclei. ${ }^{2}$

${ }^{1}$ These facts, fundamental for the understanding of the rules of inheritance, have recently been directly demonstrated for certain higher organisms, by Carothers (1917).

2The number of diverse combinations possible in the half nuclei formed from nuclei all of the same kind is $2^{\mathrm{n}}$, if $n$ is the number of diverse pairs of chromosomes present in the original nuclei. 
The offspring are finally produced by the mating of two of these half cells (or germ cells), each containing a half nucleus. A nucleus with any combination of the chromosomal packages may unite with any other. Thus we shall get in the case imagined such combinations as
A B c D
a B c d
a B C d
or
a $\mathrm{B}$ C d and the like.

The total number of different combinations produced when these were originally four pairs of different chromosomal packets is $81 .^{3}$

If the number of chromosomal pairs is greater, the number of combinations produced by mating is greatly increased. For each additional pair of chromosomes the number of possible combinations is multiplied by 3 . Where there are 24 pairs of chromosomes, as apparently in man, the number of possible diverse combinations mounts far up into the trillions.

Each one of these combinations of chromosomal packets gives a different result in heredity; a different set of hereditary characters. The result is that the progeny produced by the different germ cells differ from each other, and it becomes impossible to predict from the characteristics of the parent what will be the characteristics of particular offspring, for many diverse kinds of offspring can be produced by the same pair of parents.

These processes are best known in higher organisms. But study shows that the same things occur in the Protozoa. These matters are extremely difficult to work out in these minute creatures, and an immense amount of work remains to be done before we shall know with full details what happens

- If there were $n$ pairs of chromosomes present in the original nuclei, then by the formation and mating of germ cells in the way described, the number of different combinations producible is $3^{\mathrm{a}}$. 
in these organisms. But we find that in these, as in higher animals, there are diverse packets of chemicals, which are directly transmitted from parent to offspring, so that they constitute the primary hereditary characters. In some of the Protozoa these chromosomes are extremely minute and numerous; this is the case in the infusorian Paramecium caudatum (Figure 49). In others they are larger and present in smaller numbers, appearing much as they do in higher organisms (Figure 46). The primary hereditary characters or chromosomes are shown for a number of Protozoa in figures 46 to 50 .

In preparation for mating, and in mating itself, these chromosomes undergo the same process of reduction in number and recombination into new groups that occurs in higher organisms. In figures 46 to 50 is shown what occurs in some of the groups of Protozoa.

Examine for example figure 46, which shows the process in a protozoan belonging to the Gregarinidæ, and parasitic in the earthworm, - as described by Mulsow (1911). The chromosomal packets are in the form of eight long threads (Figure 46, A) much resembling the chromosomes of many higher animals. In ordinary multiplication by fission each of these chromosomes splits lengthwise (Figure 46, B), and half of each goes to each of the two offspring (C). In the two parents before conjugation there are as usual eight of these chromosomes, which become arranged side by side in pairs, as occurs in higher organisms. This grouping into four pairs is partly seen in figure 46, D and E. Now in the early stages of mating, a division of the nucleus occurs at which one member of each pair goes to one of the daughter nuclei, one to the other $(F, G, H)$. That is, each of the two nuclei produced now receives four entire chromosomes, in place of eight. Then in the mating, two such nuclei, each 


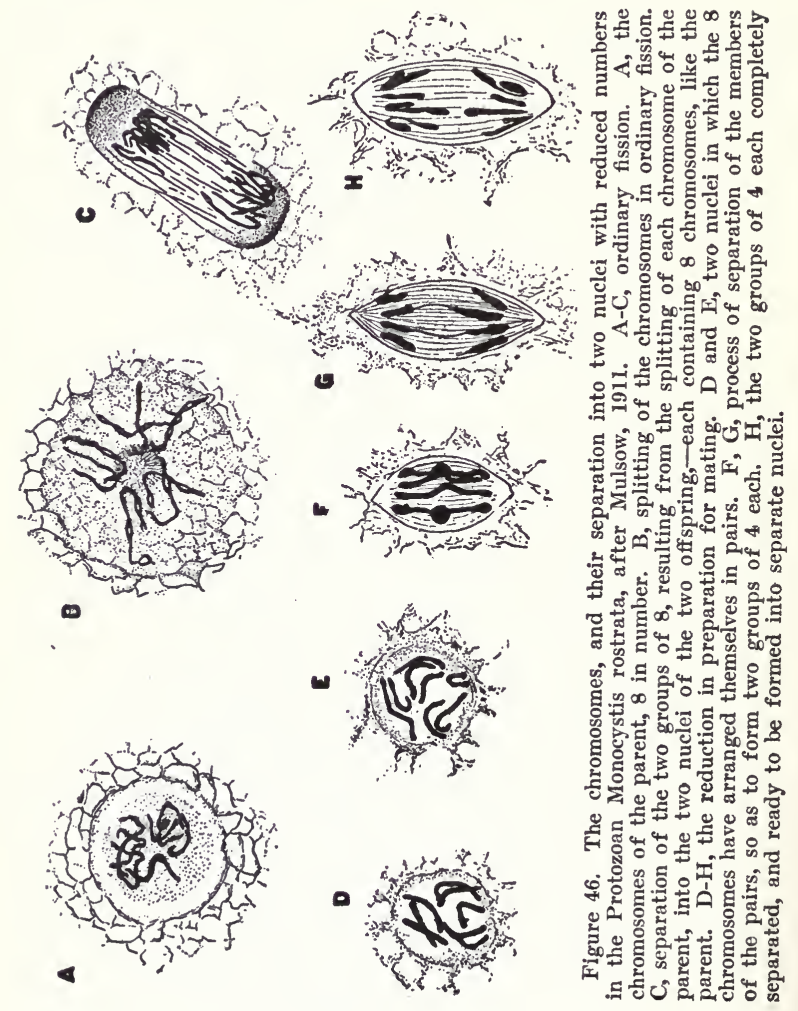


with four chromosomes, unite, forming a new nucleus with eight chromosomes. In this entire process of reducing the number to four and then, by mating, restoring it to eight, of course many different combinations of the chromosomes may arise in the different resulting individuals, in the way already set forth. The number of possible diverse combinations in this case with four pairs of chromosomes, is, as we have seen, 81 .

Reduction is better known in the ciliate infusoria than in any other group of Protozoa. To understand what happens, one must recall the fact that at the beginning of mating there are three successive divisions of the micronucleus, the third one producing the migratory and stationary half nuclei. These are indicated in Figure 35. These three divisions are commonly spoken of as the first, second and third maturation divisions; we shall employ these designations.

In the infusorian Didinium nasutum (Figure 47), according to Prandtl (1906), there are 16 minute chromosomes (A). In the first of the three maturation divisions each of these 16 chromosomes divides into 2, so that the resulting two micronuclei still have 16 chromosomes (Figure $4 \%, \mathrm{~B}$ ). But in the second division, the 16 chromosomes merely separate into two groups of $\mathbf{8}$, one group going to each of the two resulting micronuclei ( $, D, E, F)$. In the third division (G, H, I) each of the 8 chromosomes present divides into two, so that each of the two half nuclei now has 8 chromosomes. Now the migratory half nucleus from one mate passes over and unites with the stationary half nucleus of the other (Figure 47, J, K, L), so that the resulting complete nucleus now has 16 chromosomes. In the later divisions of this complete nucleus, each of the 16 chromosomes divides $(M)$, so that all the nuclei of later generations 


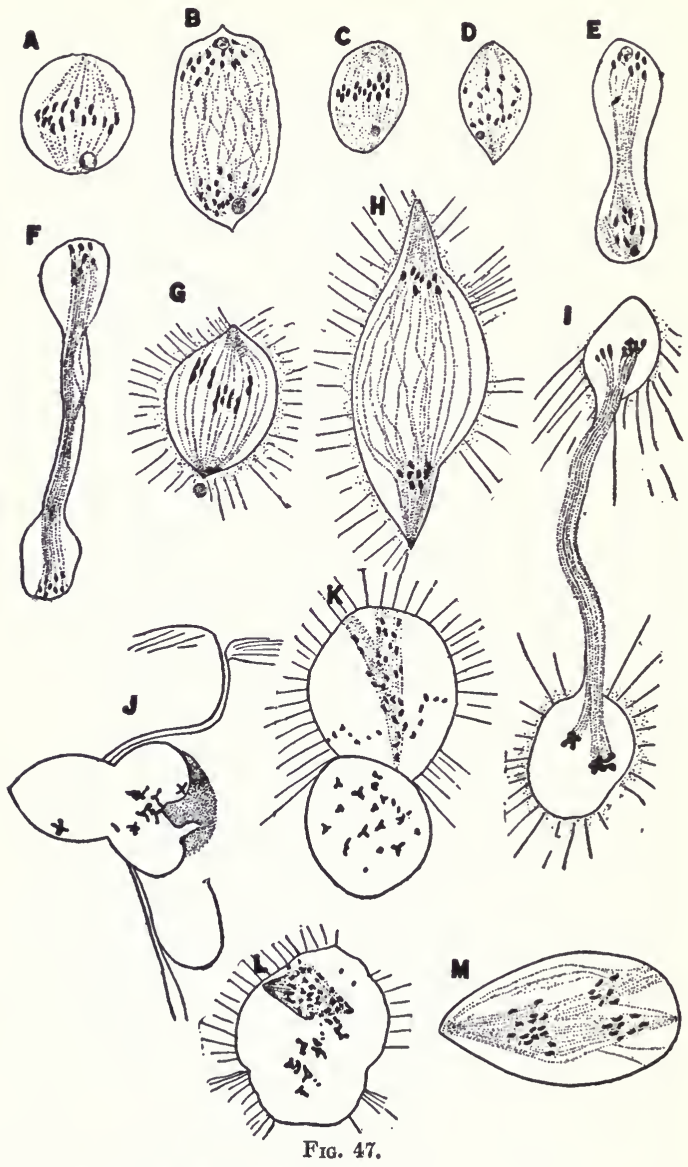

(For description see opposite page) 
Figure 47. Reduction of the number of chromosomes, and other processes in the nuclei, at conjugation in Didinium nasutum. After Prandtl, 1906.

$A$ and $B$, the first of the three divisions of the micronuclei ("first maturation division"). In A, a spindle has been formed with 16 chromosomes; in B, each chromosome has divided, so that two groups of 16 are present; one group to go to each of the two resulting micronuclei.

$\mathrm{C}, \mathrm{D}, \mathrm{E}$ and $\mathrm{F}$, the second division (the "reducing" division). C, 16 chromosomes; spindle-forming for division. $\mathrm{D}$, the 16 chromosomes beginning to separate into 2 groups. E, the 16 chromosomes have separated into 2 groups of eight each, each going to one of the two resulting micronuclei. $\mathrm{F}$, the two resulting micronuclei, each with 8 chromosomes; still united by a connecting strand from the spindle.

G, $\mathrm{H}$ and $\mathrm{I}$, the third division, which forms from a single nucleus the migratory and the stationary nucleus. $G$, each of the 8 chromosomes dividing. $\mathrm{H}$, the two groups of 8 chromosomes widely separated, to pass into the two resulting nuclei. I, the migratory nucleus (above) and the stationary nucleus (below) still joined by a connecting strand. The stationary nucleus already considerably larger than the migratory nucleus.

$J$, the migratory nucleus passing through the membrane that separates the two mated animals, into the other individual.

$\mathrm{K}$, union of the migratory and the stationary nuclei. The latter (above) is much larger than the former. L, the two nuclei almost completely united.

$M$, the first division of the nucleus formed by the union of the migratory and stationary nuclei. The union is not quite complete, so that at the right end two spindles can be seen. Each of the 16 chromosomes has divided into two, so that two groups of 16 are now present. 
have 16 chromosomes,-until another reduction occurs preparatory to another mating.

In the infusorian Anoplophrya branchiarum, which is a parasite in the blood of the fresh water crustacean Gammarus, the number of chromosomes is 6 . These are reduced to 3 before the mating (Figure 48); by the union of two at mating the number 6 is restored (Collin, 1909).

In Opercularia coarctata, a relative of Vorticella, according to Enriques (1907), the unreduced number is 16 ; the

A

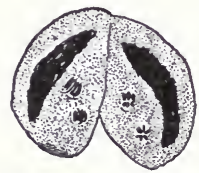

B

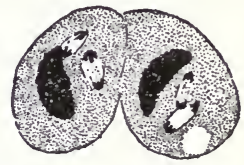

Figure 48. Conjugation and reduction in the number of chromosomes in the infusorian Anoplophrya branchiarum, after Collin, 1909. A, the two micronuclei (after the first maturation division) have each 6 small chromosomes. $\mathrm{B}$, each micronucleus dividing anew, showing the separation of the group of 6 into two groups of 3, one at each end of the spindle.

reduced number 8 . The reduction occurs at the second of the three maturation divisions; and the number 16 is restored at mating. In the infusorian Chilodon uncinatus, according to the same author (Enriques, 1908), the number of chromosomes before reduction is 4 . At the second maturation division, these are reduced to 2 , in the usual way. Mating restores the original number 4.

In Carchesium, according to Popoff (1908), the micronuclei have, before the time of mating, 16 chromosomes. At the first maturation division 8 of these go into one of the resulting half nuclei, 8 into the other. At the second and third divisions each of these 8 chromosomes divides into two, 
so that finally the two half nuclei that mate have each 8 chromosomes. The original number, 16 , is restored by the mating.

In Opalina intestinalis, a large infusorian parasitic in the alimentary canal of amphibians, the number of chromosomes during ordinary reproduction by fission is 8 . In the spring there appear small animals, which divide several times, then encyst; when they come out of the cysts they mate, two individuals completely uniting. These small individuals before mating have but 4 chromosomes in place of 8 . How the reduction is brought about is not known (Metcalf, 1909).

In the two common species of Paramecium, aurelia and caudatum, the nuclear processes at mating appear to differ considerably. They are best known in Paramecium caudatum, through the work of Calkins and Cull (1907). In this animal the matter is greatly complicated by the fact that a very large number of chromosomes is present (Figure 49). The number is so great that they cannot be counted, but Calkins and Cull estimate them at about 165 .

In individuals beginning mating, the chromosomes appear as double rods (Figure 49, A). Calkins and Cull suspect that this is due to the pairing of two chromosomes, such as we saw in Monocystis (Figure 46). At both the first and second maturation divisions these double chromosomes split lengthwise. One of these divisions therefore apparently separates the paired chromosomes (reducing the number to half in each resulting half nucleus); the other divides each chromosome lengthwise. The reduced number is apparently therefore present in the micronuclei before the third division. This third division takes place in a very different way from the other two. The chromosomes, instead of being long threads, fall into strings of granules; and each of these strings is broken transversely, at about its middle (G). 

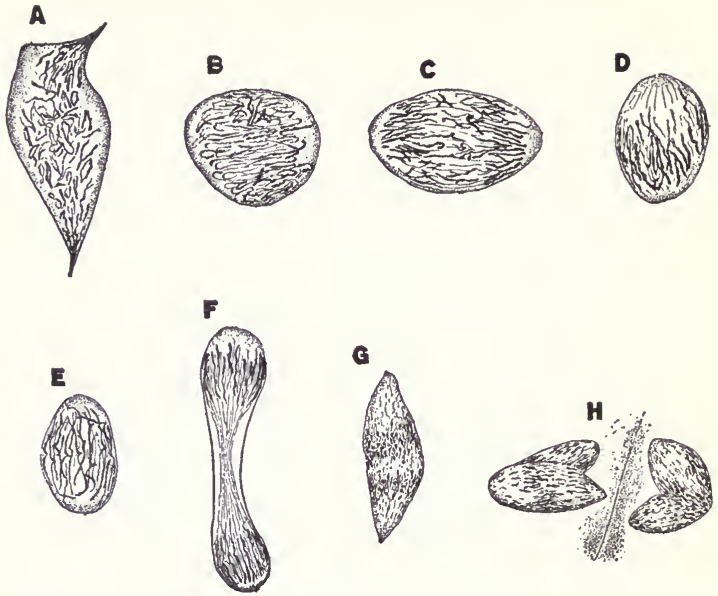

Figure 49. The chromosomes and the divisions preparatory to mating in Paramecium caudatum, after Calkins and Cull, 1907. A, B, C, first of the three maturation divisions. A, the numerous chromosomes united in pairs lengthwise (or split?). B, the two chromosomes (or halves?) separating lengthwise. C, the two chromosomes (or halves) almost separated; two groups forming. D, one of the two nuclei resulting from the first maturation division. E, F, second maturation division. E, the two halves of the chromosomes pulling apart. F, the two groups separated; the two new micronuclei united by a narrowed connecting zone. G, third division (that producing the migratory and stationary half nuclei). The chromosomes formed of rows of particles; the rows have broken in the middle and the halves are separating. $\mathbf{H}$, union of migratory and stationary half nuclei, in the two individuals. The oblique line is the surface of separation of the two mates. In each individual the migratory half nucleus is the smaller one.

Thus at this division each of the two half nuclei formed receives a half of each of the chromosomes (then present in the reduced number). The original number would of course be restored by the mating of the migratory and the stationary nuclei $(\mathrm{H})$.

Reduction is not so well known in the other groups of 
Protozoa as in the ciliate infusoria. In the gregarine Monocystis however we find a particularly beautiful example (Figure 46). In the flagellates, Schaudinn (1904) and Prowazek (1904) described a reduction from 8 chromosomes to 4 , in a number of species (Trypanosoma noctuæ, $T$. lewisi, T. brucei, and Herpetomonas). The accuracy of these accounts has been called in question.

Bott (1907) has described the reduction of the number of chromosomes in the rhizopod Pelomyxa, an animal re-
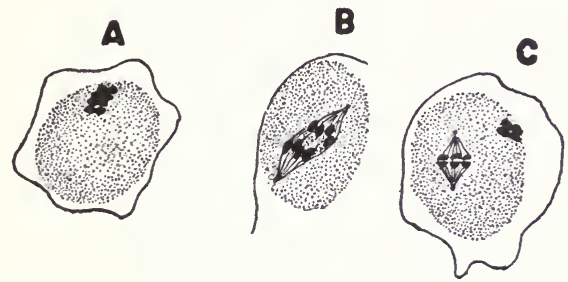

Figure 50. Reduction of the number of chromosomes before mating in the rhizopod Pelomyxa, after Bott, 1907. A, the eight oval chromosomes of the parent. B, first of the two maturation divisions; the 8 chromosomes separating into two groups of 4 . $\mathrm{C}$, the second maturation division; in the spindle to the left each of the 4 chromosomes (of which but 3 are in view) is dividing into two.

sembling a large amœba. The single animal contains many nuclei. At times these go through two divisions in succession, which may be called the maturation divisions (see Figure 50). At first there are eight oval chromosomes (A). At the first division (B), four of these go into one of the resulting cells, four into the other; the number is thus reduced. At the second division each of the four chromosomes is divided (C), so that all the nuclei produced have four chromosomes. Later each of these nuclei, along with a little cytoplasm, separates from the body of the 
mother, forming a free cell or gamete. When two of these gametes meet, they unite; thus the original number of chromosomes is restored.

Only a few of the Protozoa have been examined with sufficient thoroughness to reveal this process of reduction and recombination in the chromosomes, and in some the chromosomes are so numerous, minute and crowded that just what occurs cannot be directly determined. But the cases already worked out, scattered as they are through the different classes of the group, show that the process is one of general occurrence, here as in the higher organisms; they make it possible to recognize the occurrence of reduction even when the chromosomes cannot be counted. Just before mating, the nuclei, both in the Protozoa and in higher organisms, go through the two successive divisions (in the infusoria, owing to special conditions, three), known as the maturation divisions. It is in one of these as a rule that the reduction in number occurs, through the distribution of half the chromosomes to one nucleus, half to the other. In most cases these two (or three) divisions are distinguishable from all others by marked peculiarities connected with the reducing process; and these make it possible to recognize the reducing divisions even when the number of chromosomes cannot be counted. Whenever two (or three) peculiar divisions occur in rapid succession just before the nuclei are ready to mate, we may be practically certain that in these the reduction in the number of chromosomes has occurred. Such divisions we saw in the cases of autogamy (page 134 and Figure 41); in our examination of the preparations for mating in the infusoria (Figures 46 to 50); they occur indeed almost universally in preparation for mating. All such divisions indicate a process of reduction in number 
of chromosomes, with resulting formation of a new combination through mating.

For the Protozoa it is clear therefore that so far as the primary hereditary characters-the chromosomes-are concerned, mating is a process of producing new combinations of hereditary characters. In higher organisms we know that these primary hereditary characters are what determine also the later or secondary hereditary characters, - those that appear in the developed body. There can be no doubt that the same is true of the Protozoa. Everything indicates that in these respects mating in the Protozoa is the same sort of thing that it is in higher organisms, and that when the matter is fully studied it will be found to produce the same kind of results. Mating may be defined as the process of producing new groups of hereditary characters, primary and secondary, by combining diverse half groups from different nuclei.

But what shall be said from this point of view of the cases in which the two half nuclei are produced from a single one, and these two later unite in mating, as in the numerous cases of autogamy (Figure 41, etc.)? These cases form a difficulty for almost any other way of looking at the matter, but not for this one. For new combinations of the primary hereditary characters are formed likewise when the mating is between two nuclei that are recent products of a single one.

In all cases of such autogamy, a fact is observed which becomes of the greatest significance. After a single nucleus has divided into two, these two do not reunite at once, but there are always one or two intervening divisions. And it is these intervening divisions that bring about the reduction in number of the chromosomal packets, with the consequent formation of new combinations of the primary hereditary 
characters in mating. Thus, in Figure 41, we see that after the separation of the original nucleus into two, each of these two divides twice, giving off two very small nuclei, which are absorbed and disappear.

It is worth while to notice just how new combinations are formed in these cases in which the two nuclei that mate originally came from the same single nucleus. Let us suppose that the original single nucleus had four pairs of the chromosomal packets; these we may designate as follows:
A B C D
a $\quad$ b c c $d$

Now when this nucleus divides into two, the division is of the usual sort, in which each single packet divides into two like itself, so that each of the two nuclei produced has the same set of chromosomal packets that its parent had.

But now each of these two goes through the "reducing division," in which the set of eight divides into two sets of four each,- - one member of each pair going to each resulting set of four. Then evidently many different combinations may be formed, depending on how the members are distributed. In one nucleus the group of four will be A B C D, in another $\mathrm{A} \mathrm{B} \mathrm{C} \mathrm{d,} \mathrm{in} \mathrm{another} \mathrm{A} \mathrm{b} \mathrm{c} \mathrm{D,} \mathrm{in} \mathrm{another} \mathrm{a} \mathrm{b} \mathrm{C} \mathrm{d,}$ and so on (16 different combinations are possible). Now two of these combinations of four unite. It is practically certain that they will have different combinations; let us suppose that one contained the combination $\mathrm{A} \mathrm{b} \mathrm{c} \mathrm{D,} \mathrm{the}$ other the combination a b C d; then the nucleus formed by their union will show the combination
$A$ b c D
a b C d

That is, from a nucleus showing the combination
A B C D
a b c d 
we have by division, reduction, and reunion obtained a totally different combination.

In other cases, nuclei with the same original combination will give still different results; there are 81 diverse resultant combinations that may be produced in this way from a single nucleus having the combination of packets supposed above.

Since the facts as to the recombination and distribution of these primary hereditary characters are the same in Protozoa and in higher organisms, we may expect them to produce the same results. That is, we may expect to find Mendelian inheritance in Protozoa, when the facts are fully studied. Mendelian inheritance is nothing more nor less than a recombination and distribution of the secondary hereditary characters in the manner that the primary hereditary characters are recombined and distributed,-without doubt in consequence of this recombination and distribution of the primary characters.

There is, of course, no reason to expect such a Mendelian distribution of inherited characters among the progeny of a single individual that divides after conjugation. All such progeny will probably be alike, save for any accidental variations in the splitting of the chromosomal packets; this, as we have seen, observation shows to be the case. But if the progeny are examined from a large number of pairs coming from two diverse races that have been induced to conjugate, we may expect to find among these the typical Mendelian distribution of characters.

This may be illustrated most directly by the inheritance of the primary characters (the chromosomes); we know that the secondary hereditary characters follow the primary ones.

Let us suppose, for example, that in the two races a pair of chromosomes differ; we will say that they are black in one 
race, white in the other (see Figure 51, P). Now we know that in each individual before mating, one chromosome of each pair is gotten rid of, the half nucleus that remains containing but a single chromosome of this pair; and that this half nucleus divides to form the migratory and stationary half nuclei of that individual; so that migratory and stationary half nuclei contain the same set of chromosomes. In the one race they will, of course, therefore both contain a black chromosome, in the other a white one. When the exchange and union of half nuclei occur, the resulting nucleus contains one white chromosome, one black (Figure 51, F 1). This will happen in every pair of the two races that mate together; every family will have a white-black pair of chromosomes.

In ordinary fission, however, the black and white do not separate, but each merely splits, so that all the individuals produced have this white-black chromosome pair (F 1). This is also just the situation of affairs in the first generation of offspring (the "F 1 generation") from a cross in higher organisms; this $\mathbf{F} 1$ generation is composed of individuals that are alike with respect to their characters.

But suppose that after a long series of generations, the white-black individuals mate among themselves (as at P 2, Figure 51). What will happen?

In all the individuals, one of the two chromosomes of this pair will be gotten rid of at the second maturation division, leaving the other. In half the cases it will be the black chromosome that is left; in half the white one. That is, half the individuals will have black chromosomes in their migratory and stationary nuclei, while half will have white ones.

Of those that contain black chromosomes, half will mate with other individuals that contain black ones, half with those that contain white ones. Similarly, of course, of those 


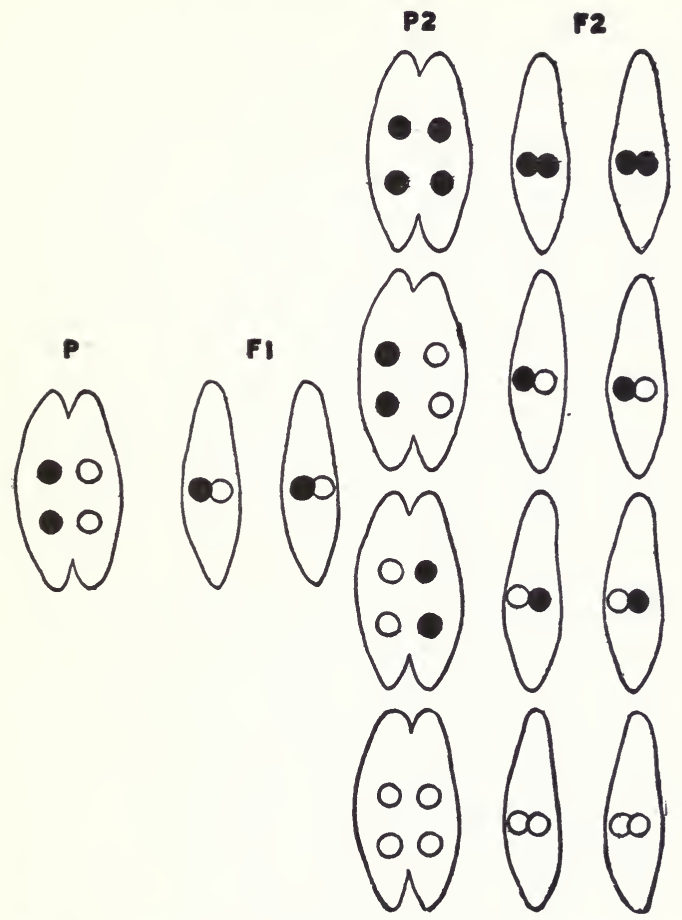

Figure 51. Diagram to illustrate how Mendelian inheritance would occur in an infusorian (Paramecium). The circles represent a pair of diverse chromosomes, the diversity being indicated by making one black, the other white. The diagram shows that in successive conjugations these would be distributed according to Mendelian rules. After the first conjugation (P), the ex-conjugants and their descendants by fission $\left(\mathrm{F}_{1}\right)$ would all have one black, one white, chromosome of this pair. At the next conjugation $\left(\mathrm{P}_{2}\right)$, by the varled reductions and matings the ex-conjugants and their descendants by fission $\left(\mathrm{F}_{2}\right)$ would exist in the proportions:-1 white-white: 2 white-black: 1 black-black. 
that bear white chromosomes, half will have mates with black chromosomes, half with white. Then all the different combinations so producible are shown at $\mathrm{P} 2$, Figure 51; each of these combinations occurs as frequently as any other. By two of the four combinations we get offspring (ex-conjugants, F 2) with one chromosome white, one black; by one we get offspring with both chromosomes white; by one, offspring with both chromosomes black. Summing up the eight offspring, we get the following proportions for the offspring of the generation F 2:

2 white-white +4 white-black +2 black-black.

Now this is exactly what is called Mendelian inheritance. If we call white $A$ and black $a$, the proportions give the familiar Mendelian formula

$$
\mathbf{A A}+2 \mathbf{A a}+\mathbf{a a}
$$

Any other chromosome pair, or any character that depends on a chromosome pair, will give the same result.

We have found then, so far as knowledge has gone in this direction, that mating produces the same kinds of results in the Protozoa that it does in higher animals; it gives biparental inheritance, and also gives many diverse hereditary stocks, and these results are produced in the same way in the Protozoa that they are in higher organisms.

\section{The Effect of Mating on the Stock}

What effect has this on the entire species in which mating occurs? To answer this question, another fact as to conjugation is of importance.

Assortative Mating:-When we place together in the same vessel members of two different races of Paramecium, one having large individuals, the other small ones, and then induce conjugation, we observe a surprising fact. Members 
of each race mate only with members of their own race; the large individuals only with other large ones; the small individuals only with other small ones (Figure 52). There is no crossing between the two races when they thus differ considerably in size. This is a highly inconvenient fact when one wishes to study heredity in such crosses!

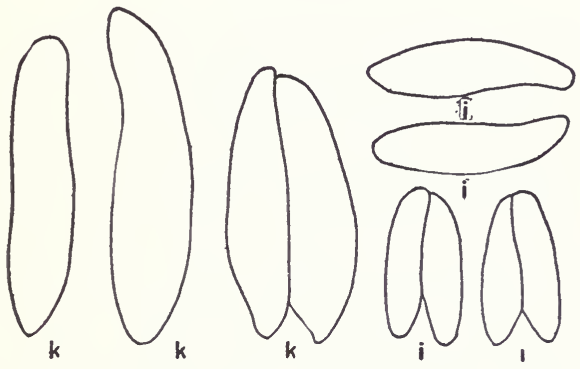

Figure 52. Conjugants and non-conjugants from a culture composed of a mixture of two races ( $k$ and $i$ ) of different size, of Paramecium aurelia. The members of the race $k$ are larger than those of the race $i$; only members of the same race mate together. After Jennings, 1911 .

The same thing is observed when a culture of Paramecium contains individuals of many different sizes (whether of the same race, or of different races). There is a marked assortative mating; that is, individuals of the same size tend to mate, while individuals of diverse size do not readily mate (Figure 53). There are some exceptions; we find a few instanees in which a small individual has mated with a larger one, but such cases are rare. In general, we find that all the pairs can be arranged in a rather regular series such as Figure 53 shows, - the two members being of about the same size.

This assortative mating takes place with respect to other 
characters also. It has been demonstrated by careful study that it occurs in Paramecium with reference to rate of fission; the two animals which mate have on the whole similar rates of fission. It appears clear that the mating is between animals of similar physiological characteristics.

Such assortative mating has been shown to occur with respect to size in certain other infusoria,-noticeably, Blepharisma (Watters, 1912) and Anoplophrya (Collin, 1909). Assortative mating is common, too, in higher animals and man. It is well known how strong a reluctance

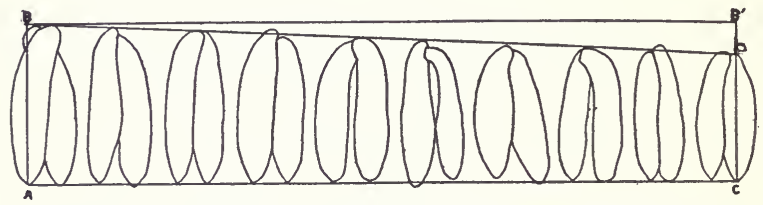

Figure 53. Pairs from a single race of Paramecium aurelia, illustrating assortative mating; individuals alike in size mate together. The lines A-C and B-B ${ }^{1}$ are parallel. After Jennings, 1911.

there is in man for strikingly different races to mate; a reluctance that is reenforced by all sorts of social and legal regulations (which regulations, of course, are manifestations of the biological characteristics of the organisms). In the case of blacks and whites among human beings, for example, an observer from Mars, examining in the United States the two stocks objectively, would find that in the overwhelming majority of cases white is mated with white, black with black,-although some exceptions occur.

In higher animals this assortative mating manifests itself in details also, as it does in Paramecium; study shows, for example, that on the whole tall persons tend to mate with tall, short with short. Although detailed studies have been 
made for but few cases, this tendency for like to mate with like, and to refuse to mate with unlike, probably exists in considerable degree throughout the world of organisms. This is one of the important facts to be reckoned with in attempting to get any general picture of the results of mating.

General Results of Mating:-To a picture of the general results of mating we now turn. In what respect does the world of organisms, or any particular group of organisms, differ from the condition which we would find if no mating occurred? We leave out of account here the results of the replacement of the old active nucleus by the reserve nucleus, since this is not a distinctive feature of mating, occurring as it does in the infusoria without mating; and in most organisms not occurring even at mating.

We hear it maintained on the one hand that mating produces variation; some assert, indeed, that it is the great source of variation. On the other hand, some maintain that the result of mating is to prevent or destroy variation; to keep the species of organisms uniform. Facts can be adduced that support both these propositions.

The difficulty here is that the expressions "increase variation" or "decrease variation" are ambiguous, and that neither of them precisely touches the essential point. The increase and decrease of variation are mere diverse aspects of what really occurs; sometimes one of these may result from mating, sometimes the other. The really fundamental thing that mating does is to produce new combinations of hereditary characters. And in so doing it quite changes the face of the world of organisms.

We may illustrate this most simply by noticing again what happens in the case of the primary hereditary characters, the chromosomal packets; we know that the secondary char- 
acters follow the primary ones. If there are two races of organisms, in each of which both members of each pair of chromosomes are alike (so-called pure lines, or pure homozygotes), we may call their two sets of chromosomes
A B C D
a b c d
$A B C D$ and $a b c d$

Now, when the two sets are reduced in number by division into two groups, with one member of each pair in each group, evidently the only possible groups are A B CD from one race, and $\mathrm{a} b \mathrm{~cd}$ from the other; all the half nuclei of each race will be alike. When two half nuclei from the different races mate, all the resulting nuclei will show the combination
A B C D
$a$ b c d

That is, by the crossing of these two diverse races, progeny are produced that are all alike. A new combination has been produced,- - but only one combination from the original two. So the progeny of the cross will be uniform, while the parents were diverse. Variation has been greatly decreased.

But now suppose that the progeny, showing this. new and uniform combination, mate among themselves. We have already for another purpose examined the results of this (page 186); we found that a great number of different hereditary combinations would be produced, such as

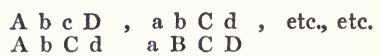

Progeny of 81 different kinds of hereditary combinations will result. By this mating of two parents that were just alike variation has been greatly increased.

Suppose that we compare this group of 81 diverse combinations with the two sets of grandparents. Has variation 
been increased or decreased? In the original stocks the two sets of parents were diverse in all their characters, while in their grandchildren, although there are 81 sets instead of two, they show all possible intermixtures and gradations. It may be maintained therefore that the result of mating has been to reduce the degree of diversity. And if we find the "coefficient of variation" for the two sets, which is a measure of how much on the whole the individuals differ from the average intermediate condition, we shall doubtless find this to be much greater for the two original stocks, where none of the individuals are like the average of the two sets, than for their grandchildren. By this measure, therefore, variation will be found reduced by mating. Walton (1915) has shown that precisely this is what occurs when diverse stocks of certain higher organisms are mated; from which he argues that mating decreases variation. The whole is an excellent illustration of the way in which average measures (like the coefficient of variation) may conceal important biological facts. What has happened is the production of many diverse hereditary combinations bridging the gap between the two that first existed. This is an increase of variation, if one means thereby an increase in the number of hereditarily diverse stocks; it is a decrease of variation, if one means by -ariation the average diversity from the intermediate condition. But this superficial increase or decrease of variation is merely a consequence of the underlying fact,- - the productions of new combinations through mating. ${ }^{4}$

4 Mast (1917) studied this phenomenon of increase or decrease in variability of the fission rate as a result of mating in the infusorian Didinium. He was not able to detect with certainty any consistent increase or decrease in the coefficient of variation as a result of mating. This need not surprise us, in view of the points brought out above; but Mast's data were in any case hardly such as to give dependable results on the matter. The study of variation was made on the results of experiments designed for entirely different purposes, so that the numbers of cases were too small to give significant data. Most of his coeff- 
The facts of assortative mating, of course, limit the extent to which new combinations of the characters are producible through mating. After stocks have reached a certain degree of diversity, assortative mating prevents their union, and so prevents the formation of stocks combining their characteristics. But this still leaves a wide field for the formation through mating of varied combinations of the hereditary characteristics of differing stocks.

We now come to one of the most striking biological results of mating. In this formation of new combinations, characters which were previously in diverse stocks become united in one individual. Sometimes the characters so combined are more or less incompatible; they do not develop well together; or they do not function harmoniously; or they produce secondary characters which do not function well under the particular conditions in which the organisms are found. Individuals resulting from such combinations may be weak or pathological; they may not develop at all; or if they do, they lack vigor, and do not multiply. Many examples of such consequences we saw in our study of the results of mating in Paramecium; many individuals or families produced died out, or were weak, multiplying slowly; or showed hereditary deformities. Other individuals on the other hand received combinations of characters

cients of variation were worked out on the basis of but 2,3 or 4 diverse lines of descent! In only one single case were there as many as eight lines in both the sets compared (conjugants and non-conjugants); in this case the variation was much greater in the lines that had conjugated. But it is obvious that such small numbers cannot give clear and consistent results, particularly when we find that the coefficients of variation brought to light range from 0 to 55.3 per cent. If probable errors had been worked out, they would doubtless have shown the comparisons of coefficients to be without significance. A study of the question whether mating actually produces lines with diverse hereditary characters would be of great interest in Didinium, as in any other Protozoa, but the determination of coefficients of variation is a most imperfect and uncertain index of this matter. 
that worked well together and under the conditions of the environment; the families produced were vigorous, some of them flourishing even better than the original stocks through whose mating they were produced. In the same manner a poor family of human beings may produce a Lincoln among its children; a combination of characteristics is formed by mating that never before existed and that meets the conditions of existence in a more vigorous and successful manner than the individuals from which it was derived.

Experimentally, or under natural conditions, of course these newly formed stocks in which life and reproduction are vigorous, gradually replace the weakened stocks, in which mating has resulted, not in reinvigoration but in degeneration; in the formation of combinations of primary hereditary characters that cannot develop vigorously under the conditions. The formation of such "degenerated" stocks is as much a characteristic of mating as the formation of more vigorous ones.

By this formation of new combinations with gradual replacement of the unenduring ones by those that are vigorous, the stocks in existence come to be very diverse from those that existed at an earlier time. Mating is a continued process of forming new combinations of the primary hereditary characters; of the chemicals on which the vigor and the nature of development depend; with suppression of the combinations that are weak or imperfect, leaving the more harmonious and vigorous combinations in existence, to carry the process further. 


\section{VIII}

Comparison of the Genetic Phenomena in the Protozoa with Those in Higher Organisms. General View of Development, Mating and Evolution.

YW far does the condition of affairs which we have set 1 forth for the lower organisms hold for the higher ones?

As we have remarked at various points in our earlier lectures, many, perhaps most, of the general relations are similar in the lower and higher organisms. But there are certain points in regard to which questions may be raised; particularly as to the origin of new hereditary characters. The points needing examination are mainly (1) as to the effect of the environment in causing inherited changes, and (2) as to the nature and extent of the changes in hereditary characters that arise in nature; with the relation of these to the process of evolution.

We have dealt in our fourth lecture with alteration of the hereditary constitution of lower organisms by external conditions. In the higher, more complex organisms the underlying conditions as to this are somewhat different from those found in organisms composed of but a single cell; though perhaps not so completely diverse as is sometimes conceived. But in the higher organisms there is a great mass of cells, the body, which finally disintegrates completely, without propagating itself by division; the body of the next generation is formed, not from the body of the preceding genera- 
tion, but by the divisions of a single cell, which is formed by the union of two half cells, one from each parent. This was illustrated in Figure 5, on page 21.

It is clear that if any of the later (secondary) hereditary characters are to become modified, this must be accomplished by some modification of the primary hereditary characters, -those passed on bodily from parent to offspring. For if two germ cells are exactly alike, the characters inherited through them are bound to be alike; two individuals that are to differ in their later hereditary characters must be diverse in their primary hereditary characters. If environmental agencies are to produce diversities that are to be hereditary, they must change the germ cells, in the particular way required to bring about the observed later changes in the body; for the body is derived from these germ cells.

This has always been the theoretical difficulty with the "inheritance of acquired characters,"-if we mean by that abused expression the inheritance of modifications produced directly on the body by the outer world. If the form of the hand is changed by certain outward conditions, how is that change to modify the primary hereditary characters in the germ cells, which are not directly touched by the given outer conditions, -in just such a way as to cause them to produce the same new form of hand in the next generation?

Theoretical difficulties of this sort of course must not be allowed to stand in the way of our recognizing how nature actually does operate, if she does indeed operate without regard to these difficulties. There is nothing so little worthy of confidence in science as assertions that particular events, not yet observed, are impossible; such propositions have been falsified a thousand times, and the careful man of science, will not permit his researches to be guided by them. But in this case the great weight of evidence thus far is that this 
particular theoretical difficulty corresponds to an actual one; that the direct effect of the environment on the body cells is not inherited through the germ cells in the next generation. It is only fair to say however that there is certain evidence produced by the Austrian investigator Kammerer (1913) in long-continued experimental studies on amphibians which seems to imply such inheritance through the germ cells of changes primarily produced in the body cells of the animal. But practically all students of biology will agree that this evidence is far from establishing heredity of this sort, and that the overwhelming mass of evidence is against it.

But it is another question whether external agents may not act directly on the germ cells, in such a way as to induce them to produce a body with new characteristics, and to transmit the same changes by cell division to the germ cells that are to produce the later generations, so that these too show the altered hereditary characters. This sort of action would correspond to the hereditary changes produced by external agents in the Protozoa and bacteria, such as we described in Lecture 4.

There is no theoretical difficulty whatever as to this; the difficulties are purely observational ones; it turns out that such changes do not occur so readily or frequently as one would expect. There is no a priori reason why the substances of the germ cells should not be as readily altered as any other mass of chemicals. But in practice it turns out that most agents which produce chemical alterations of the germ cells at the same time kill the organism. Further, the germ cells, like other living systems, have a great tendency to compensate for any disturbances induced them; their condition is one of stable equilibrium, in which an alteration is followed by a return to the original condition. Add 
to this the fact that in higher organisms the germinal material is commonly hidden deeply within a great mass of body cells, by which its surroundings are kept uniform and it is protected from marked changes of all sorts, - and it becomes more intelligible why in higher organisms inherited changes due to the action of the environment are much less commonly observed than general theory might lead us to expect. In the bacteria and Protozoa the germinal material is not protected by a great mass of body cells, but is more directly exposed to the action of environmental agents, so that in these, heritable results of environmental action are better known.

When we examine the experimental evidence on this matter in higher organisms, we find that scientific opinion looks upon it as being in a somewhat less satisfactory condition than appeared to be the case a few years ago. Cases had been described in which the inheritance of the action of the environment on the germ cells appeared clear; and the evidence on these particular cases has not altered. But the lack of further confirmation, of other instances; the failure of other tests under similar conditions, has shaken the opinion of most students of biology as to the conclusiveness of the evidence that had been given, and has made them inclined to wait for further evidence before accepting the principles of action involved. No subject in biology is more in need of further work than this one.

The principal evidence for actual modification of the germ cells by the environment in such a way as to cause the appearance in the body of new hereditary characters has come perhaps from the work of Standfuss (1906) and Fischer (1907); of Tower (1906); of Kammerer (1913), and of Stockard (1913); these at least are typical. It is not our present task to give an account of these researches, 
but a few words of comment will aid in obtaining an outline of the present situation in science.

Standfuss and Fischer, by the application of low temperatures to the pupæ of certain butterflies, induced in a small proportion of the adults the appearance of darker tinges of color than usual. In a small proportion of these aberrant adults, the modified color reappeared in later generations; it was hereditary.

Tower tried the effects of hot, moist conditions on the potato beetle at the time that the germ cells were undergoing their growth and transformation. He found that the germ cells so treated produced a considerable proportion of individuals differing from the typical ones; lighter individuals and darker ones. And in later generations these aberrant colors showed themselves to be hereditary.

Kammerer experimented for many years in breeding various sorts of amphibians, attempting through climatic changes, alterations of temperature and moisture, changes in color of the background on which they live, and by other means, including operations, to modify their colors, habits and other characters. According to his detailed reports, published in the technical scientific journals, he has been remarkably successful in this; many sorts of diversities in environmental conditions have produced inherited alterations; including even operative procedures. These positive results have come to him so easily and regularly, in experiments of a sort in which others have practically universally reached negative results, as to arouse in most investigators a feeling that they must be confirmed by others before they can be accepted. It must be said however that Kammerer's work appears to have been done with great thoroughness and care, and he has given full account of his methods and results, in such a way as to leave little room for criticism of details. On the 
other hand the phenomena with which he deals are excessively complicated and variable, making errors of interpretation easy to one who is strongly convinced of a particular doctrine, as Kammerer evidently is of the inheritance of environmental effects. Some results and interpretations reported by him have put a strong strain on the powers of acceptance of other investigators; notably his report of the inherited effect of cutting off a certain organ in the ascidian; ${ }^{1}$ and his report that when the ovary of a given sort of salamander is transplanted to the body of another kind, the germ cells of this ovary transmit the characters of the body to which they were transplanted. Kammerer's work is distinctly in need of confirmation.

Stockard dealt with the effects of alcohol on the germ cells, and through these on the later generations of offspring in the Guinea pig. He found that continued administration of alcohol to the parents so injures their germ cells that their progeny and their descendants of later generations are weak, imperfect, diseased, deformed, in many ways.

Somewhat similar results had been reached years before by Brown-Séquard (1869), in studying the results of mutilation of the parent in Guinea pigs. Repetition of his work by later investigators has not convinced students of the subject that it was correctly interpreted; it is believed that he was dealing with diseased stocks. The study made by Pearl (1917 $a$ ) of the effects of alcohol in the fowl did not bring to light any such results as Stockard's on the Guinea pig, so that there is on the whole a tendency to suspend judgment as to the interpretation of the results until Stockard's work has been repeated on a new stock.

Thus all along the line there is a feeling of uncertainty

${ }^{1}$ See the comment of Castlé, 1916a, page 29, on this point. 
and a desire for further tests before judgment is passed as to the inherited effects of environmental action on the germ cells, in higher organisms; more work along these lines is greatly needed.

But the results set forth are, in all cases save perhaps certain of those described by Kammerer, of the sort that agree in principle with the inherited effects of environmental action in the lower organisms, as described in Lecture 4. The action of the external agents was directly on the germ cells, modifying the primary (directly transmitted) hereditary characters. In consequence the later or secondary characters were altered. Such cases are perhaps better established in the Protozoa and bacteria than in higher organisms.

As to the nature and extent of the changes in hereditary characters arising in higher organisms, aside from the direct effects of environmental action, an enormous volume of work has been done. In higher organisms it is a prevalent conviction that changes in the hereditary characters occur by mutation. What relation has this and the facts on which it is based to the hereditary changes seen in the Protozoa,in such a case as Difflugia, for example?

The concept of mutation has been based, in different minds, on a number of different points,-sometimes held separately, sometimes together. One basis for the distinction of mutations from other variations is this: Many variations in the characteristics of organisms are not inherited; such are the common superficial effects of environmental diversities. We have given illustrations of this in our account of the Protozoa. It is therefore convenient to have a distinctive name for those that are inherited, and some call these mutations. In this usage, of course it is a mere matter of definition to state that any new heritable 
variation is a mutation. The word so used implies nothing as to extent or nature of the variations that are herit. able; it is a name for all that occur.

But in many minds the term mutation means more than this. Many new heritable characters in higher organisms are found to be, when the character has reached its complete development in the adult, changes of considerable extent. They differ from the original condition by a large step; they affect many parts of the organism; or profoundly change particular organs. Such were most of the hereditary changes found by de Vries (1901) in the evening primroses, Enothera; from this work arises the general use of the word mutation for hereditary changes. Such too are many of the hereditary changes observed in the fruit-fly, Drosophila, by Morgan and his associates. Thus, in the typical individuals the eyes are red; these sometimes produce offspring in which the eyes are white, and this mutation is inherited. The hereditary change has come, not by minute changes of shade, gradually altering from generation to generation; but by a complete change in one generation from red to white.

From such cases, the word mutation has come to mean in the minds of many persons an extensive change; a sudden jump from one condition to another; a "saltation." And the statement that evolutionary changes occur by mutation has come to mean that they do not take place in gradations; in minute, almost imperceptible alterations from generation to generation, but always by large leaps. Possibly this is the usual idea of what is meant by the mutation theory of evolution.

Such slight changes as we have described in the preceding lectures as occurring in Difflugia and other Protozoa do not agree with this idea. Is there a contrast in this respect between what occurs in the higher and the lower organisms? 
It is natural that an alteration of a primary hereditary character in the germ cell of a higher organism should, when the long development from that germ cell is completed, produce a much more extensive and more marked effect than in a Protozoan. For in the latter it is the same cell that is altered which forms the adult, with relatively little development, and with no intervening multiplication of cells. But in the higher organism the altered germ cell goes through a great number of cell divisions, accompanied by continuous interactions of the different substances in the nuclei, resulting in an enormous increase in differentiation, in numbers of cells, and in bulk. All these cells, and this entire bulk, may therefore show the results of the slight original change. If some substance necessary for the production of the red eye color of the fruit-fly were omitted from the germ cell, it is probable that the change in the germ cell would itself be so slight that it could not be detected by any physical or chemical tests at present available. It is little more than changes corresponding to this that we may expect to find in the organisms made up of but one cell.

But must a hereditary change in the adult characters of a higher organism necessarily be such a saltation; a change of large extent? On this point the state of knowledge has greatly changed with the thorough studies made in recent years, although the change has as yet been little appreciated outside the field of specialists working on these matters. We shall attempt to give a brief sketch of the position of this question in higher organisms, for comparison with what we have seen in the lower ones.

It has been found that in many higher organisms it is possible through long continued breeding with careful selection of the parents, to gradually cause a change in the hereditary characteristics shown by the stock. Often this change 
is a mere quantitative alteration, in the extent or intensity of pigmentation or other characteristics. In many cases such alterations have occurred in characters that in other respects behave like "single unit characters." Such work was done on the rat by Castle and his associates; on Drosophila by MacDowell, Zeleny and Mattoon, Reeves, Morgan, Sturtevant, and others.

Two views have been held by investigators as to the nature of the change in such cases. Castle and a number of others have long held that there was occurring a gradual-change, perhaps merely quantitative in nature, in the single unit factor on which the adult character depends. On the other side, many have maintained that these gradual alterations are due to the fact that the adult character depends on many distinct genes or unit factors, each affecting the adult character but little. By selective breeding many of these factors are gradually accumulated in one set of progeny, few in the other; so that the adult features become slowly very diverse. That is, it is maintained that the apparent changes in the hereditary characters are really due, like all Mendelian inheritance, to recombinations of the existing factors.

This explanation, commonly called the hypothesis of multiple modifying factors, has recently been accepted, on the basis of crucial experiments, by Castle himself. ${ }^{2}$ There can hardly be doubt that it is correct for at least most cases of this kind.

Let us, therefore, accept this explanation, and proceed to an examination of its relation to the questions in which we are interested. What bearing have the facts, so interpreted, on the nature of hereditary variations and on the method of evolution?

${ }^{2}$ Castle, W. E., Proc. Nat. Acad., April, 1919. 
In no other organism have heritable variations been studied so thoroughly as in Drosophila, and no other body of men have been more thoroughgoing upholders of mutationism and of the multiple factor explanation of the effects of selection, than the students of Drosophila-Morgan, Sturtevant, Bridges, Dexter, Muller, MacDowell, and the others. We may therefore turn to the evidence from Drosophila with confidence that it will be presented with fairness to the mutationist point of view. We shall first ask (1) what we learn from the work on Drosophila as to the possibility of finding finely graded variations in a.single unit character. Next we shall inquire (2) as to the relation of the assumed modifying factors to changes in hereditary constitution; to the nature of the effects of selection.

1. First, then, what are the facts as to numerous finely graded variations in a single unit factor? Here we have certain remarkable data as to the eye-color of Drosophila; data that are of great interest with relation to the nature of evolutionary change. This fruit-fly has normally a red eye. Some years ago a variation occurred by which the eye lost its color, becoming white, a typical mutation. Somewhat later another variation came, by which the eye color became eosin. By these wonderfully ingenious methods which the advanced state of knowledge of the genetics of Drosophila has made possible, it was determined that the mutations white and eosin are due to changes in a particular part of a particular chromosome, namely, of the so-called $\mathrm{X}$-chromosome, or chromosome I. And further, it was discovered that the two colors are due to different conditions of the same locus of the chromosome; in other words, they represent two different variations of the same unit. Moreover, the normal red color represents a third condition of that same unit.

Somewhat later a fourth condition of this same unit was 
found, giving a color which lies nearer the red, between the red and eosin; this new color was called cherry. So we have four grades or conditions of this single unit character.

And now, with the minute attention paid to the distinction of these grades of eye color, new grades began to come fast. In the number of Genetics for November, 1916, Hyde adds two new grades, one called "blood," near the extreme red end of the series, the other called "tinged," near the extreme white end; in fact, from the descriptions it requires careful examination to distinguish these two from red and white, respectively. Thus we have now six grades of this unit. And in the same number of the same journal, Safir (1916) adds another intermediate grade, lying between "tinged" and eosin; this he calls "buff." All these seven grades are diverse conditions of the single unit factor, having its locus in a certain definite spot in the $\mathrm{X}$-chromosome. Such diverse conditions of a single factor are known as multiple alleiomorphs.

So, up to date we know from the mutationists' own studies of Drosophila that a single unit factor presents seven gradations of color between white and red, each gradation heritable in the usual Mendelian manner. These grades are the following: (1) red; (2) blood; (3) cherry; (4) eosin; (5) buff; (6) tinged; (7) white.

It would not require a bold prophet to predict that as the years pass we shall come to know more of these gradations, till all detectible differences of shade have been distinguished, and each shown to be inherited as a Mendelian unit. Considering that the work on Drosophila has been going on only about seven or eight years, this is remarkable progress toward a demonstration that a single unit factor can present as many grades as can be distinguished, that the grades may give a pragmatically continuous series.

Besides showing that a unit factor may thus exist in numerous minutely differing grades, this case shows that a 
heritable variation may occur so small as to be barely detectible. Although the variations do not usually occur in this way, the case present the conditions which would allow of a gradual transition from one extreme to the other, by means of numerous intermediate conditions.

2. But, as we have seen, the gradual changes in hereditary characters seen in selective breeding usually do not occur in this way, but rather by the slow accumulation of many factors each having a slight effect,--the multiple modifying factors. But what sort of things are these factors and what is their relation to actual changes in the heritable constitution of the organism?

Our direct experimental knowledge of these "modifying factors" is scanty; it comes mainly from the studies of Drosophila. We find data as to certain known modifying factors by Bridges (1916) in his important paper on nondisjunction of the chromosomes. And here we are taken back again to the series of eye colors, and indeed to one particular member of the series, the middle member, called eosin. Bridges tells us that he found a factor whose only effect was to lighten the eosin color in a fly with eosin eyes; this factor indeed nearly or quite turns the eosin eye white. This factor Bridges calls "whiting." Another factor has the effect of lightening the eosin color a little less, giving a sort of cream color; this is called "cream b." A third factor dilutes the eosin color not so much; it is called "cream a." In addition to these, Bridges tells us that he has discovered three other diluters of the eosin color; we will call them the fourth, fifth, and sixth diluters. And finally Bridges tells us of another factor whose only effect is to modify eosin in the direction of a darker color; this factor he calls "dark." None of these factors has any effect save on eosin-eyed flies.

As you see, these things add tremendously to our grada- 
tions in eye color. We had already been furnished seven grades, from white to red; now we have seven secondary grades within a single one of these seven primary grades. Our list of gradations of eye color in Drosophila therefore takes now the following form:

Heritable grades of eye color, due to diverse variations of a single unit located in Chromosome I.

1. White

2. Tinged

3. Buff

4. Eosin.

5. Cherry

6. Blood

7. Red
Variations that give modifications of the intensity of eosin, but are located in other chromosomes.

Here again then we have minutely differing conditions of a single shade of color, brought about by seven modifying factors.

But what are these modifying factors? And here we come to the essential point. These modifying factors are themselves alterations in the hereditary constitution. Bridges leaves no doubt upon this point. He lists and describes them specifically as mutations; as actual changes in the hereditary material.

What then is the difference in principle between such cases and the theory of gradual alterations in a single unit factor? The difference is that in the case of the multiple modifying factors the minute changes occur, not all in one factor -in one locus of the chromosome- but in a number of diverse parts of the germinal material; this appears to have been clearly demonstrated. But this is a matter of detail; it does not touch the fundamental question.

This fundamental question is as to the occurrence of these minute changes in the hereditary constitution, and as to the 
possibility of getting therefrom by selection various grades of a given external characteristic. In this, so far as I can see, there is complete agreement.

It appears then that under the recent careful studies made, it can no longer be maintained that hereditary changes, even in higher organisms, must be large leaps or saltations. They may be of this character, but they may equally well be graded changes so slight as to be hardly detectible when taken singly.

This appears to be recognized by those who have proposed and defended the mutation theory. De Vries (1916) in a recent summary of the theory emphasizes throughout extensive mutations, and speaks repeatedly of their origin as "sudden and without transitional conditions," but admits also that "not only very small, but also much greater" differences between species arise all of a sudden ("mit einem Sprunge"); and that most mutations affect only a single character. He sets forth further that in a single stock one such mutation after another may arise, at intervals, until in the course of time the stock has become very diverse from the original one; and has become differentiated into a number of different types on which selection may act. Now, so far as the mutations are "very small," the condition after but one or a few mutations had appeared would be practically indistinguishable from a "transitional condition" to the state after many mutations had occurred. Morgan (1917) recently insists that it must be recognized and has always been urged by de Vries that "mutations may be very small so far as the character change is concerned."

The true and important points insisted on by the mutation theory appear to be these:-

(1) There are many differences ("variations") between individuals that are not heritable. Hence by selection of such diversities no evolutionary change is produced. 
(2) Actual hereditary changes in characters occur rather rarely. This is apparently what is meant by the statement that they appear suddenly ("sprungweise"); for a time they do not exist; then they do. (But the changes thus suddenly occurring may be so minute as to be hardly detectible until later changes in the same direction have accentuated them.)

(3) Heritable changes may and often do occur in large steps, so far as their effect on the developed characters of adults is concerned. (This is an important fact; equally important is the fact that heritable changes may be, and often are, very minute.)

(4) Appearances indicate that the changes are analogous to (or actually are) chemical changes. When one chemical compound changes into another, there is, it is held, no transitional condition between the two, and the same is believed to be true for hereditary variations. This conception of the nature of hereditary variations accounts for the fact that they often show in the adult as changes of large extent; and at the same time it fits equally well the minutely graded hereditary changes that likewise occur. For there is no change so minute that it may not be chemical in its nature. In the immense organic molecule, with its thousands of atoms, a shift of a single radical or single atom from one position to another is a chemical change, though it may make a difference so slight as to be almost beyond detection by the most refined means.

Whether a doctrine embodying these ideas differs from that set forth by Darwin to such an extent as to deserve the name of a new theory may be doubted. This will be a matter of individual opinion.

The doctrine that hereditary changes must occur by large steps evidently cannot be held. But what bearing on the method of progressive evolution has the fact that they often do occur by such steps,- not by a series of gradual altera- 
tions? In the eye of Drosophila variation may occur from red to white directly, without transitional stages; or from any grade to any other; the continuous scale of colors we have mentioned is obtained only by arranging the steps in order. Some maintain therefore that evolution has occurred by such large steps, not by gradations. This conception has evidently lost the compelling force it seemed to have before hereditary variations in minute grades were detected. The very facts in such an organism as Drosophila show that there is nothing to prevent a passage from one extreme to another by minute changes, such as are held to occur by palæontologists and selectionists. Further, in such cases as the eye-color of Drosophila we are dealing with characters that are already highly developed, and the changes we observe are mainly retrogressive. We know, for example, that the red eye color of Drosophila is formed by the cooperation of many separate parts of diverse chromosomes; it is a highly complex product of evolution. Now, we find that one or another of these parts may suddenly cease to perform its function, so that the red color is not completely formed; there is a sudden change in it; or it may disappear completely. But it may be doubted whether this implies that in the original production of this complex character, with its numerous underlying functional parts, there was the same change by sudden large steps. Is.there any reason to suppose for example that at one time there was a complete eye save for the absence of the red color; and that this suddenly appeared? Our knowledge that this red color is made by the cooperation of many diverse parts makes such a notion almost inconceivable. Destructive changes in a fully formed character, such as we see in the large majority of cases of mutation, could hardly be expected to throw light on how that character was built up. The observed 
facts leave readily open the possibility of the building up of a character by minute graded changes.

In essentials, therefore, the study of mutations, when carried so far as in Drosophila, is not in disagreement with our observations of gradual variation in the Protozoa nor with the conclusions of palæontologists as to the gradual development of the characteristics of organisms in past ages.

These conclusions of the palæonologists are well stated in the recent work of Osborn (1917). He sets forth that in following given stocks from earlier to later ages, characters arise from minutest beginnings and pass by continuous gradations to a highly developed condition. This seems in agreement with the experimental results on both higher and lower organisms, as I have tried to set them forth. The palæontogolical evidence, he holds further, indicates that the hereditary changes as one passes from age to age do not occur in random directions, but follow a definite course, which might seem to have been predetermined in the constitution of the organisms, or otherwise. In the experimental work on the lower organisms little that indicates this has thus far been observed. By selection we can move in more than one direction; though it is also true, of course, that the variations possible are limited by the constitution of the organism. The experimental work has hardly gone far enough to offer important evidence on this problem.

There is one other point in the work on higher organisms that we may briefly consider. This is the point made by Bateson (1914) in his Presidential Address before the British Association, and further developed in a recent paper by Davenport (1916). It is the paradoxical proposition that since practically all observed variations are cases of loss and disintegration, we are driven to suppose that evolution has occurred by loss and disintegration. Davenport com- 
bines this idea with the theory that these disintegrating variations follow a definite course, predetermined in large measure by the constitution of the disintegrating material.

There are two points that need consideration in dealing with this theory. The first is one of observational fact; although it is true that many mutations appear to be cases of loss and disintegration, yet there is no indication that this is the case in such results of selection as have been described in the Protozoa; heritable variations are not limited to any particular direction.

But secondly, it appears to me that this conclusion that evolution is by disintegration and loss is based on an error in logic, which, being detected, puts it out of consideration. As we examine the series of organisms, from amœba to man; or as we examine the palæontological series, we find a gradation from those showing little visible differentiation to those showing great visible differentiation; the problem of evolution is as to how the passage was made from those visibly little differentiated to those visibly highly differentiated.

But now, according to the doctrine we are considering, when we come to examine the actual changes in hereditary characters, we detect on the whole only the disintegration of organisms already visibly differentiated; only a change from greater visible differentiation to less.

The doctrine then proceeds to draw from this fact the absurd conclusion that the visibly more differentiated must have arisen from the visibly less differentiated by decrease in the differentiation of the latter!

So preposterous a conclusion can be drawn only from the fact that while in our premises we are talking of visible increase and decrease of differentiation, in the conclusion as ordinarily drawn the ground is shifted to mean something entirely different, -an inner, invisible, purely theoretical 
kind of disintegration and differentiation. If we recall that we were dealing in the premises with visible increase and decrease of differentiation, and that therefore in the conclusion we must deal with the same, the absurdity of the conclusion becomes manifest. All that we can legitimately conclude, if we accept the premise that the observed changes in hereditary characters are cases of loss and disintegration, is that we have not seen the process of evolution occurring. But we are not compelled to accept that premise. In the lower organisms at least it cannot be asserted that all changes of hereditary characters are cases of loss and disintegration.

General View of the Processes and Problems of Development, Mating and Evolution, in the Light of What We

Find in Lower Organisms

Let us now attempt an outline of what our examination of the processes of mating and development have shown us in the lower organisms, in so far as it agrees with what we find also in the higher organisms.

We saw at the beginning that each species of organism, so far as studied, is differentiated into many slightly diverse stocks, each diversity hereditary. This we found to be true both in Protozoa and in higher organisms. We saw, too, that in simple reproduction from a single parent, by division of a cell or of an individual, there is a high degree of constancy in the hereditary characters of these slightly differing stocks. The constancy is so great that for a long time the search for hereditary changes was at a standstill; the stocks seemed permanent.

But with intensified study, it was found that in the Protozoa changes in the hereditary characters do occur even in reproduction by simple division. Such an organism as Difflugia gradually differentiates, even without mating, 
into slightly differing stocks, similar to those found in nature. The changes are either continuous, or by steps so slight that single ones are hardly detectible.

In the higher organisms, where the matter is greatly complicated by the fact that most reproduction is from two parents, it first appeared that gradual or minute hereditary changes did not occur. But as we have tried to bring out above, with more thorough study it has come to light that such changes do occur, as well as do hereditary alterations, which, when they reach the adult condition, form a sudden marked change. In this way all contrast in principle between what we find in the lower organisms and what we find in the higher ones disappears. In both the process of evolution by minute gradations is visible.

Then we proceeded to examine how far in lower organisms diversity of external conditions brings about hereditary changes. Here again the first examination, even though long continued, seemed to show constancy; diversity of external conditions appeared to have no permanent effect on the stocks. But again, intensified study reveals that the hereditary characters gradually do becone changed by diversities of external conditions. Through such diversities, continuing for great numbers of generations, single stocks, uniform in their hereditary characters, gradually differentiate into many with faintly differing hereditary features. Again the process is gradual, or by steps so small that single ones are imperceptible. In higher organisms the state of knowledge on this point appears less satisfactory. But the evidence so far as it goes indicates that the processes here are in agreement with those in lower organisms. Apparently diversities in external agents may, under conditions which seem rather rarely met, so modify the germ cells that they produce progeny with changed hereditary char- 
acters. On the other hand there is little indication that when an agent produces a direct effect on a part of the body, this so changes the germ cells that in later generations they produce bodies with the same alterations.

All together we find then, that even independently of any mating processes, diversity of stocks is being produced, but most slowly and gradually. -

Next we turned to a study of reproduction from two parents, and its relation to these general questions. We found that while in many organisms (particularly the higher ones), the two individuals or cells that mate are unlike, belonging to separate sexes, this seems not to be universal. Mating is apparently often between like parts. This appears clearest in the final act of mating, the conjugation of the chromosomes, for in these there is no indication of a sex difference. But it seems to be true also in many cases for the germ cells and for the individuals that mate. It is certainly not clear that sex diversity is a general and fundamental requisite for mating; rather does the contrary appear true.

Yet where sex diversity does occur, as in the higher organisms, it is manifest in the most fundamental features of the organism. Every cell of the male, in many organisms, differs from every cell of the female, and precisely in the most fundamental features of the cell; in the nuclei; and in the essential chemical operations in which the nuclei are involved. But this seems to be a condition derived from the simpler state where no such diversity exists, but in which mating nevertheless occurs.

In search for what is fundamental in mating and its results, we came upon theories that mating produces rejuvenescence; that mating is a necessity for continued existence and multiplication; that without it vitality is lost; 
and that it must take place in order that the lost vitality shall be restored. But when we examine the evidence on this in lower organisms, we find a whole series of facts that will not range themselves under this doctrine, along with some that will. After mating, some organisms are less vigorous than before; some little altered; some more vigorous. The latter may be held to show rejuvenescence. But the general result is to produce many diverse stocks with new sets of hereditary characters. Some of these new combinations show greater vigor, others less; and they differ in many other ways. Such diverse stocks resulting from mating show'similarities among themselves, resulting from their dependence on the union of two parents; that is, they show biparental inheritance. But the stocks produced by a given mating differ, too, in their hereditary characters.

These relations, as yet little known in the lower organisms, receive illustration on a vast and conspicuous scale in the higher organisms, where they are known as Mendelian inheritance. The offspring of a given pair are more alike than are the offspring of diverse pairs. Nevertheless, the offspring of a single pair show combinations of hereditary characters diverse from each other and from their parents.

Examining the minute processes that occur in reproduction from two parents, we find that there is a visible formation of new combinations of the chemicals on which development and function depend; new combinations. of the primary hereditary characters. These chemicals are in visible packets, and the method of forming new combinations of them can be seen under the microscope. Even when, as often happens, the mating is between parts of the same nucleus of the same cell, the processes are such as to bring about new combinations of the primary hereditary substances; organisms with new combinations of hereditary 
characters are produced. These recombinations occur in the same general way in the lower and the higher organisms.

This formation of new combinations of the primary hereditary substances is then the general feature of mating. It is this of which we were in search when we asked: Is there any general result of mating, comparable with the production of energy as the general result of the taking of food? Mating is a process of forming new combinations of the primary hereditary materials.

As a result of these new combinations, the organisms produced are very diverse in their hereditary characters. In the infusorian some are weak and unenduring; the things combined do not work together harmoniously; they die out. Others are strong and vigorous; they persist and multiply. In other organisms similar differences appear, along with diversities in respect to all possible hereditary characteristics. Thus mating steadily changes the face of organic nature, continuously producing new combinations, some of which are extinguished, while others flourish.

This process is greatly assisted in the lower organisms by the fact that after a set of new combinations is produced by mating, each combination is multiplied greatly by vegetative reproduction, which does not make a change in the grouping. Thus each combination is given an opportunity to meet many diverse external conditions, with some of which it may work in harmony; further, the number of possible diverse combinations which may result from the next period of mating is greatly increased.

This continued formation of new combinations is the great corrective of the uniformity which would result from more rigid laws of heredity. In general, no one can predict what combinations will actually result from a given mating, for the number possible is much greater than the number 
that can be realized. In other words, no one can predict with certainty the characteristics of the offspring to be produced by a given pair. Parents in which certain characteristics are developed in but a mediocre degree may produce by their union offspring in which these characteristics are developed in a high degree, as we saw in Paramecium that parents of low vigor may produce a few offspring of high vigor. This is as true for the qualities which in human individuals we tend to class as good or bad, as for vigor in Paramecium. From mediocre parents may arise, by the formation of new combinations of the hereditary material, offspring that are distinguished for good or for ill.

This is the fact that undermines all exclusively aristocratic theories of breeding and inheritance in such an organism as man; this is the possibility that must underlie any democratic theory of society and of progress. Possibly from the great mass of mediocre humanity there may arise by new combinations in every generation so great a number of distinguished men as to make the contribution of offspring from the relatively few distinguished individuals unimportant. It is not certain that the relative infertility of the intellectual classes decreases the existing proportion of intellectual men. It may be that there continually arises from the great average mass of mankind a proportion of distinguished men that remains relatively constant, even though these distinguished men may not reproduce at all.

To return to our general relations, this process of recombination does not evidently, of itself, result in the production of any new characteristics. We cannot say positively that it does not, but if the chemicals on which development depends - the primary hereditary characters-were permanent, unchangeable things, then recombination could produce merely kaleidoscopic regrouping of these; and the 
number of possible diverse combinations that could appear would be definitely limited. All change would be a regrouping of what already exists.

Therefore it becomes most important to examine what happens when there is no such regrouping, in heredity from a single parent. Here we discover, as we have already set forth, that actual changes in the hereditary materials are occurring, independently of recombinations. New characteristics appear that are heritable.

Are such new characters bound up with the other primary hereditary characters, and subject to the same processes of recombination at mating? In Drosophila this question is answered clearly in the affirmative; the new characters that appear recombine at mating as do the old ones. There is little reason to doubt that they do so in all organisms.

Thus a new character arising in a particular individual having a given combination of characters, is transferred by mating to other individuals with other combinations. With some it may work harmoniously; with others, not. Further, several new characters arising in diverse individuals may by mating become transferred to a single one, where they may form a combination working more harmoniously together and with the environment than any that has before existed. Thus all sorts of combinations arise, of new and old characters, such as could not possibly occur without mating. Some are more vigorous and harmonious; some less; some fit one set of outward conditions; some another. Mating thus contributes enormously to the differentiation of organisms into hereditarily diverse stocks. Its general result is to give all sorts of combinations of characters, new or old; some persisting, others not. Added to the slow production of new characters, it greatly hastens the changes in organic nature that we call evolution. 


\section{WORKS REFERRED TO IN THE TEXT.}

(This is not a general bibliography of the subject, but only a list of the works to which reference is made. Where there is general reference to a large series of investigations by a particular author, as a rule only one reference is given, to some important contribution; from which the reader can follow up the subject if he desires. This is done particularly when the subject lies to one side of our main field.)

Ackert, J. E., 1916. On the effect of selection in Paramecium. Genetics, 1:387-405.-Agar, W. E., 1914. Experiments on inheritance in parthenogenesis. Phil. Trans. Roy. Soc., London, 205 B : 421-489.

Baitsell, G. A., 1912. Experiments on the reproduction of the Hypotrichous Infusoria. I. Conjugation between closely related individuals of Stylonychia pustulata. Jour. Exper. Zool., 13:47-77.-Id., 1914. Experiments on the reproduction of the Hypotrichous Infusoria. II. A study of the so-called life cycle in Oxytricha fallax and Pleurotricha lanceolata. Jour. Exper. Zool., 16:211-235.-Balbiani, E. G., 1881-1882. Les Protozoaires. Journ. de Microg., T. 5 et 6 . (Cited from Bütschli, 1880.)-Barber, M. A., 1907. On heredity in certain micro-organisms. Kansas Univ. Sci. Bul., 4 (1); 1-47.-Bateson, W., 1914. The Address of the President of the British Association for the Advancement of Science. Science, 40:287-302, 319-333.-Blakeslee, A. F., 1904. Sexual reproduction in the Mucorineæ. Proc. Amer. Acad., 40:205-319.-Id., 1913. Conjugation in the heterogamic genus Zygorhynchus. Mycolog. Centralbl., 2:241-244.-Boti, K., 1907. Ueber die Fortpflanzung von Pelomyxa palustris, nebst Mitteilungen über ihren Bau. Arch. f. Protistenkunde, 8:120-158.-Bridges, C. B., 1916. Non-disjunction as proof of the chromosome theory of heredity. Genetics, 1:1-52, 107-163.-BrownSéquard, C. E., 1869. Nouvelles recherches sur l'Épilepsie due à certaines lésions de la moelle épinière et des nerfs rachidiens. Arch. Physiol. Norm. Path., 2:211-220, 422-437, 496-503.-Bütschli, O., 1875. Vorläufige Mittheilung einiger Resultate von Studien iber die Conjugation der Infusorien und die Zelltheilung. Zeitschr. f. wiss. Zool., 25: 426-441.-Id., 1880-82. Protozoa. Bronn's Klassen und Ordnungen des Thierreichs, Bd. 1. 2035 pp.

Calkins, G. N., 1902. Studies on the life-history of Protozoa. I. The life-cycle of Paramoecium caudatum. Arch. f. Entw.-Mech., 15:139186.-Id., 1902a. . Studies on the life-history of Protozoa. III. The six hundred and twentieth generation of Paramoecium caudatum. Biol. Bul., 3: 192-205.-Id., 1904. Studies on the life-history of Protozoa. IV. Death of the A series. Conclusions. Jour. Exp. Zool., 1:423-461.-Id., 1906. The Protozoan life cycle. Biol. Bul., 11:229-244.-Id., 1911. Regeneration and cell division in Uronychia. Jour. Exper. Zool., 10:95-116.-Id., 1915. Cycles and rhythms and the problem of "immortality" in Paramecium. Amer. Nat., 49:65-76.-Id., 1915a. Didinium nasutum. I. The life history. Jour. Exper. Zool., 19:225-241.-Id., 1916. General biology 
of the protozoan life cycle. Amer. Nat., 50:257-270.-Calkins, G. N., and Cull., S. W., 1907. The conjugation of Paramecium aurelia (caudatum). Arch. f. Protistenkunde, 10:375-415.-Calkins, G. N., and Gregory, L. H., 1913. Variations in the progeny of a single ex-conjugant of Paramecium caudatum. Jour. Exper. Zool., 15:467-525. -Carothers, E. E., 1917. The segregation and recombination of homologous chromosomes as found in two genera of Acrididae (Orthoptera). Jour. Morph., 28:445-520.-Castle, W. E., 1915. Mr. Muller on the constancy of Mendelian characters. Amer. Nat., 49:37-42.-Id., 1916. Further studies of piebald rats and selection, with observations on gametic coupling. Carnegie Institution Publ. 241, part III: 161-192.-Id., 1916 a. Genetics and eugenics. Cambridge. $353 \mathrm{pp} .-I d .$, 1917. The rôle of selection in evolution. Jour. Wash. Acad. Sci., 7:369-387.-Collin, B., 1909. La conjugaison d'Anoplophrya branchiarum (Stein) (A. circtlans Balbiani). Arch. d. Zool. Expér. et Gén., (5), 1:345-388.-Coulter, J. M., 1914. Evolution of sex in plants. $140 \mathrm{np}$. Chicago.-Cull, S. W., 1907. Rejuvenescence as a result of conjugation. Jour. Exper. Zool., 4: 85-89.

Dallinger, W. H., 1887. The President's address. Jour. Roy. Micr. Soc., 1:185-199.-Davenport, C. B., 1916. The form of evolutionary theory that modern genetical research seems to favor. Amer. Nat., 50:449-465.-Dobell, C. C., 1909. Chromidia and the binuclearity hypothesis. Quart. Jour. Micr. Sci., 53: 279-326.-Id., 1909 a. Physiological degeneration and death in Entamoeba ranarum. Quar. Jour. Micr. Sci., 53: 711-721. - Id., 1911. The principles of protistology. Arch. f. Protistenkunde, 23: 269-310.-Id., 1912. Some recent work on mutation in micro-organisms. Jour, of Genetics, 2: 201-220.-Id., 1914. A commentary on the genetics of the Ciliate Protozoa. Jour. of Genetics, 4: 131-190.-Doflein, F., 1911. Lehrbuch der Protozoenkunde. 1043 pp. Jena.

Enriques, $P$., 1903. Sulla cosi detta 'degenerazione senile' dei protozoa. Monitore Zool. Ital., 14:349-351.-Id., 1907. La coniugazione e il differenziamento sessuale negli Infusorî. Arch. f. Protistenkunde, 9:195296.-Id., 1908. Die Conjugation und sexuelle Differenzierung der Infusorien. Zweite Abhandlung. Wiederconjugante und Hemisexe bei Chilodon. Arch. f. Protistenkunde, 12:213-276.-Id., 1910. La coniugazione e il differenziamento sessuale negli infusorî. III. Azione dei sali sulle epidemie di coniugazione nel Cryptochilum nigricans. Memorie della R. Accademia delle Scienze dell'Istituto di Bologna, Serie VI, T. VI: 463-500,-Id., 1910 a. La coniugazione e il differenziamento sessuale negli infusorî. IV. Trattazione delle più importanti questioni. Mem. d. R. Accad. d. Sci. dell'Istituto di Bologna, Serie VI, T. VII: 161198.-Id., 1916. Duemila cinquecento geneazioni in un infusorio, senza coniugazione nè partenogenesi, nè depressioni. Rendiconto delle Sessioni della R. Accad. d. Scienze dell'Istituto di Bologna. 12 p. $-I d$., 1916 a. La coniugazione $\mathrm{e}$ il differenziamento sessuale negli infusorî. VI. Condizioni chi determinano la coniugazione ripetuta nel Chilodon uncinatus. Mem. d. R. Accad. d. Sci. dell'Istituto di Bologna, Serie VII, T. III : 49-54.-Erdmann, R., 1913. Experimentelle Ergebnisse über die Beziehung zwischen Fortpflanzung und Befruchtung bei Protozoen, besonders bei Amoeba diploidea. Arch. f. Protistenkunde, 29: 84-127.Erdmann, R., and Woodruff, L. L., 1916. The periodic reorganization process in Paramecium caudatum. Jour. Exper. Zool., 20: 59-97.-Ewing, 
H. E., 1916. Eighty-seven generations in a parthenogenetic pure line of Aphis avenae Fab. Biol. Bul., 31:53-112.

Fermor, $\boldsymbol{X}$., 1913. Die Bedeutung der Encystierung bei Stylonychia pustulata Ehrbg. Zool. Anzeiger, 42:380-384.-Fischer, E., 1907. Zur Physiologie der Aberrationen- und Varietäten-Bildung der Schmetterlinge. Arch. f. Rassen u. Gesellschaftsbiologie, 4: 761-793.

Geddes, P., and Thomson, J. A., 1880. The evolution of sex, 322 p. New York.

Hagedoorn, A. L., and A. C., 1914. Studies on variation and selection. Zeitschr. f. Ind. Abstammungslehre, 11:145-183.-Hance, R. T., 1917. Studies on a race of Paramecium possessing extra contractile vacuoles. I. An account of the morphology, physiology, genetics and cytology of this new race. Jour. Exper. Zool., 23: 287-327.-Hartmann, $M$., 1909. Autogamie bei Protisten und ihre Bedeutung für das $\mathrm{Be}-$ fruchtungsproblem. Arch. f. Protistenkunde, 14:264-334. $-I d$., 1914. Der Generationswechsel der Protisten und sein Zusammenhang mit dem Reduktions- und Befruchtungsproblem. Verhdlg. d. Deutsch. Zool. Ges., 24 Versaml.: 15-50.-Hertwig, R., 1889. Ueber die Conjugation der Infusorien. Abhldg. d. II. Cl. d. königl. bayr. Akad. d. Wiss., Bd. 17 (Abth. 1):151-233.-Id., 1914. Utber Parthenogenesis der Infusorien und die Depressionszustände der Protozoen. Biol. Centralbl., 34:557581.-Hutchison, R. H., 1913. Some specific differences among Protozoa with respect to their resistance to heat. Jour. Exper. Zool., 15: 131144.-Id., 1915. The effects of certain salts, and of adaptation to high temperatures, on the heat resistance of Paramecium caudatum. Jour. Exper. Zool., 19:211-224.-Hyde, R. R., 1916. Two new members of a sex-linked multiple (sextuple) allelomorph system. Genetics, 1:535-580.

Jennings, H. S., 1906. Behavior of the Lower Organisms. 366 pp. New York.-Id., 1908. Heredity, Variation and Evolution in Protozoa. I. The fate of new structural characters in Paramecium, in connection with the problem of the inheritance of acquired characters in unicellular organisms. Jour. Exper. Zool., 5:577-632.-Id., 1908 a. Heredity, Variation and Evolution in Protozoa. II. Heredity and variation of size and form in Paramecium, with studies of growth, environmental action and selection. Proc. Amer. Philos. Soc., 47:393-546.-Id., 1909. Heredity and variation in the simplest organisms. Amer. Nat., 43:321-337.$I d .$, 1910. What conditions induce conjugation in Paramecium? Jour. Exper. Zool., 9: 279-298.-Id., 1911. Assortative mating, variability and inheritance of size, in the conjugation of Paramecium. Jour. Exper. Zool., 11:1-134.-Id., 1912. Age, death and conjugation in the light of work on lower organisms. Pop. Sci. Monthly, 80:563-577. $-I d$., 1913. The effect of conjugation in Paramecium. Jour. Exper. Zool., 14: 279391.-Id., 1916. Heredity, variation and the results of selection in the uniparental reproduction of Difflugia corona. Genetics, 1: 407-534.- Id., 1917. Observed changes in hereditary characters in relation to evolution. Jour. Wash. Acad. Sci., 7: 281-301.-Jennings, H. S., and Hargitt, G. T., 1910. Characteristics of the diverse races of Paramecium. Jour. Morph., 21:495-561.-Jenning8, H. S., and Lashley, K. S., 1913. Biparental inheritance and the question of sexuality in Paramecium. Jour. Exper. Zool., 14:393-466.-Id., 1913 a. Biparental inheritance of 
size in Paramecium. Jour. Exper. Zool., 15:193-199.-Johannsen, $W$., 1903. Ueber Erblichkeit in Populationen und in reinen Linien. $v+68$ pp. Jena.-Id., 1913. Elemente der exakten Erblichkeitslehre. Zweite Ausgabe. xi +723 pp. Jena.-Jollos, V., 1913. Über die Bedeutung der Conjugation bei Infusorien. Arch. f. Protistenkunde, 30:328-334.Id., 1913 a. Experimentelle Untersuchungen an Infusorien. Biol. Centralbl., 33:222-236.-Id., 1914. Variabilität und Vererbung bei Mikroorganismen. Zeitschr. f. Ind. Abstammungslehre, 12: 14-35.-Id., 1916. Die Fortpflanzung der Infusorien und die potentielle Unsterblichkeit der Einzelligen. Biol. Centralbl., 36:497-514.

Kammerer, $P$., 1913. Vererbung erzwungener Farbenveränderungen. IV. Mitteilung. Das Farbkleid des Feuersalamanders (Salamandra maculosa Laurenti) in seiner Abhängigkeit von der Umwelt. Arch. f. Entw. Mech., 36: 1-193.

Lashley, K. S., 1915. Inheritance in the Asexual Reproduction of Hydra. Jour. Exper. Zool., 19:157-210.-Lotsy, J. P., 1916. Qu'est-ce qu'une espèce? Arch. Néerlandaises d. Sci. Exactes et Nat., Sér. III B, T. III: $57-110 .-I d ., 1916 a$. Sur les rapports entre des problèmes sociaux et quelques résultats de la théorie moderne de l'hérédité. Arch. Néerlandaises d. Sci. Exactes et Nat., Série III B, T. III: 151-175.

MacDowell, E. C., 1915. Bristle inheritance in Drosophila. Jour. Exper. Zool., 19:61-97.-Id., 1916. Piebald rats and multiple factors. Amer. Nat., 50: 719-742.-Massini, R., 1907. Über einen in biologischer Beziehung interessanten Kolistamm (Bacterium coli mutabile). Arch. f. Hygiene, 61: 250-292.-Mast, S. O., 1917. Conjugation and encystment in Didinium nasutum with especial reference to their significance. Jour. Exper. Zool., 23:335-359.-Maupas, E., 1888. Recherches expérimentales sur la multiplication des infusoires ciliés. Arch. d. Zool. Exp. et Gén. (2), 6:165-277.-Id., 1889. La rajeunissement karyogamique chez les ciliés. Arch. d. Zool. Exp. et Gén. (2), 7:149-517.-Metcalf, M. M., 1909. Opalina. Its anatomy and reproduction, with a description of infection experiments and a chronological review of the literature. Arch. f. Protistenkunde, 13; 195-375.-Middleton, A. R., 1915. Heritable variations and the results of selection in the fission rate of Stylonychia pustulata. Jour. Exper. Zool., 19:451-503.-Id., 1918. Inherited effects of diversity of temperature in Stylonychia pustulata. Genetics, 3:534-572.-Minchin, E. A., 1912. An introduction to the study of the Protozoa. $520 \mathrm{pp}$. London.-Morgan T. H., 1916. A critique of the theory of evolution. $197 \mathrm{pp}$. Princeton.-Id., 1917. The theory of the gene. Amer. Nat., 51:513-544.-Id., 1917 $a$. An examination of the so-called process of contamination of genes. Anat. Record, 11:503-504.-Mulsow, $K ., 1911$. Ueber Fortpflanzungserscheinungen bei Monocystis rostrata, n. sp. Arch. f. Protistenkunde, 22:20-55.

Nenoman. $\boldsymbol{H} . \boldsymbol{H}$. 1917. The biology of twins. $185 \mathrm{pp}$. Chicago.

Osborn, H. F., 1917. The origin and evolution of life. 322 pp., New York.

Pearl, R., 1915. Seventeen years' selection of a character showing sex-linked Mendelian inheritance. Amer. Nat., 49:595-608.-Id., 1916. Fecundity in the domestic fowl and the selection problem. Amer. Nat., 
50:89-105.-Id., 1917. The selection problem. Amer. Nat., 51:65-91.Id., 1917 a. The experimental modification of germ cells. Jour. Exper. Zool., 22: 125-186, 241-310.-Popoff, M., 1908. Die Gametenbildung und die.Conjugation von Carchesium polypinum L. Zeitschr. f. wiss. Zool., 89:478-524.-Powers, J. H., and Mitchell, C. W., 1910. A new species of Paramecium (P. multimicronucleata) experimentally determined. Biol. Bul., 19:324-332.-Prandtl, H., 1906. Die Konjugation von Didinium nasutum O. F. M. Arch. f. Protistenkunde, 7:229-258.-Pringsheim, H., 1910. Die Variabilität niederer Organismen. $216 \mathrm{pp}$. Berlin.-Prowazek, S., 1904. Die Entwicklung von Herpetomonas. Arb. a. d. kais. Gesundheitsamte, 20.

Quehl, A., 1906. Untersuchungen über die Myxobakterien. Bakt. Centralbl., Abt. 2, 16:9-34.

Radl, Em., 1909-1913. Geschichte der Biologischen Theorien. 2 Bde. $351+604$ p. Leipzig.-Reeves, E. M., 1916. The inheritance of extra bristles in Drosophila melanogaster Meig. Univ. of Calif. Publ. in Zoology, 13:495-515.-Robertson, W. R. B., 1908. The chromosome complex of Syrbula admirabilis. Kansas University Science Bul., 4:275305.

Safir, S. R., 1916. Buff, a new allelomorph of white eye color in Drosophila. Genetics, 1:584-590.-Schaudinn, F., 1904. Generationsund Wirtswechsel bei Trypanosoma und Spirochaete. Arb. a. d. kais. Gesundheitsamte, 20.-Sharp, R. G., 1914. Diplodinium ecaudatum, with an account of its neuromotor apparatus. Univ. of Calif. Publ. in Zoology, 13: 43-122.—Standfuss, M., 1906. Die Resultate dreissigjähriger Experimente mit Bezug auf Artenbildung und Umgestaltung in der Tierwelt. Verhdlg. d. Schweiz. Naturf. Ges. Luzern (1905). - Stockard, C. $R$., 1913. The effect on the offspring of intoxicating the male parent and transmission of the defects to subsequent generations. Amer. Nat., 47:641-682.-Stocking, R. J., 1915. Variation and inheritance in abnormalities occurring after conjugation in Paramecium caudatum. Jour. Exper. Zool., 19:387-449.-Sturtevant, A. H., 1917. An analysis of the effect of selection on bristle number in a mutant race of Drosophila. Anat. Record, 11:504.

Toenniessen, E., 1915. Ûber Vererbung und Variabilität bei Bakterien. Biol. Centralbl., 35:281-330.-Tower, W. L., 1906. An investigation of evolution in chrysomelid beetles of the genus Leptinotarsa. Carnegie Inst. Publ. 48:320 p.

Verworn, M., 1888. Biologische Protisten-Studien. Zeitschr. f. wiss. Zool., 46:455-470.-Vries, $H$. de, 1901. Die Mutationstheorie. 2 vols.: $648+752$ pp. Leipzig.-Id., 1916. Die Grundlinien der Mutationstheorie. Die Naturwissenschaften, Hft. 40:17 p.

Wallengren, $H .$, 1899. Ueber die totale Konjugation bei Vorticellina. Biol. Centralbl., 19: 153-161.-Id., 1901. Zur Kenntniss des Neubildungsund Resorptionsprocesses bei der Theilung der hypotrichen Infusorien. Zool. Jahrb., 15: 1-58.-Walton, L. B., 1915. Variability and amphimixis. Amer. Nat., 49:649-687.-Watters, F. A., 1912. Size relationships between conjugants and non-conjugants in Blepharisma undulans. Biol. Bul., 23: 195-212.-Wenrich, D. H., 1916. The spermatogenesis of 
Phrynotettix magnus with special reference to synapsis and the individuality of the chromosomes. Bul. Mus. Comp. Zool. Harvard, 60:57133.-Wilson, E. B., 1910. The chromosomes in relation to the determination of sex. Science Progress, No. 16:570-592.-Id., 1911. Studies on chromosomes. VII. A review of the chromosomes of Nezara; with some more general considerations. Jour. Morph., 22:71-110.-Wolf, $F$., 1909. Über Modifikationen und experimentell ausgelöste Mutationen von Bacillus prodigiosus und anderen Schizophyten. Zeitschr. f. ind. Abst. u. Vererb., 2:90-132.-Woodruff, L. L., 1914. So-called conjugating and non-conjugating races of Paramecium. Jour. Exp. Zool., 16: 237-240.-Id., 1915. The problem of rejuvenescence in Protozoa. Biochem. Bul., 4:371-378. - Id., 1917. Rhythms and Endomixis in Various Races of Paramecium aurelia. Biol. Bul., 33:51-56.-Id., 1917 a. The influence of general environmental conditions on the periodicity of endomixis in Paramecium aurelia. Biol. Bul., 33:437-462.-Woodruff, L. L., and Erdmann, R., 1914. A normal periodic reorganization process without cell fusion in Paramecium. Jour. Exper. Zool., 17:425-518.

Young, R. T., 1917. Experimental induction of endomixis in Paramecium aurelia. Jour. Exper. Zool., 24:35-53.

Zeleny, C., and Mattoon, E. W., 1915. The effect of selection on the "bar-eyes" mutant of Drosophila. Jour. Exper. Zool., 15:515-529.$Z$ weibaum, J., 1912. La conjugaison et la différenciation sexuelle chez les infusoires (Enriques et Zweibaum). V. Les conditions nécessaires et suffisantes pour la conjugaison du Paramecium caudatum. Arch. f. Protistenkunde, 26:275-393. 



\section{INDEX}

Abnormalities, 150, 159

Acclimatization, 97

Ackert, 63

Acquired characters, 44

Agar, 64

Alcohol, 203

Amœba, 15, 39

Amphibia, 200

Anabolism, 115

Anoplophrya, 180, 192

Arcella, 56, 77, 82

Arsenic, 101

Assortative mating, 191

Aster, 117

A utogamy, 134, 185

Bacillus coli, 88

Bacillus prodigiosus, 63,88

Bacteria, 15, 50, 58, 63, 86-96

Balbiani, 24

Barber, 63, 87

Bateson, 68, 215

Beans, 64

Biparental inheritance, 151, 152, 168, 170-190

Biparental reproduction, 21, 219

Blakeslee, 123

Blepharisma, 192

Bott, 183

Bridges, 208, 210

Brown-Séquard, 203

Bütschli, 24

Butterflies, 202

Cænomorpha, 16

Calkins, 24, 29, 32, 120, 141, 151

Calkins and Cull, 181

Calkins and Gregory, 142

Carchesium, 180

Castle, 203, 207

Catabolism, 116

Centropyxis, 56, 77

Centrosome, 116

Chemical agents, 88-96, 102, 203
Chilodon, 128, 180

Chlamydomonas, 155-157

Chromidium, 83

Chromosomes, 108, 115, 116, 138, 171-190

Climacostomum, 150

Collin, 126, 180, 192

Colored bacteria, 88

Colpoda, 165

Constancy of stocks, 62, 65, 67, 86

Corrosive sublimate, 89

Coulter, 126

Conditions of conjugation, 164

Conjugation, 22, 25, 36, 102, 106169

Continuity of life, 19, 21, 30, 35

Corpse, 35

Correlation, 73

Crustacea, 64

Cryptochilum, 165

Cull, 120

Dallinger, 98

Dallingeria, 98

Davenport, 68, 215

Death, 19, 28, 39

Democracy, 222

Depression, 148

Dexter, 208

Didinium, 16, 32, 133, 142, 150, 177,195

Difflugia, 40, 45, 50-55, 68, 70-76

Diplodinium, 17

Dobell, 93, 95, 96, 106, 153

Doflein, 119

Draba, 59

Drosophilia, 205, 207, 208, 223

Endomixis, 31

Enriques, 29, 33, 128, 165, 180

Environmental modifications, 85103, 198, 218

Epistylis, 121

Erdmann, 166 
Euplotes, 146

Evolution, 38, 65-73, 83, 213, 223

Ewing, 64

Exchange of nuclei, 25, 36, 110

Exconjugant, 112

Eye color, 208-211

Female, 115, 116, 133

Fermor, 32

Fertilization, 36, 106

Fischer, 202

Fission rate, 24, 81, 141-147, 154, 158,161

Flagellate, 134

Folliculina, 16

Fowl, 203

Geddes and Thompson, 115

Genotype, 65, 86

Glaucoma, 29, 33

Gradations, 76, 209, 213-218

Gregarinidae, 175

Guinea pig, 203

Hance, 58

Hartmann, 137

Hegner, 56, 77, 82

Heredity, 37-49, 67-84, 152-157, $170-190$

Heritable variations, $76,81,84$, 212

Herpetomonas, 183

Hertwig, R., 24, 142, 144-148

Hutchison, 57

Hydra, 64.

Hypotricha, 42

Immortality of unicellular organisms, $20,30,35$

Infusoria, 16, 17, 24

Inheritance, 37-49, 67-84, 152-157, 170-190

Inheritance of acquired characters, $44,48,85-105,199,218$

Isolation of bacterium, 87

Johannsen, 64, 65

Jollos, 33, 34, 57, 63, 95, 101

Jordan, 59, 66

Kammerer, 200, 202

Kinetic, 116
Kinetonucleus, 96, 97, 116

Kinetoplasm, 116

Lacrymaria, 16

Lashley, 64, 124

Leucophrys, 28, 146, 150

Lotsy, 59

MacDowell, 207, 208

Macronucleus, 25, 31, 35, 44, 103, 110

Male, 115, 116, 133

Massini, 92

Mast, 142, 195

Maturation, 172-186

Maupas, 24, 28, 30, 64, 114, 121, 122 $131,132,142,145,146,147,150$

Mendelian inheritance, 65, 187-190

Metcalf, 181

Micronucleus, 25, 31, 44, 58, 103, 110

Middleton, 80, 100

Migratory nucleus, 110, 132

Minchin, 126

Modification, 103

Modification of inherited characters by external agents, 85-103, 198, 218

Modifying factors, 207, 210

Monas, 98

Monocystis, 116

Monstrosities, 150, 159

Morgan, 205, 207, 208

Mould, 123, 136

Mucor, 123

Muller, 208

Mulsow, 175

Multiple allelomorphs, 209

Mutation, 67, 68, 102, 204-211

Mutilations, inheritance of, 45

Myxobacteria, 93

Myxococcus, 93

Newman, 60

Non-disjunction, 210

Nucleo-plasmic relation, 148

Nucleus, 24, 25, 82

QEnothera, 205

Onychodromus, 28, 146

Opalina, 181

Opercularia, 128, 180

Osborn, 215

Oxytricha, 28 
Palæontology, 69, 215

Paramecium, 22, 25, 29, 31, 34, 46, $50,56,58,63,68,97,110,119$, $130,133,142,143,154,158,165$, 181, 191

Parthenogenesis, 64

Pearl, 68, 203

Pelomyxa, 183

Plant lice, 64

Pneumonia bacillus, 90

Popoff, 180

Potassium bichromate, effects of, 89

Potato beetle, 202

Powers and Mitchell, 58

Prandtl, 133, 177

Primary hereditary characters, 170, 185-187, 222

Pringsheim, 95

Protozoa, 15

Prowazek, 183

Pure line, 65

Purpose, 106

Quehl, 93

Radl, 13

Rate of reproduction, $24,81,141-$ $147,154,158,161$

Reduction, 172, 175-186

Reeves, 207

Rejuvenescence, 23, 26, 36, 114, $141-147,150,162,220$

Reproduction, rate of, 24, 81, 141$147,154,158,161$

Reserve nucleus, 25

Resistance, 57, 101

Root, 56, 77

Saltation, 205

Schaudinn, 183

Secondary hereditary characters, 185

Selection, 60-73, 81

Senility, 30

Sex, 109, 114-140, 168, 219

Size of nuclei, 82

Spirogyra, 135

Spirostomum, 150
Stand fuss, 202

Stentor, 16

Stephanosphæra, 126

Stockard, 203

Structure of Protozoa, 15

Sturtevant, 207, 208

Stylonychia, 16, 28, 32, 43, 81, 100, 145,150

Temperature, acclimatization to, 98-100

Tetramitus, 98

Toenniessen, 90

Tower, 202

Trichomastix, 134

Trophic, 116

Trophonucleus, 116

Trophoplasm, 116

Trypanosoma, 96, 183

Twins, 60

Unicellular organisms, 15, 16

Uniparental inheritance, 38-84

Uniparental reproduction, 21

Unit factor, 208

Uroleptus, 32, 142, 144, 149, 151, 163

Vacuoles, contractile, 58

Variation, 50, 61, 66, 67-72, 76, 193

Vitality, 24, 141-147, 161, 196

Vries, de, 205, 212

Vorticella 16, 120, 127

Wallengren, 121

Walton, 195

Watters, 192

Wolf, 63, 88, 93

Woodruff, 29, 30, 33, 149, 167

Woodruff and Erdmann, 30, 32, 34, 103,145

Yeasts, 63

Young, 33

Zeleny and Mattoon, 207

Zweibaum, 165

Zygorhynchus, 136

Zygospore, 123 

•

. 
- 

441 


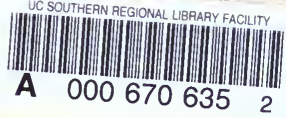


